\title{
Coalfields of New Mexico: Geology and Resources
}

\section{U.S. GEOLOGICAL SURVEY BULLETIN 1972}

Prepared in cooperation with the New Mexico Bureau of Mines and Mineral Resources

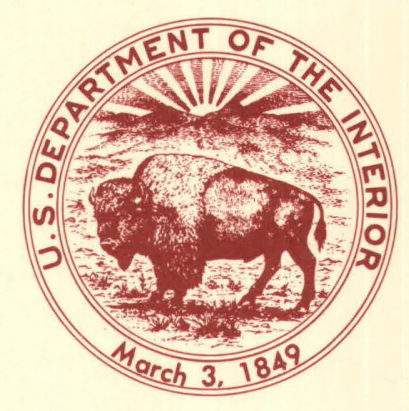




\section{AVAILABILITY OF BOOKS AND MAPS OF THE U.S. GEOLOGICAL SURVEY}

Instructions on ordering publications of the U.S. Geological Survey, along with the last offerings, are given in the current-year issues of the monthly catalog "New Publications of the U.S. Geological Survey." Prices of available U.S. Geological Survey publications released prior to the current year are listed in the most recent annual "Price and Availability List." Publications that are listed in various U.S. Geological Survey catalogs (see back inside cover) but not listed in the most recent annual "Price and Availability List" are no longer available.

Prices of reports released to the open files are given in the listing "U.S. Geological Survey Open-File Reports," updated monthly, which is for sale in microfiche from the U.S. Geological Survey, Books and Open-File Reports Section, Federal Center, Box 25425, Denver, CO 80225. Reports released through the NTIS may be obtained by writing to the National Technical Information Service, U.S. Department of Commerce, Springfield, VA 22161; please include NTIS report number with inquiry.

Order U.S. Geological Survey publications by mail or over the counter from the offices given below.

\section{BY MAIL}

\section{Books}

Professional Papers, Bulletins, Water-Supply Papers, Techniques of Water-Resources Investigations, Circulars, publications of general interest (such as leaflets, pamphlets, booklets), single copies of periodicals (Earthquakes \& Volcanoes, Preliminary Determination of Epicenters), and some miscellaneous reports, including some of the foregoing series that have gone out of print at the Superintendent of Documents, are obtainable by mail from

U.S. Geological Survey, Books and Open-File Reports Section Federal Center, Box 25425

Denver, CO 80225

Subscriptions to periodicals (Earthquakes \& Volcanoes and Preliminary Determination of Epicenters) can be obtained ONLY from

\section{Superintendent of Documents \\ U.S. Government Printing Office Washington, DC 20402}

(Check or money order must be payable to Superintendent of Documents.)

\section{Maps}

For maps, address mail orders to

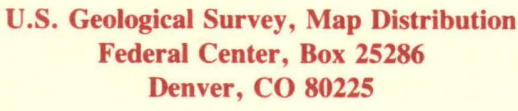

Residents of Alaska may order maps from

\author{
U.S. Geological Survey, Map Distribution \\ New Federal Building - Box 12 \\ 101 Twelfth Ave., Fairbanks, AK 99701
}

\section{OVER THE COUNTER}

\section{Books}

Books of the U.S. Geological Survey are available over the counter at the following U.S. Geological Survey offices, all of which are authorized agents of the Superintendent of Documents:

- ANCHORAGE, Alaska-4230 University Dr., Rm. 101

- ANCHORAGE, Alaska-605 West 4th Ave., Rm. G-84

- DENVER, Colorado-Federal Bldg., Rm. 169, 1961 Stout St.

- LOS ANGELES, California-Federal Bldg., Rm. 7638 . 300 North Los Angeles St.

- MENLO PARK, California-Bldg. 3, Rm. 3128, 345 Middlefield Rd.

- RESTON, Virginia-National Center, Rm. IC402, 12201 Sunrise Valley Dr.

- SALT LAKE CITY, Utah-Federal Bldg., Rm. 8105, 125 South State St.

- SAN FRANCISCO, California-Customhouse, Rm. 504 , 555 Battery St.

- SPOKANE, Washington-U.S. Courthouse, Rm. 678, West 920 Riverside Ave.

- WASHINGTON, DC-U.S. Department of the Interior Bldg., Rm. 2650, 1849 C St., NW.

\section{Maps}

Maps may be purchased over the counter at the U.S Geological Survey offices where books are sold (all addresses in above list) and at the following U.S. Geological Survey offices:

- ROLLA, Missouri-1400 Independence Rd.

- FAIRBANKS, Alaska-New Federal Bldg., 101 Twelfth Ave. 


\section{Coalfields of New Mexico: Geology and Resources}

Edited by CAROL L. MOLNIA, DANIEL A. JOBIN, and JOSEPH T. O'CONNOR

U.S. Geological Survey, and

FRANK E. KOTTLOWSKI

New Mexico Bureau of Mines and Mineral Resources

Prepared in cooperation with the New Mexico

Bureau of Mines and Mineral Resources

Thickness and distribution of coal beds, estimates of the resources, and descriptions

of methods used for calculating resources 


\title{
U.S. DEPARTMENT OF THE INTERIOR
}

MANUEL LUJAN, JR., Secretary

\author{
U.S. GEOLOGICAL SURVEY
}

Dallas L. Peck, Director

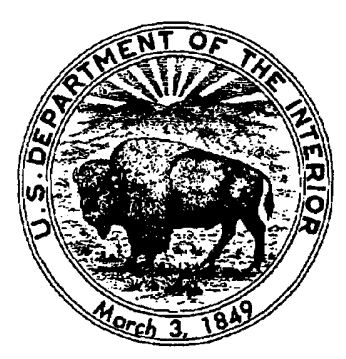

Any use of trade, product, or firm names in this publication is for descriptive purposes only and does not imply endorsement by the U.S. Government.

UNITED STATES GOVERNMENT PRINTING OFFICE: 1991

For sale by the

Books and Open-File Reports Section

U.S. Geological Survey

Federal Center

Box 25425

Denver, CO 80225

\section{Library of Congress Cataloging-in-Publication Data}

Coalfields of New Mexico: geology and resources / edited by Carol L. Molnia ... [et al.].

p. cm. - (U.S. Geological Survey bulletin ; 1972)

"Prepared in cooperation with the New Mexico Bureau of Mines and Mineral Resources."

Includes bibliographical references.

Supt. of Docs. no.: I 19.3:1972

1. Coal-New Mexico. 2. Coal-Geology—New Mexico. I. Molnia, Carol L. II. New Mexico. Bureau of Mines and Mineral Resources. III. Series.

QE75.B9 no. 1972

[TN805.N6]

$557.3 \mathrm{~s}-\mathrm{dc} 20$

[553.2' $\left.4^{\prime} 09789\right]$

$91-11586$ 


\section{PREFACE}

Calculation of the coal resources of New Mexico is an important part of the evaluation of State, Federal, and privately owned energy potential. As such, it bears directly on the economic growth and development potential of many areas within the State. To be of most use, coal resource data must accurately reflect the quantity of coal present and its accessibility to development. In this report, the State Geologist of New Mexico and the U.S. Geological Survey describe the primary geologic factors that influence the quantity and accessibility of the coal resources of New Mexico. The report gives greatest emphasis to the San Juan basin, an area that has provided much of the publicly available data and that retains a large portion of present coal development interest.

The chapters that follow include brief geological descriptions of New Mexico's coalfields, current estimates of the amount of coal resources present in each of them, analyses of the probable accuracy of such estimates, determinations of thickness of known and projected coal beds or coal zones, and discussions of the geologic factors that affect the coal resources.

The authors have assessed the current state of knowledge of the coal resources of New Mexico, pointing out inadequacies in the data base and understanding of the resources. Though our primary data source has been the National Coal Resources Data System of the U.S. Geological Survey, we have also used data from other sources to make this report as complete as possible.

This report is a product of many people's efforts. Besides the authors and editors, other major contributors from the U.S. Geological Survey include Dorsey Blake, for guidance in computer operations; Courteney Williamson, for data entry, validation, and graphics generation; Noreen $\mathrm{H}$. Rega, for data base development, validation, and resource calculation; Mary E. Lemaster and Patricia T. Kerr, for resource calculation and data validation; and Andre D. Bush and Paula M. Washington, for digitizing and boundary data processing. Margaret F. Johnson and Brian R. Schachte helped with new record processing and provided graphics display support; Frank E. Andreani helped with data formatting, processing, and updating.

Assistance was also given by Laura N.R. Roberts, in computer software use; Jean K. Dillinger, Annabel B. Olson, and James E. Fassett, in San Juan basin geology; and Margaret S. Ellis, in data validation. Many of the personnel above helped with drafting, as did Arthur C. Clark. J. Daniel Cathcart photographed the illustrations. The assistance of the secretarial staff in the preparation of the text is also appreciated.

The authors of chapter $\mathrm{E}$ were aided by contributions from other members of the New Mexico Bureau of Mines and Mineral Resources, particularly the drafting of Michael Wooldridge and the useful suggestions of Orin J. Anderson and JoAnn C. Osburn. 



\section{CONTENTS}

[Letters designate the chapters]

(A) Geologic framework and major coal-bearing formations of the San Juan basin, by Morris W. Green, James W. Mytton, Dorothy T. Sandberg, and

Nancy K. Gardner 1

(B) Coal resources of the San Juan basin, by Laura R.H. Biewick, Antoinette L. Medlin, Judy F. Hunter, and Kathleen K. Krohn 15

(C) Coal resource estimation in the San Juan basin using geostatistical methods, by Lewis W. Boger, Jr., and Antoinette L. Medlin 35

(D) Geology and coal resources of the Raton coalfield, by Charles L. Pillmore 45

(E) Geology and coal resources of New Mexico's small coalfields, by Frank W. Campbell, Gretchen K. Hoffman, Frank E. Kottlowski, and Brian W. Arkell

69 


\section{METRIC CONVERSION FACTORS}

Data in this volume are reported in customary inch-pound units because the metric system is not currently in use by the coal industry of the United States. Readers wishing to convert measurements to the International System of units (SI) may use the following factors:

\begin{tabular}{ll}
\hline \multicolumn{1}{c}{ U.S. customary unit } & \multicolumn{1}{c}{ SI conversion } \\
\hline Acre & $=4,046.87$ square meters \\
Acre-foot & $=1,233.49$ cubic meters \\
British thermal unit (Btu) & $=1,055.056$ joules \\
Btu/lb & $=2,326$ joules per kilogram \\
Foot (ft) & $=0.3048$ meters \\
Inch (in.) & $=0.0254$ meters \\
Mile (mi) & $=1.609$ kilometers \\
Pound (lb) & $=0.4536$ kilograms \\
Short ton (ton) & $=0.9072$ metric tons \\
Square miles $\left(\mathrm{mi}^{2}\right)$ & $=2.59$ square kilometers \\
Ton/acre-foot & $=0.7355$ kilograms per cubic meter \\
\hline
\end{tabular}


Chapter A

\section{Geologic Framework and Major Coal-Bearing Formations of the San Juan Basin}

BY MORRIS W. GREEN, JAMES W. MYTTON, DOROTHY T. SANDBERG, and NANCY K. GARDNER

Upper Cretaceous strata preserved in a Laramide-age structural basin host New Mexico's largest coal deposits 


\title{
CONTENTS
}

\author{
Abstract 3 \\ Introduction $\mathbf{3}$ \\ Location and geography 3 \\ Geologic setting 4 \\ Tertiary tectonic history and structure 4 \\ Paleozoic and Mesozoic tectonics and sedimentology 4 \\ Coal geology 8 \\ References cited \\ 11

\section{FIGURES}

A1. Generalized geologic map of the San Juan basin and adjacent region $\mathbf{5}$

A2. Stratigraphic correlation chart of the San Juan basin 6

A3. Diagrammatic cross section of the San Juan basin 7

A4. Structure contour perspective on the base of the Dakota Sandstone, San Juan basin 8

A5. Time-stratigraphic geologic section of Cretaceous rocks, San Juan basin 9

A6. Fence diagrams showing stratigraphic rise of the Pictured Cliffs Sandstone across the San Juan basin 10

A7. Correlation of well logs showing lenticular nature of coal within the Fruitland Formation 12

A8. Map of Upper Cretaceous formations, fields, areas, and mines containing strippable low-sulfur coal in the San Juan basin 


\title{
Geologic Framework and Major Coal-Bearing Formations of the San Juan Basin
}

\author{
By Morris W. Green, James W. Mytton, Dorothy T. Sandberg, and Nancy K. Gardner
}

\begin{abstract}
The San Juan basin, New Mexico's leading coal producing area, is a structural basin formed during the Laramide orogeny ( 80 to 40 million years ago). The basin lies primarily in northwest New Mexico but extends into southwest Colorado and northeast Arizona and covers an area of about 26,000 square miles on the southeast margin of the Colorado Plateau physiographic province. Approximately 12,000 feet of Paleozoic and Mesozoic rocks are preserved in the basin. Cretaceous rocks, covered by Tertiary rocks in the central part of the basin, account for approximately one-half of the total thickness. The sedimentary interval in the basin locally hosts some of the Nation's most important deposits of coal, uranium, oil, and gas. Coal deposits occur in deltaic and coastal plain lithofacies throughout the Upper Cretaceous interval over relatively wide areas of the basin. The geographic location and stratigraphic position of coal beds of Cretaceous age in the San Juan basin were determined by the location of ancient shorelines along the western border of an epicontinental sea during Late Cretaceous time. Coal deposits originated from thick accumulations of organic matter (peat) in paludal environments on deltaic and lower coastal plains landward of shorelines. These environments formed as a result of periodic transgression and regression of the sea. The coal beds tend generally to be elongate to the northwest, parallel to the shoreline of the sea, which retreated progressively farther northeast with each successive major transgressive-regressive cycle of Late Cretaceous time. By the end of the Cretaceous Period, the sea had withdrawn from the region completely.

The major Cretaceous coal-bearing units, in ascending order, are the Crevasse Canyon and Menefee Formations of the Mesaverde Group and the Fruitland Formation, which contains by far the largest coal resources in the San Juan basin. The thickness and distribution of coal in the Fruitland are inversely related to increase in thickness and stratigraphic rise of the underlying Pictured Cliffs Sandstone, especially where
\end{abstract}

Manuscript approved for publication January 10, 1991. the Fruitland Formation intertongues with the upper part of the Pictured Cliffs. Typically, the coal beds are highly lenticular and difficult to correlate between drill holes; they can be correlated with certainty only where the holes are closely spaced. For these reasons, coal resource calculations can only be made for coal zones, rather than individual beds. Fields or areas in the San Juan basin where the Fruitland Formation is the current or potential coal producer are the Navajo field and Bisti and Star Lake areas. The remaining fields and areas are current or potential producers of coal from the Mesaverde Group and its equivalents, the most notable being the Gallup field.

\section{INTRODUCTION}

The San Juan basin of northwest New Mexico is the State's leading producer of coal. The basin's coal and its other energy mineral deposits-uranium, oil, and gasmake it one of the Nation's most important energy-rich sedimentary basins. The coal deposits, restricted in occurrence to the upper part of the basin sedimentary sequence in formations of Cretaceous age, supply fuel for the electric power industry throughout the southwestern United States.

This report contributes to the joint effort by the U.S. Geological Survey and the New Mexico Bureau of Mines and Mineral Resources to provide a current overview of the geology and coal resources for the State of New Mexico.

\section{LOCATION AND GEOGRAPHY}

The San Juan basin is located primarily in northwest New Mexico. The northern and western areas of the basin extend, respectively, into neighboring southwest Colorado and northeast Arizona. The basin has dimensions of approximately 200 miles north to south and 130 miles east to west. The basin is irregularly circular and covers an area 
of about 26,000 square miles on the southeast margin of the Colorado Plateau physiographic province.

The basin is bounded on all sides by mountain ranges: the Zuni Mountains on the south, the Nacimiento Mountains on the east, the La Plata and San Juan Mountains on the north in Colorado, and the Defiance uplift and the Chuska and Carizzo Mountains on the west near the Arizona-New Mexico border. The boundary of the basin is generally placed where the contact between Cretaceous rocks and underlying older rocks crops out near the flanks of the surrounding mountain ranges (fig. A1).

\section{GEOLOGIC SETTING}

The San Juan basin, like other major present-day sedimentary basins in the Western Interior of the U.S., formed during the Laramide orogeny, which spanned the time interval between the Late Cretaceous and the middle Tertiary ( 80 to 40 million years ago). Approximately 12,000 feet of rocks ranging in age from Cambrian to Cretaceous are preserved in the basin (fig. A2). Only Ordovician and Silurian age rocks are absent in the sequence. Cretaceous rocks, covered by Tertiary rocks in the central part of the basin, account for approximately one-half of the total thickness of the Paleozoic and Mesozoic sequence.

The sedimentary interval in the basin locally hosts some of the Nation's most important deposits of coal, uranium, oil, and gas. Large uranium deposits occur in fluviolacustrine sandstone lithofacies of the Upper Jurassic Morrison Formation in the southern part of the basin. Oil, gas, and coal deposits occur in marine and coastal plain lithofacies throughout the Upper Cretaceous interval over much of the basin.

\section{TERTIARY TECTONIC HISTORY AND STRUCTURE}

The San Juan basin is a structural basin bounded by uplifts which formed as a result of Laramide crustal movements. The basin is asymmetrical north to south, having its deeper axial part in the north-northeastern portion (fig. A3). Because the basal Upper Cretaceous Dakota Sandstone is uniformly distributed and widespread in the basin, it is frequently used in depicting Laramide deformation (fig. A4).

The San Juan basin apparently formed on an internally stable, subsiding crustal block surrounded by crustal blocks which were uplifted to form the cores of adjacent mountain ranges. Differential subsidence and uplift of crustal blocks in the San Juan basin (as well as elsewhere in the Western Interior) apparently resulted from regional tectonic compression (crustal shortening) attendant to active western plate margin tectonism associated with westward motion of the North American plate during the time of the Laramide orogeny.

Locally, pre-Tertiary sedimentary rocks are sharply upturned against the flanks of surrounding uplifts. Structurally deformed sedimentary units strike parallel to the flanks of marginal uplifts and dip steeply basinwardlocally exceeding $80^{\circ}$-across the hinge lines separating the uplifted blocks and the subsided block. Basinward, the dip angle of the units abruptly decreases to $3^{\circ}-5^{\circ}$. Local structural features, generally confined to the hinge areas of the basin, include normal faults and fault zones, small anticlines and synclines, structural platforms, and monoclines. With the exception of faults and fault zones, which trend normal to the flanks of uplifts, local structural features trend parallel to the basin margin. Relative to basin margin areas, the basin interior is essentially structureless, indicating relative stability of the crustal block flooring the basin.

\section{PALEOZOIC AND MESOZOIC TECTONICS AND SEDIMENTOLOGY}

Paleozoic and Mesozoic rocks are exposed in laterally continuous belts of outcrop around the margin of the San Juan basin. Underlying metasedimentary and plutonic rocks of the Precambrian basement are exposed in the cores of adjacent uplifts just outside the basin. The Paleozoic and Mesozoic formations in the basin were depositionally continuous with rocks of equivalent age contained in other Laramide basins of the Colorado Plateau and the Western Interior. These formations are products of sedimentary depositional systems that covered much larger areas than the Tertiary San Juan basin.

The Paleozoic rocks in the basin were deposited on a relatively stable, tectonically inactive continental platform which constituted the western margin of the continent throughout the era. Westward, across the Paleozoic continental margin, terrestrial red beds of conglomerate, sandstone, and shale intertongue with marginal marine and marine limestone, siltstone, claystone, and evaporite deposits of the Paleozoic continental slope. Sediment source areas, including the ancestral Rocky Mountains, lay within the area of the present Western Interior of the continent.

Mesozoic sediments of the Western Interior apparently were deposited differentially and sporadically through time from the Middle Triassic to the Late Cretaceous in a north-south elongate geosynclinal trough which spanned the entire Western Interior of the continent. Maximum development of the trough occurred during the Cretaceous. Sediment source areas lay west of the trough in the Cordilleran orogenic belt, which formed along the tectonically active western edge of the North American plate. Epeirogenic subsidence of the Western Interior 

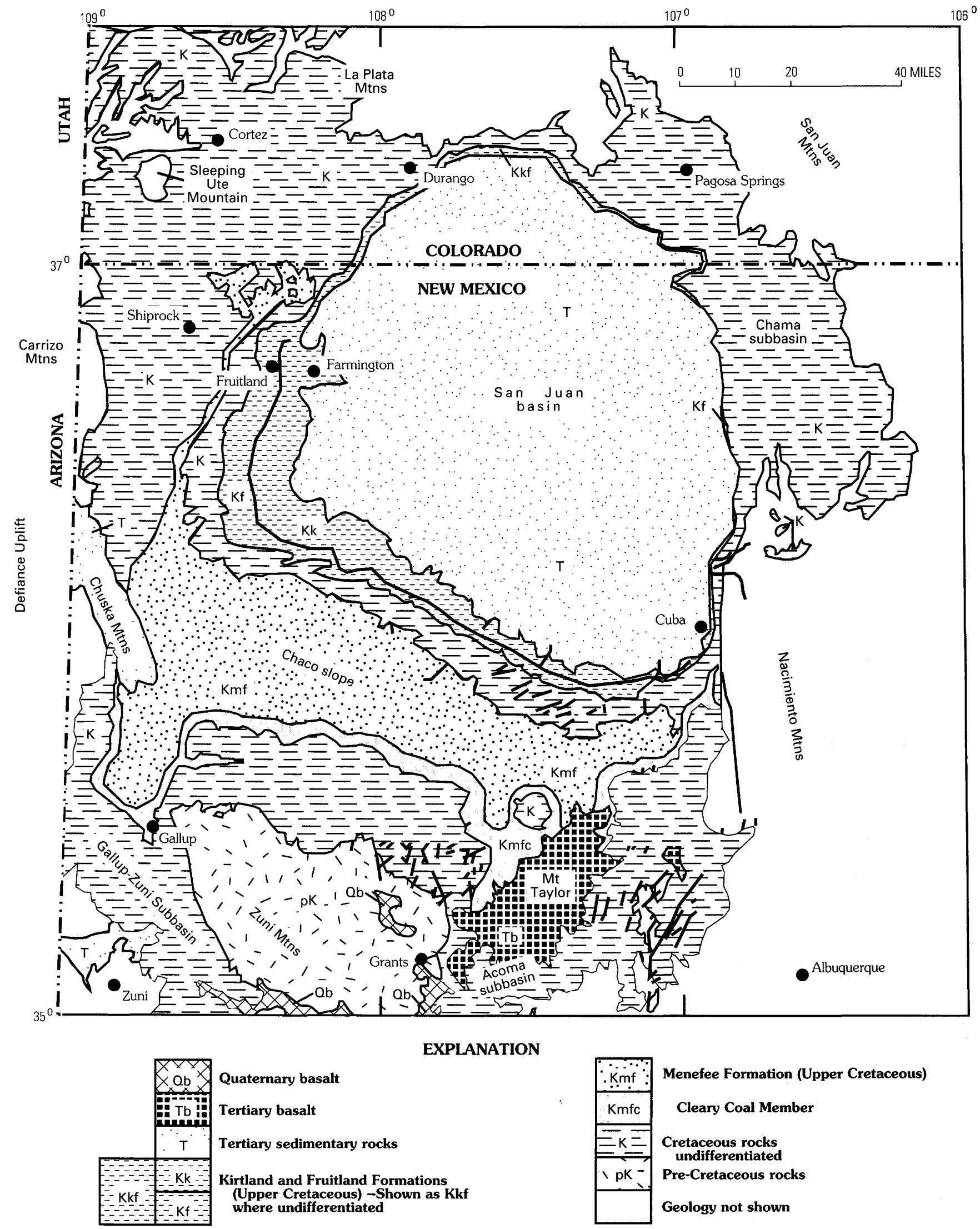

Figure A1. Generalized geologic map of the San Juan basin and adjacent region showing distribution of Cretaceous and younger rocks. (Modified from Beaumont, 1982.) 


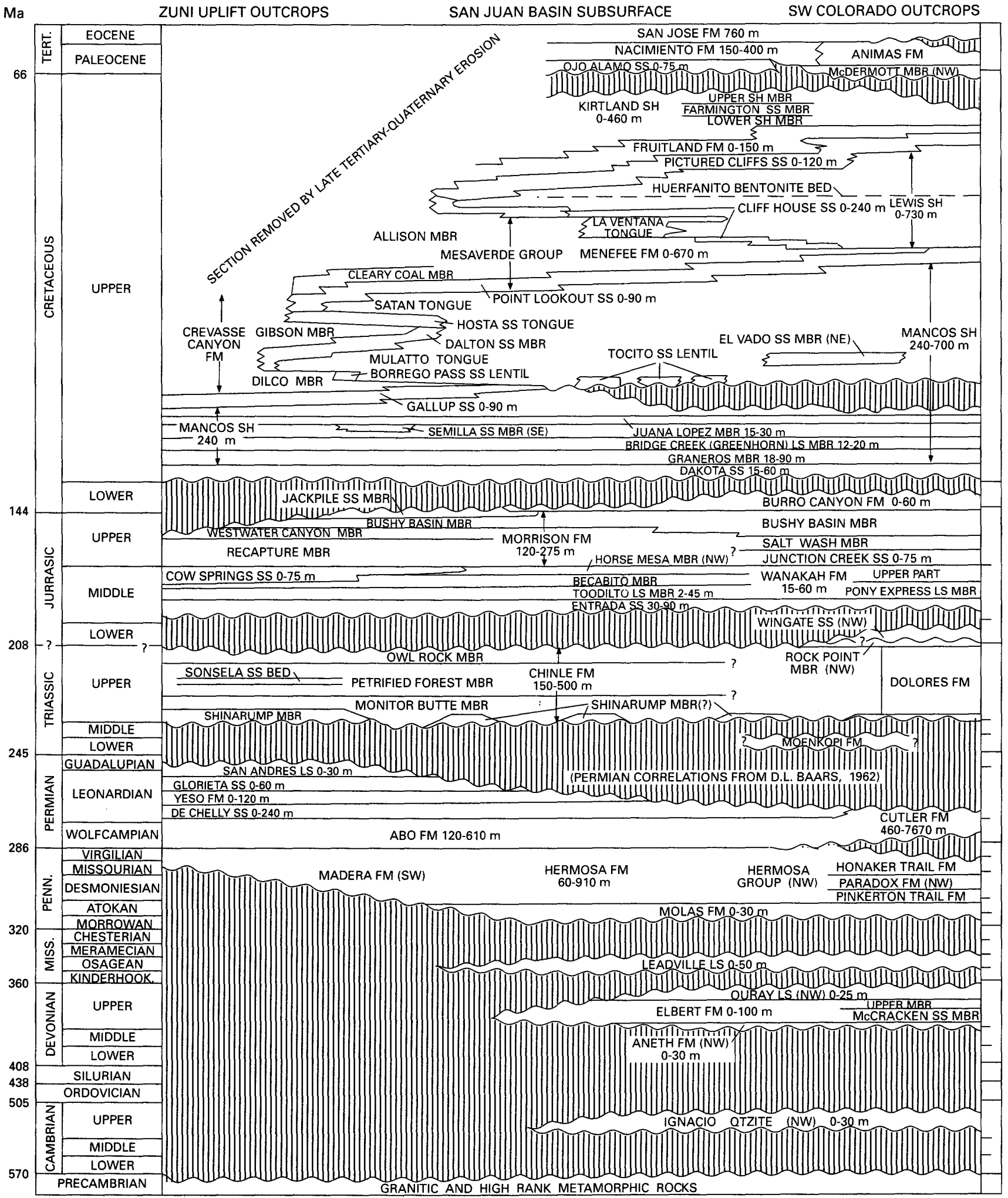

Figure A2. Stratigraphic correlation chart of the San Juan basin. (Modified from Molenaar, 1977; absolute time scale from Geological Society of America.) 


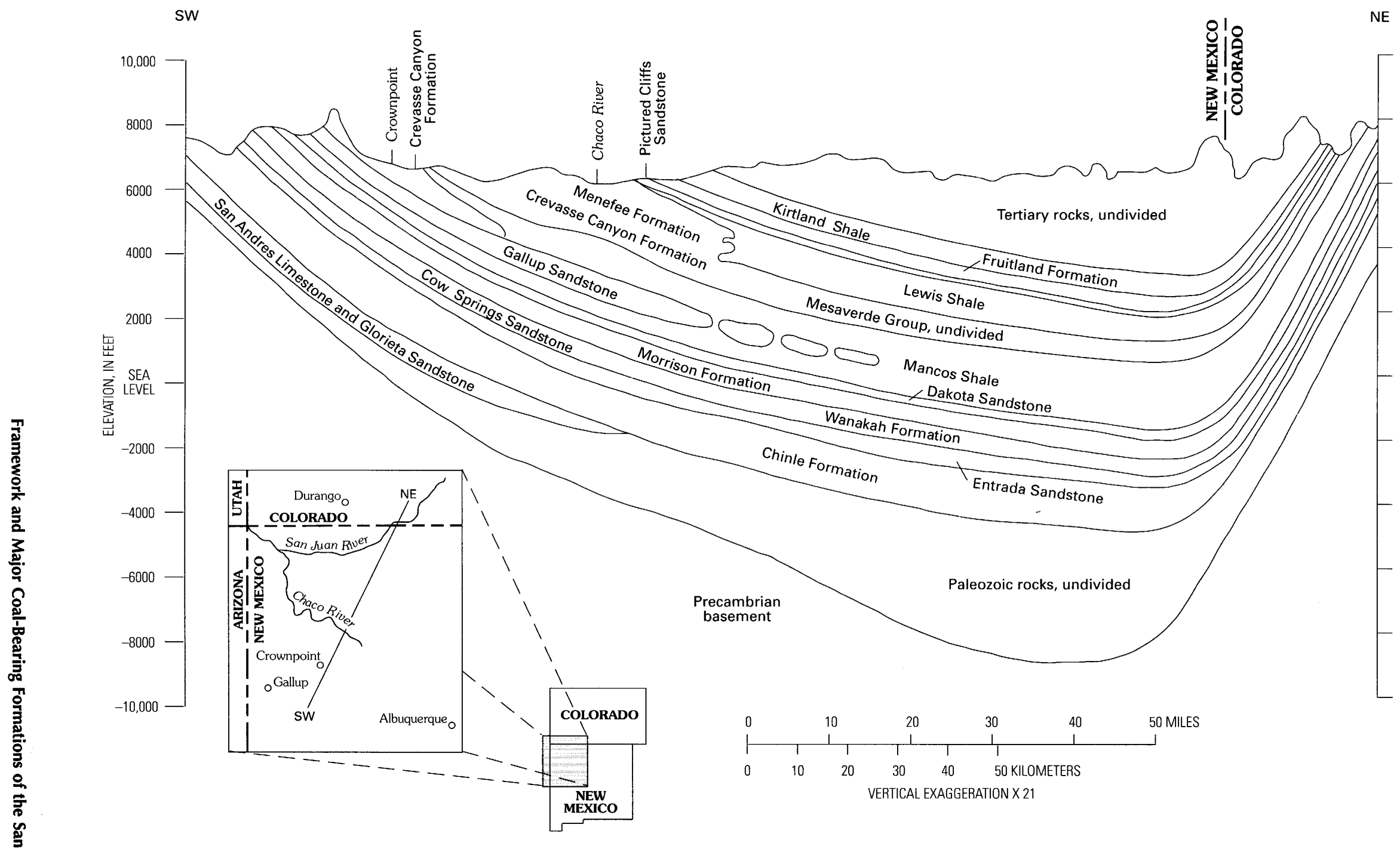

Figure A3. Diagrammatic cross section of the San Juan basin. (Modified from Stone and others, 1983, fig. 10.) 


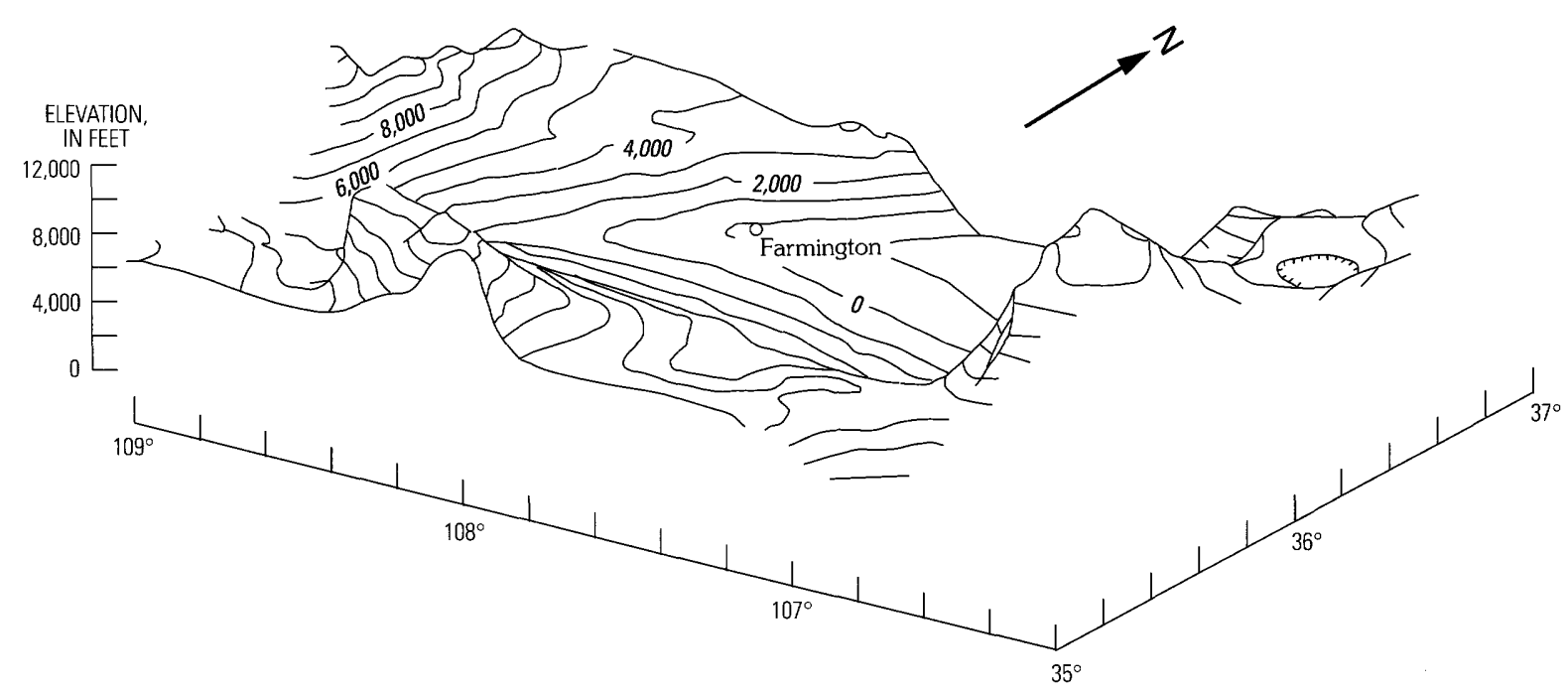

Figure A4. Structure contour perspective on the base of the Dakota Sandstone, San Juan basin. (Modified from Thaden and Zech, 1984.)

apparently resulted from tectonic compression of the crust between the Cordilleran region and the interior craton east of the trough as the western edge of the plate collided with the developing Cordilleran magmatic arc of the orogenic belt.

Western Interior trough sediments of Triassic age derived from Cordilleran source areas consist dominantly of conglomerate, sand, silt, and clay deposited in fluvial, lacustrine, and eolian depositional environments. These sediments were deposited in a number of large subbasins, which formed in the Western Interior as a result of differential subsidence of individual crustal blocks within the geosynclinal trough. On the northern and southern ends of the trough near the continental margins, Triassic and Jurassic continental sediments locally intertongue with marine sequences deposited during periodic marine invasions into the trough.

During the Cretaceous Period, maximum subsidence of the trough and high sea levels, resulting from an increase in the rate of sea-floor spreading east of the continent, caused marine waters to cyclically invade the entire Western Interior from its northern and southern ends to form the Rocky Mountain seaway. During this period the rate of sedimentary infill of the trough was at its maximum. Sediments consisting of cobbles, sand, carbonaceous silt, clay, and peat were deposited on deltas, coastal plains, and offshore areas along the western margin of the seaway. Generally, coarse-grained terrestrial lithofacies in the west change into or intertongue with fine-grained, timeequivalent, marine lithofacies to the east across the trough. During this cycle of sedimentation, the Cretaceous shoreline underwent at least five major transgressions and regressions (Molenaar, 1983).

Climatic conditions during the period of Cretaceous deposition were conducive to the luxuriant growth of vegetation along margins of the seaway, resulting in local thick accumulations of peat and organic debris. These organic accumulations later compacted and altered to form the coal deposits preserved today in the San Juan basin and other Laramide basins of the Western Interior.

\section{COAL GEOLOGY}

The geographic locations and stratigraphic positions of coal beds of Late Cretaceous age in the San Juan basin were determined by the changing location of the shoreline along the western border of an epicontinental sea of that time. The coal deposits originated from thick accumulations of detrital organic matter (peat) in paludal environments on deltas and lower coastal plains. These environments were present in the San Juan basin as a result of periodic transgression and regression of the Cretaceous sea. The coal beds tend generally to be elongate toward the northwest, parallel to the shoreline.

With each successive major transgressive-regressive cycle, the northwest-trending shoreline retreated progressively farther northeast until, by the end of the Cretaceous Period, the sea had withdrawn from the region completely (fig. A5). At times the shoreline remained in one position long enough for thick coastal sandstone to be deposited and for adjacent swamps to maintain the delicate balance necessary for thick deposits of organic matter to accumulate and alter to coal. The sedimentary record of each major transgressive-regressive cycle includes at least one coal-bearing unit. Although nearly all Cretaceous rocks in the San Juan basin contain some coal, the major coalbearing units of Cretaceous age in the basin are, in ascending order, the Crevasse Canyon, Menefee, and Fruitland Formations. 


\section{EXPLANATION}

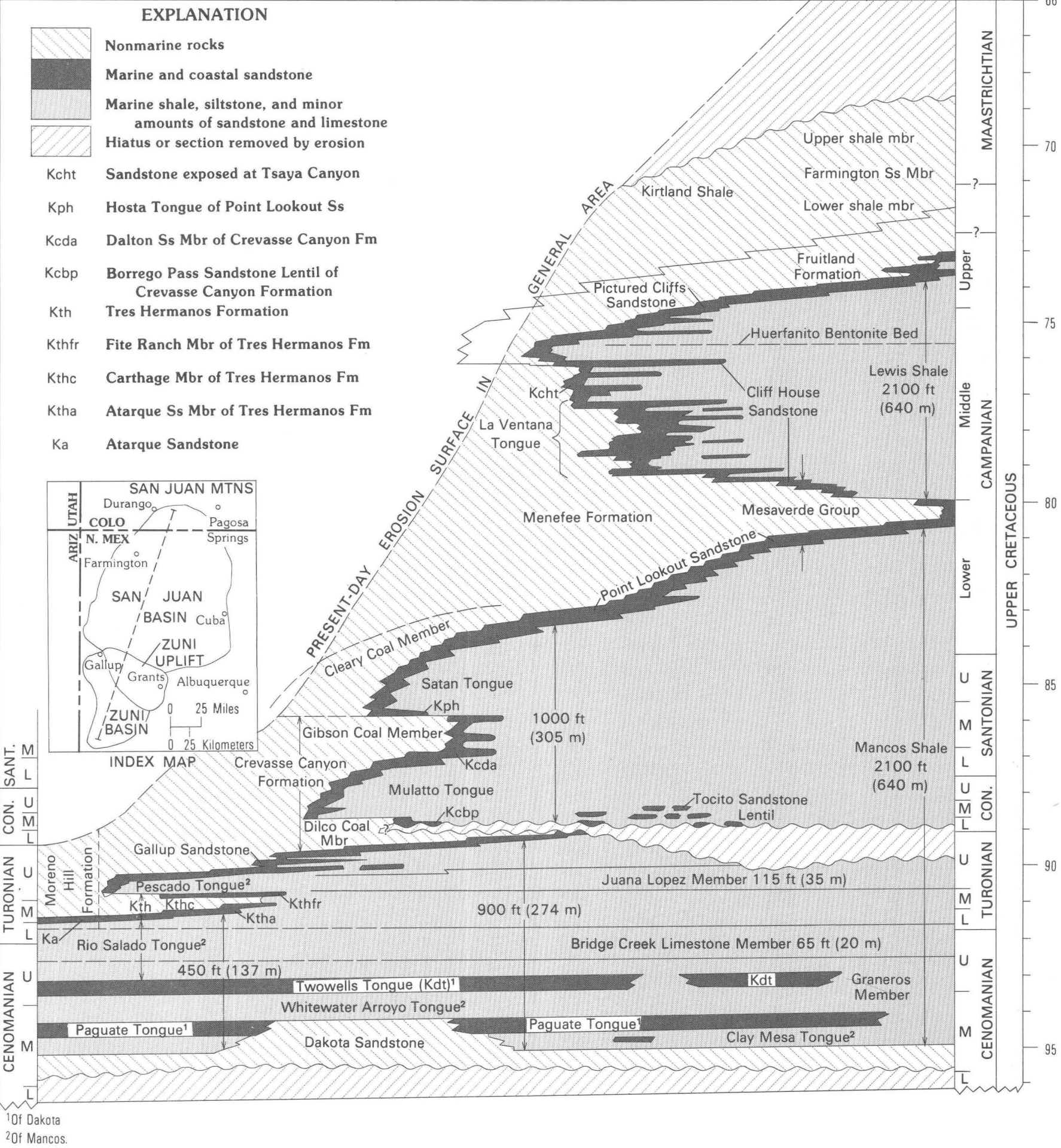

Figure A5. Time-stratigraphic geologic section of Upper Cretaceous rocks, San Juan basin (Molenaar and Baird, 1991). 

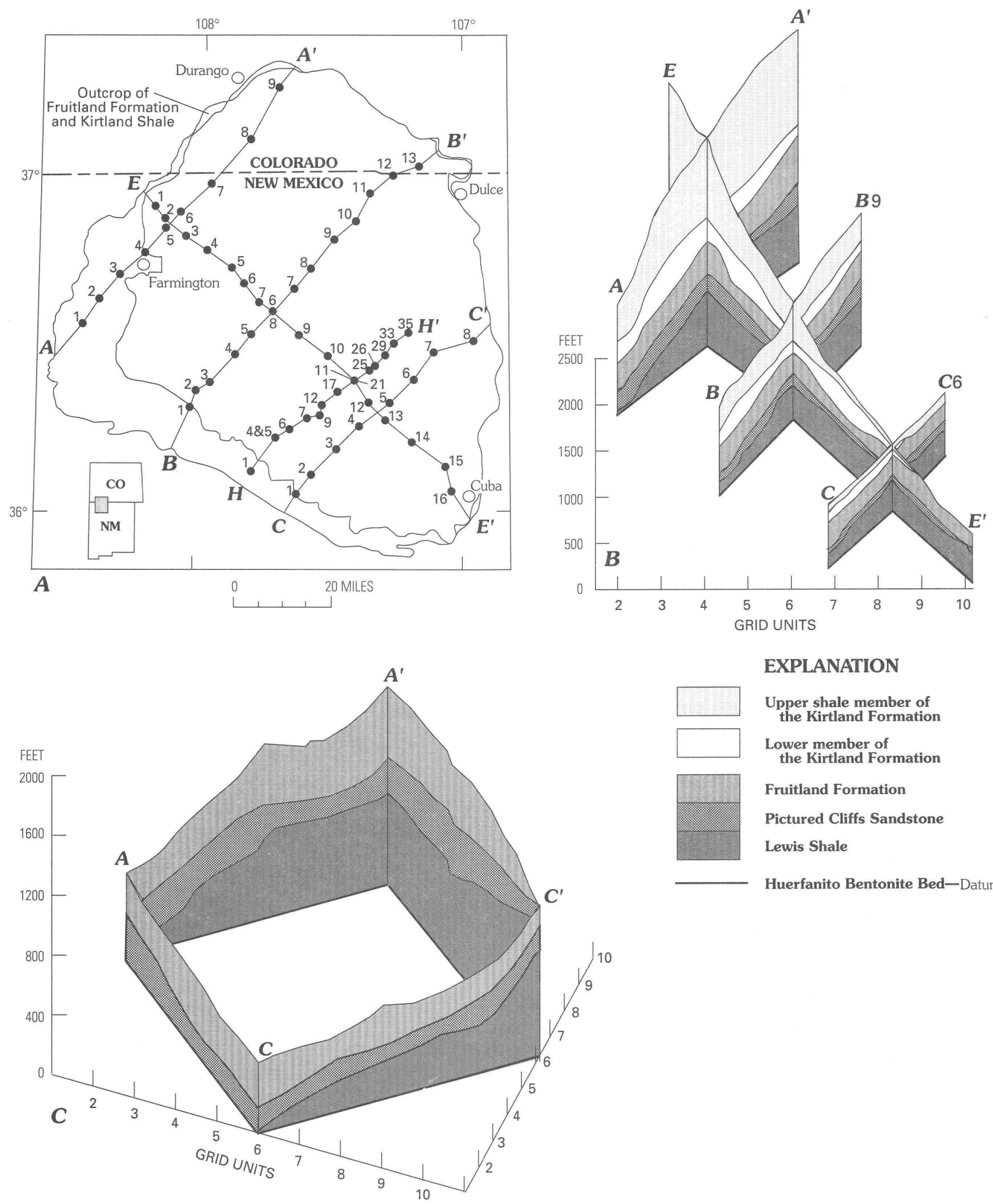

EXPLANATION

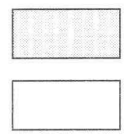

Upper shale member of the Kirtland Formation

Lower member of the Kirtland Formation

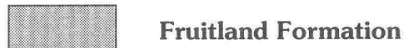

Pictured Cliffs Sandstone

Lewis Shale

Huerfanito Bentonite Bed-Datum

Figure A6. Cross sections $A-A^{\prime}$ through $E-E^{\prime}$, showing stratigraphic rise of the Pictured Cliffs Sandstone across the San Juan basin (from Fassett and Hinds, 1971, pl. 2); also shown are locations of section $H-H^{\prime}$ and of drill holes $H-1$ through $H-35$, presented in figure A7. A, Index map showing lines of sections. Numbered points indicate locations of oil and gas wells; electric logs of these wells were used in constructing stratigraphic cross sections. $B$ and $C$, Fence diagrams based on sections $A-A^{\prime}$ through $E-E^{\prime}$. Vertical scales show feet above datum (the Huerfanito Bentonite Bed); horizontal scale shows grid unit across basin (approximately $10 \mathrm{mi}$ each). 
Coal beds in the Dilco and Gibson Coal Members of the Crevasse Canyon Formation (fig. A5) have long been mined in the southwest part of the San Juan basin. Where the Gibson Member of the Crevasse Canyon Formation and the Cleary Coal Member of the Menefee Formation merge, several coal beds are currently being strip-mined at the McKinley Mine (fig. A8, No. 6), northwest of Gallup (Wilson, 1977, p. 255).

The Menefee Formation, in general, contains coal beds at its base and its top but is essentially barren in the middle part. In the northwestern part of the basin, the coal-bearing units are called the lower and upper coalbearing members (Hayes and Zapp, 1955). In the southern part of the basin, the Menefee is divided into two members, a lower coal-bearing member, called the Cleary Coal Member, and the overlying Allison Member, which generally includes some coal at the top. Miller (1984) has named the coal-bearing interval at the top of the formation the La Vida Beds of the Allison Member. The upper coal-bearing unit generally underlies, or intertongues with, the lower part of the overlying Cliff House Sandstone. Along the east side of the San Juan basin the lower coal member crops out and can be traced from the southeast northward, nearly to the State line. Along outcrops on the west side of the basin the Menefee Formation in most places includes only a few coal beds except locally where it intertongues with overlying or underlying sandstones (Beaumont, 1971, p. 23). North of the San Juan River the number and thickness of coal beds increase in both coalbearing members.

A distinctive marker bed, called the Huerfanito Bentonite Bed (fig. A5) of the Lewis Shale, fell as volcanic ash into the Late Cretaceous marine seaway between the time of deposition of Cliff House transgressive sands and the final regression marked by the Pictured Cliffs sands. The Huerfanito Bentonite Bed is a time-stratigraphic marker named and traced in the subsurface throughout the San Juan basin by Fassett and Hinds (1971). They used this stratigraphic marker to demonstrate the stratigraphic rise of the Pictured Cliffs Sandstone as the sea retreated northeastward, and the influence of this rise on coal beds in the overlying Fruitland Formation. Figure A6 shows the lines of section constructed by Fassett and Hinds (1971, plate 2) using the Huerfanito Bentonite Bed as a datum to show the stratigraphic rise northeastward of the Pictured Cliffs and the change in thickness of the overlying Kirtland Shale. Figures A6B and $C$ are computer-generated fence diagrams, based on cross sections $A-A^{\prime}$ through $E-E^{\prime}$, to illustrate the concept of the stratigraphic rise. Panel $E-E^{\prime}$ in figure $A 6 B$ and $A-C$ in figure $A 6 C$ parallel the Cretaceous shoreline and suggest essentially no stratigraphic change. Figure $A 6 B$ also shows the general changes in thickness of members of the Kirtland
Shale. The Kirtland is thickest in the northern part of the basin. (See fig. $\mathrm{A} 6 B$, line $A-A^{\prime}$ ). The complicated changes in thickness of its members are discussed in detail by Fassett and Hinds (1971, p. 23-28).

The Fruitland Formation, which overlies the Pictured Cliffs Sandstone, contains by far the largest coal resources in the San Juan basin. Most of the coal occurs in lenticular beds in the lower part of the formation. Coal has been surface mined from the Fruitland near the San Juan River in the western part of the basin for many years. The thickness and distribution of coal in the Fruitland is closely related to the increase in thickness and stratigraphic rise of the Pictured Cliffs Sandstone, especially where the lower part of the Fruitland Formation intertongues with the upper part of the Pictured Cliffs. Figure A7 is a computer-generated plot of drill holes showing examples of Fruitland coal and the underlying Pictured Cliffs in a line extending northeastward across the basin for about 40 miles. In the plot, the datum is the base of the Pictured Cliffs Sandstone, and the data are interpreted from geophysical logs of oil- and gas-test holes. Typically, the coal beds are highly lenticular and difficult to correlate between drill holes. The thickest coal beds are generally near the base of the Fruitland, but they can be correlated with certainty only where drill holes are closely spaced. For these reasons, coal resource calculations can only be made for coal zones rather than for individual beds. The coal beds as shown in figure A7 are, in places, at least 20 feet thick, and there is a suggestion of two coal zones between drill holes H-9 to H-35. The thickest Fruitland coal in the basin is south of Durango, Colo., near Carbon Junction, where one bed consists of 80 feet of interbedded coal and partings (Zapp, 1949).

The San Juan basin is subdivided into 21 individual coalfields or coal areas underlain by strippable low-sulfur coal (fig. A8). Those fields or areas in which the Fruitland Formation is the current producer are the Navajo field and the Fruitland, Bisti, and Star Lake areas. The remaining fields or areas have coal resources-either developed or undeveloped-in the Mesaverde Group and its equivalents, the most notable being the Gallup field.

\section{REFERENCES CITED}

Baars, D.L., 1962, Permian System of Colorado Plateau: American Association of Petroleum Geologists Bulletin, v. 46, p. 149-218.

Beaumont, E.C., 1971, Stratigraphic distribution of coal in the San Juan basin, in Shomaker, J.W., Beaumont, E.C., and Kottlowski, F.E., eds., Strippable low-sulfur coal resources of the San Juan basin in New Mexico and Colorado: New Mexico Bureau of Mines and Mineral Resources Memoir 25 , p. $15-30$. 


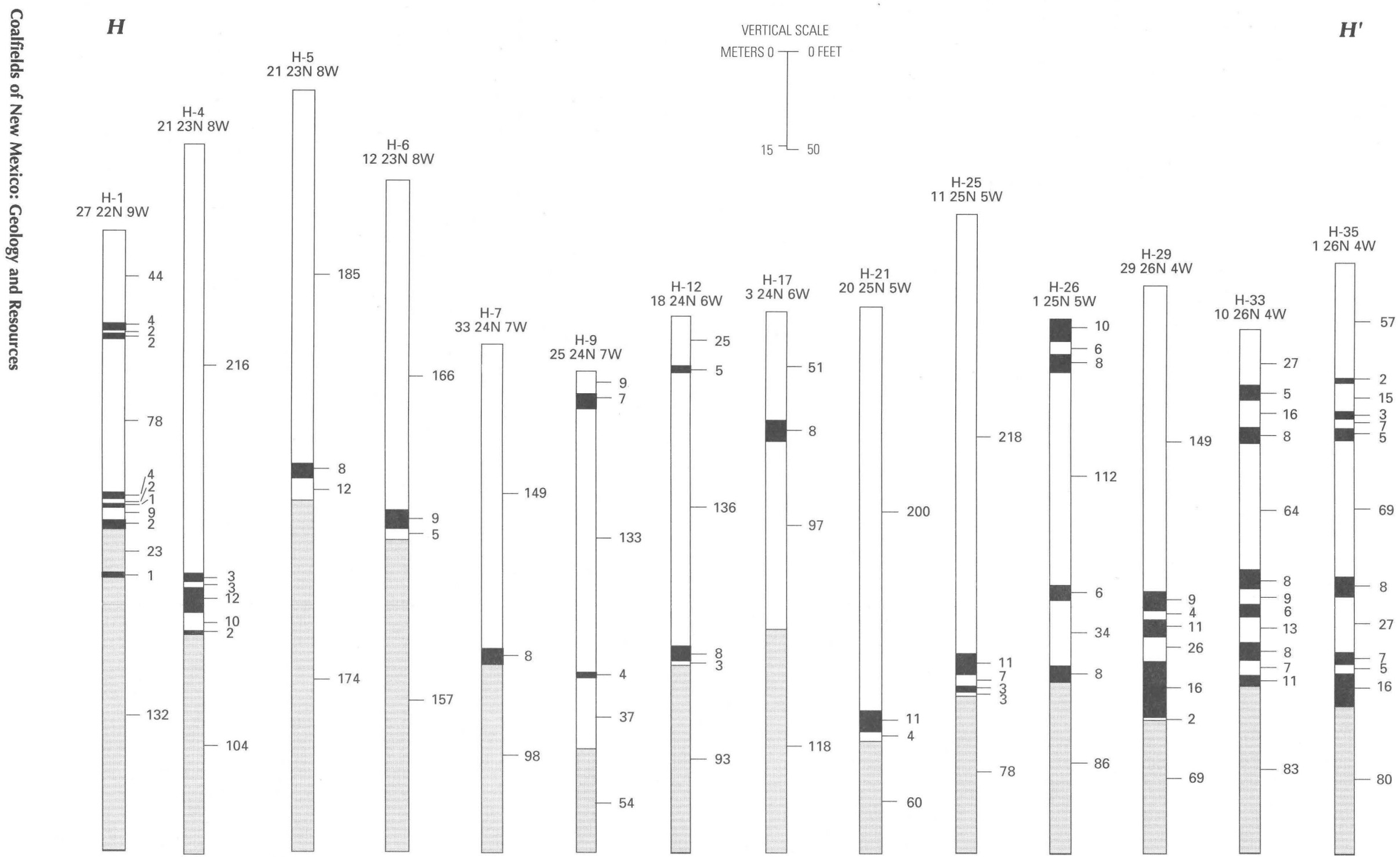

Figure A7. Correlation of well logs along section $\mathrm{H}-\mathrm{H}^{\prime}$ (fig A6A) showing lenticular nature of the coal beds (black) within the Fruitland Formation (no pattern). Stippled pattern represents Pictured Cliffs Sandstone; datum is the base of the Pictured Cliffs. Numbers next to columns give bed thicknesses in feet. 


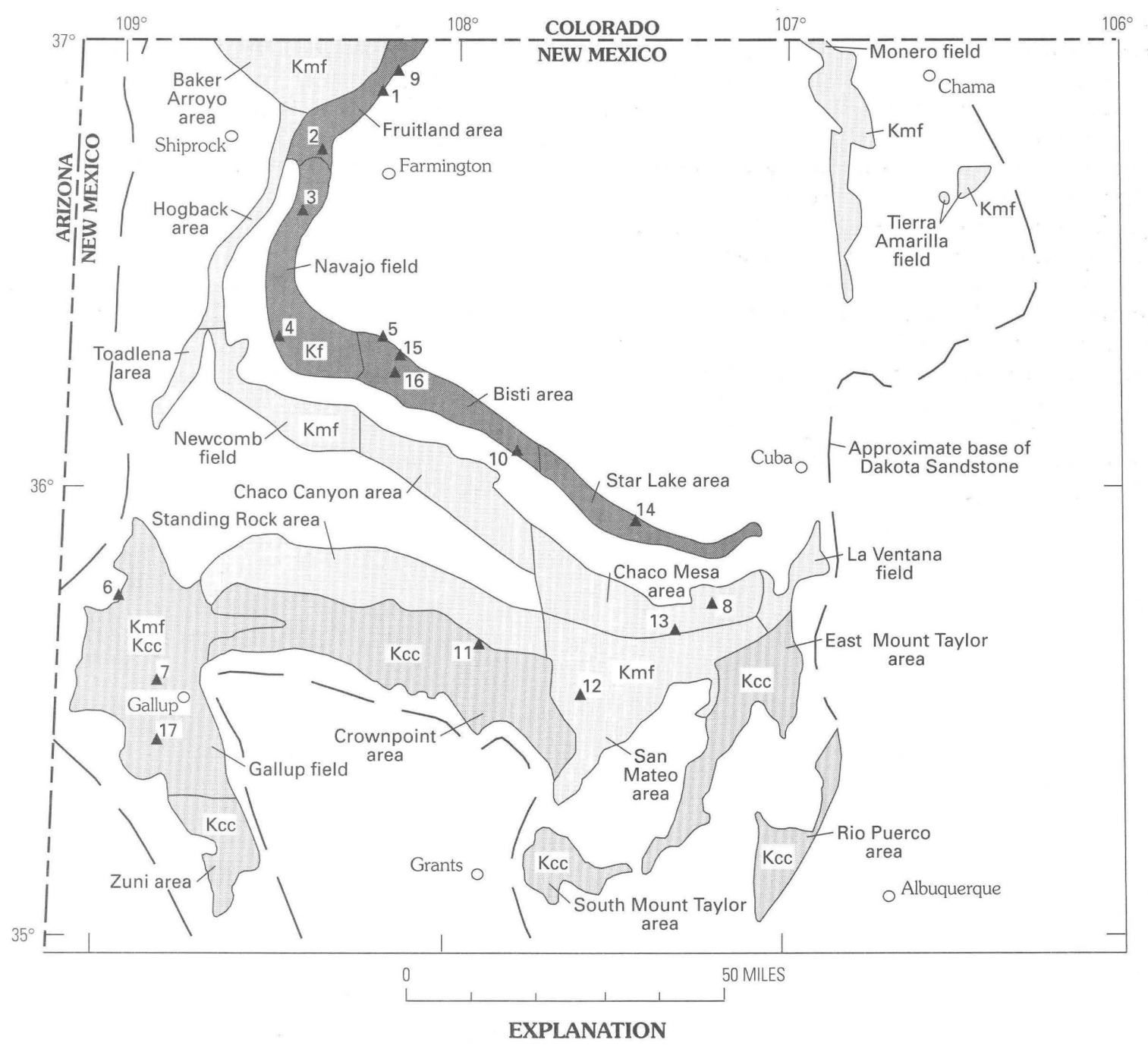

\begin{tabular}{|c|c|c|c|c|}
\hline & & \multicolumn{3}{|c|}{ Active Mines, Permitted Mines, and Mine Permits in Progress } \\
\hline $\mathrm{Kf}$ & Fruitland Formation & 1. Black Diamond & 7. Mentmore & 13. La Ventana \\
\hline Kmf & Menefee Formation & 3. Navajo & 9. La Plata & 15. Gateway \\
\hline Kcc & Crevasse Canyon Formation & 4. Burnham & $\begin{array}{l}\text { 10. Gallo Wash } \\
\text { 11. South Hospah }\end{array}$ & $\begin{array}{l}\text { 16. Bisti } \\
\text { 17. Carbon No. } 2\end{array}$ \\
\hline
\end{tabular}

Figure A8. Fields, areas, and mines containing strippable low-sulfur coal in Upper Cretaceous formations of the San Juan basin. (Modified from Kottlowski and others, 1983.)

1982, Geology of New Mexico coal deposits and geologic setting for field trip, in Coal-bearing sequences and modern concepts for exploration and development: American Association of Petroleum Geologists short course, March 1982.

Fassett, J.E., and Hinds, J.S., 1971, Geology and fuel resources of the Fruitland Formation and Kirtland Shale of the San Juan basin, New Mexico and Colorado: U.S. Geological Survey Professional Paper 676, 76 p.

Finch, W.I., Huffman, A.C., Fassett, J.E., eds., 1989, Coal, uranium, and oil and gas in Mesozoic rocks of the San Juan basin-Anatomy of a giant energy-rich basin, in Coal and hydrocarbon resources of North America: International
Geological Congress, 28th, Field Trip Guidebook T120, v. $1,99 \mathrm{p}$.

Hayes, P.T., and Zapp, A.D., 1955, Geology and fuel resources of the Upper Cretaceous rocks of the Barker dome-Fruitland area, San Juan County, New Mexico: U.S. Geological Survey Oil and Gas Investigations Map OM-144, scale $1: 62,500$.

Kottlowski, F.E., Campbell, F.W., Roybal, G.H., and Hatton, K.S., 1983, New Mexico, in 1983 Keystone coal industry manual: New York, McGraw-Hill, p. 577-586.

Miller, R.L., 1984, Subdivisions of the Menefee Formation and Cliff House Sandstone (Upper Cretaceous) in southwest San Juan basin, New Mexico: U.S. Geological Survey Bulletin 1537-A, A29-A53. 
Molenaar, C.M., 1977, San Juan basin time-stratigraphic nomenclature chart, in Fassett, J.E. and James, H.L., eds., Guidebook of San Juan basin III, northwestern New Mexico: New Mexico Geological Society, 28th Field Conference, 1977, p. xii.

1983, Major depositional cycles and regional correlations of Upper Cretaceous rocks, southern Colorado Plateau and adjacent areas, in Reynolds, M.W., and Dolly, E.D., eds., Mesozoic paleogeography of the west-central United States: Denver, Colo., Rocky Mountain Section, Society of Economic Paleontologists and Mineralogists, p. 201-224.

Molenaar, C.M., and Baird, J.K., 1991, Stratigraphic cross sections of Upper Cretaceous rocks in the northern San Juan basin, Southern Ute Indian Reservation, chapter $\mathrm{C}$ of Geology and mineral resources of the Southern Ute Indian Reservation: U.S. Geological Survey Professional Paper 1505-C, p. C1-C12.
Stone, W.J., Lyford, F.P., Frenzel, P.F., Mizell, N.H., and Padgett, E.T., 1983, Hydrogeology and water resources of the San Juan basin, New Mexico: New Mexico Bureau of Mines and Mineral Resources Hydrologic Report 6, 70 p.

Thaden, R.E., and Zech, R.S., 1984, Preliminary structure contour map on the base of the Cretaceous Dakota Sandstone in the San Juan basin and vicinity, New Mexico, Arizona, Colorado, and Utah: U.S. Geological Survey Miscellaneous Field Studies Map MF-1673, scale 1:500,000.

Wilson, J.C., 1977, The McKinley Mine, in Fassett, J.E., and James, H.L., eds., Guidebook of San Juan basin III, northwestern New Mexico: New Mexico Geological Society, 28th Field Conference, 1977, p. 253-255.

Zapp, A.D., 1949, Geology and coal resources of the Durango area, La Plata and Montezuma Counties, Colorado: U.S. Geological Survey Oil and Gas Investigations Preliminary Map OM-109, scale 1:31,680. 
Chapter B

\section{Coal Resources of the San Juan Basin}

By LAURA R. H. BIEWICK, ANTOINETTE L. MEDLIN, JUDY F. HUNTER, and KATHLEEN K. KROHN,

A demonstration of the capabilities of the

National Coal Resources Data System

U.S. GEOLOGICAL SURVEY BULLETIN 1972

COALFIELDS OF NEW MEXICO: GEOLOGY AND RESOURCES 


\title{
CONTENTS
}

\author{
Abstract 17 \\ Introduction 17 \\ Procedures 17 \\ Data preparation 17 \\ Boundary definition 19 \\ Resource calculations 25 \\ Results 25 \\ Considerations and issues 27 \\ References cited $\mathbf{3 3}$
}

FIGURES

B1-B8. Maps of the San Juan basin showing:

B1. Areas defined for estimates of coal resources in the Fruitland and Menefee Formations 18

B2. Isopachs of total coal in the Fruitland Formation 19

B3. Thickness of overburden on top of coal in the Fruitland Formation 20

B4. Isopachs of total coal in the Menefee Formation 21

B5. Thickness of overburden on top of coal in the Menefee Formation 22

B6. Digitized boundaries showing generalized land categories in areas underlain by the Fruitland Formation 23

B7. Digitized boundaries showing generalized land categories in areas underlain by the Menefee Formation 24

B8. Number of coal horizons in the Fruitland Formation 27

\section{TABLES}

B1. Estimates of Federally owned subbituminous coal resources in the Fruitland Formation, San Juan basin, New Mexico 28

B2. Estimates of Federally owned subbituminous coal resources in the Menefee Formation, San Juan basin, New Mexico 


\title{
Coal Resources of the San Juan Basin
}

\author{
By Laura R.H. Biewick, Antoinette L. Medlin, Judy F. Hunter, and Kathleen K. Krohn
}

\begin{abstract}
A working data base of stratigraphic information for the Upper Cretaceous Menefee and Fruitland Formations, the two major coal-bearing units within the San Juan basin, was retrieved from the NCRDS (National Coal Resources Data System) master data base for New Mexico using the PACER data storage and retrieval system on a 32-bit minicomputer. These data were reviewed, and data from other sources were coded and added. Then subsets of data were retrieved, representing drill holes that had penetrated the entire thickness of either of the formations within the digitized boundary areas.

The coal resources of both formations were calculated from this data set using GARNET, one of the graphic analysis and volume calculation programs of NCRDS. Because of the lenticular nature of the coal beds and the large area under study, the resource assessments were made for total coal within each of the formations, rather than for individual coal beds.

The GARNET program uses a point location file, a grid file of coal thickness, and a boundary file to calculate coal volume; then it multiplies the volume by a density factor, which depends on the coal's rank, to produce a coal tonnage estimate. These estimates, for the New Mexico part of the San Juan basin, are 168 billion short tons of coal in the Fruitland Formation and 21 billion short tons of coal in the Menefee Formation. Each estimate is presented in three ways: (1) as a single resource tonnage; (2) as tonnages in the measured, indicated, inferred, and hypothetical categories; (3) as tonnages in various generalized land ownership categories; and (4) as tonnages in various overburden categories by township, range, and county. The GARNET program was also used to produce coal isopach, overburden, and generalized land ownership maps.
\end{abstract}

\section{INTRODUCTION}

The two predominant coal-bearing sequences in the San Juan basin, the Upper Cretaceous Menefee and Fruitland Formations (fig. B1), have been studied using traditional and computerized methods. This study had four objectives:

Manuscript approved for publication January 10, 1991.
1. To estimate the total coal resources in the Menefee and Fruitland Formations in the San Juan basin, within the limitations of the available data.

2. To estimate the approximate amounts of the total coal resources that occur in areas within various generalized categories of coal mineral rights ownership.

3. To estimate the amounts of the total coal resources within various categories of overburden thickness.

4. To demonstrate the capabilities of the U.S. Geological Survey's National Coal Resources Data System (NCRDS) for coal resource calculations.

The coal resource estimates presented in this chapter were calculated using the data and programs of the NCRDS. PACER is the data base formatting, storage, and retrieval program and GARNET is the graphic analysis and volume calculation program of NCRDS. A 32-bit minicomputer was used for this study. All of the techniques and procedures illustrated here can be applied at various scales, to areas ranging from federal lease tracts to whole States or regions. Pertinent factors are the amount, reliability, and distribution of available data; the accuracy of boundary definitions; and the categories of resource calculations required.

\section{PROCEDURES}

\section{Data Preparation}

A working file of stratigraphic information for the San Juan basin was retrieved from the NCRDS master data base for New Mexico (NMSTRAT) using PACER. These data had been compiled from well logs by geologists of the U.S. Geological Survey (USGS) and the New Mexico Bureau of Mines and Mineral Resources. Additional data from recent studies and other sources were coded and added to the working file by the USGS project geologists and NCRDS staff. The geologists checked the designations of stratigraphic units and, where necessary, corrected the working file using the PACER updating capability. Data from this working file were used to produce several products for the geologists' analyses and approval. 


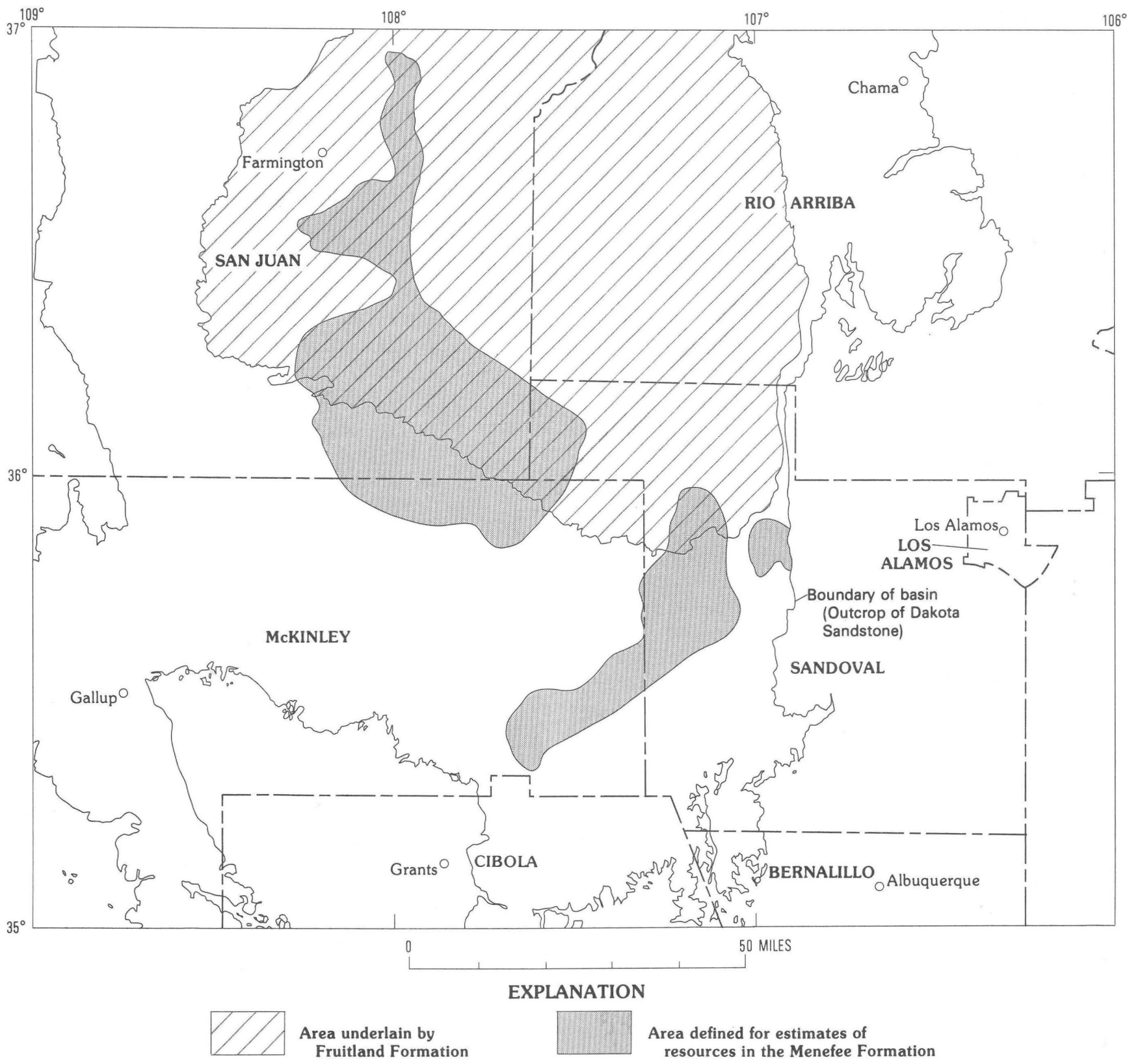

Figure B1. Location of areas defined for estimates of coal resources in the Fruitland and Menefee Formations in the San Juan basin, New Mexico. Area of the Fruitland is bounded by the formation's outcrop; area shown for the Menefee is bounded by a limit-of-estimates line based on available drill hole data.

Because the Fruitland Formation is the only formation in the San Juan basin represented by sufficient NCRDS data to allow detailed analysis, it is discussed first in this report. In order to retrieve data representative of the coal in the complete Fruitland Formation, a single PACER request was made for all drill hole records having Fruitland Formation coal lithostratigraphic units; partial sections were excluded. The request yielded 2,513 records, which were processed using standard NCRDS software to sum the thicknesses of all coal horizons from the same drill hole. This procedure resulted in total Fruitland Formation coal values for 630 randomly spaced point locations. These point locations and values were then used for input to the GARNET program for graphic display and calculation of coal tonnage estimates.

The first GARNET operation was to use the randomly spaced data point locations to create an evenly spaced grid of estimated coal thickness values. These values were then displayed as contours or isolines at intervals designated by the data analyst. An optimal grid spacing of 3 miles was selected based on the spacing of random data points over the area. The GARNET software provides two gridding algorithm options for interpolation of randomly spaced data: the quadratic algorithm for dense, evenly spaced points and 
the planar algorithm for sparse, unevenly distributed points. Fruitland Formation coal thickness data were best represented by the planar gridding option because of the distribution of the data points. The planar algorithm averages the values over a larger area than the quadratic algorithm and produced a coal isopach map satisfactory to the geologists' interpretation of the data (fig. B2).

A second PACER request to the working file retrieved values for the depth to the top (overburden) of the Fruitland Formation coal zone. These overburden values were then gridded using the GARNET program. Sufficient data points were not available to control the overburden contours at the outcrop line in three locations, so the GARNET point-editing capability was used to add control points at these three locations to generate the map shown in figure B3. This map was approved by the geologists and compares generally to figure 27 in the Fassett and Hinds (1971) study of this area.

Similar procedures were used to generate maps of the Menefee Formation coal and overburden thicknesses (figs. B4 and B5).

\section{Boundary Definition}

NCRDS includes the capability to calculate coal resource tonnage estimates from isopach maps within a boundary defining an area of interest. Very generalized land ownership categories were defined in this study to show the concept of estimating coal resources by the following areas of interest:

National parks and monuments

National forest lands

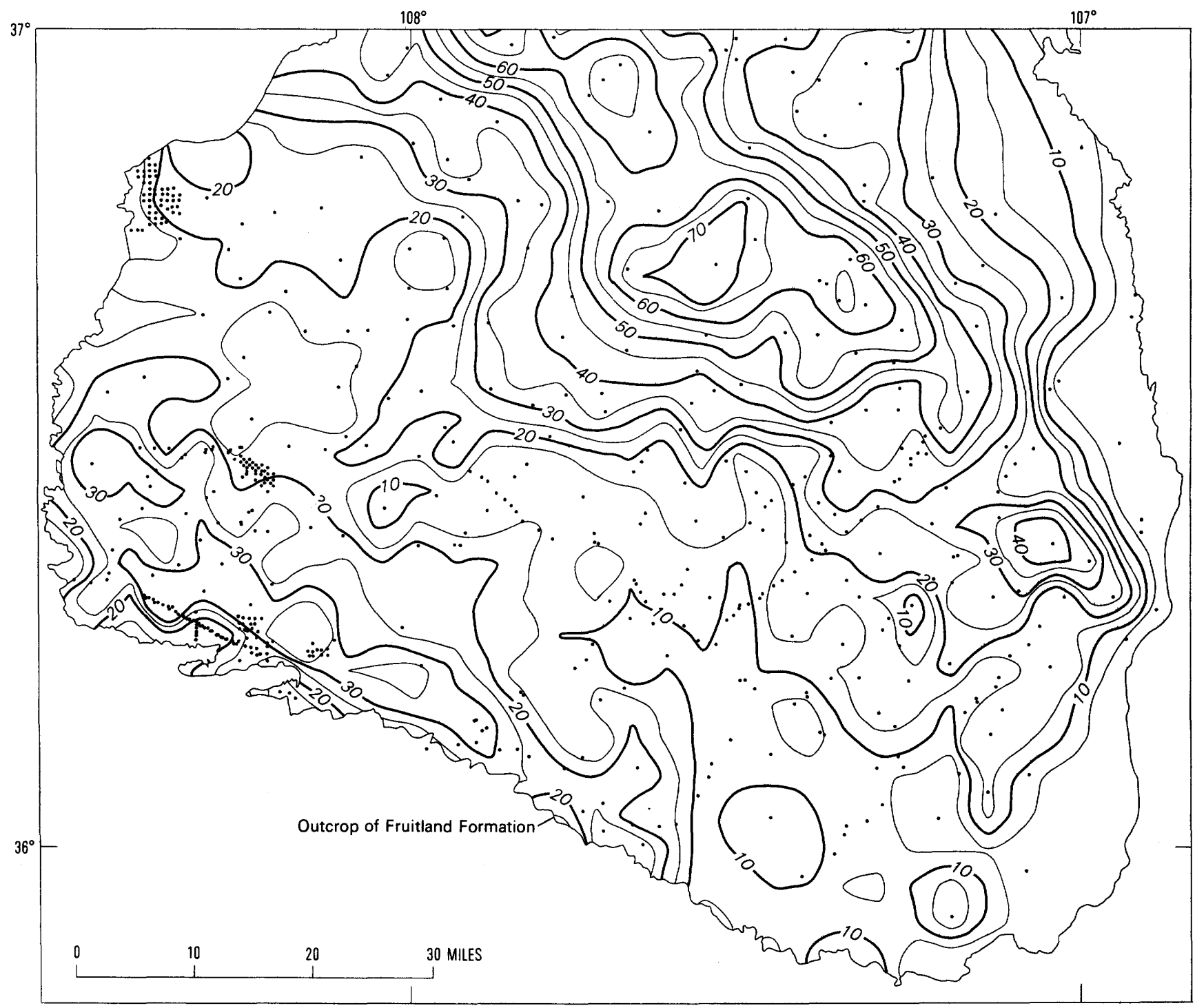

Figure B2. Isopach map of total coal in the Fruitland Formation. Dots represent data points. Contour interval 5 feet. See figure $\mathrm{B} 1$ for location of the area shown here. 


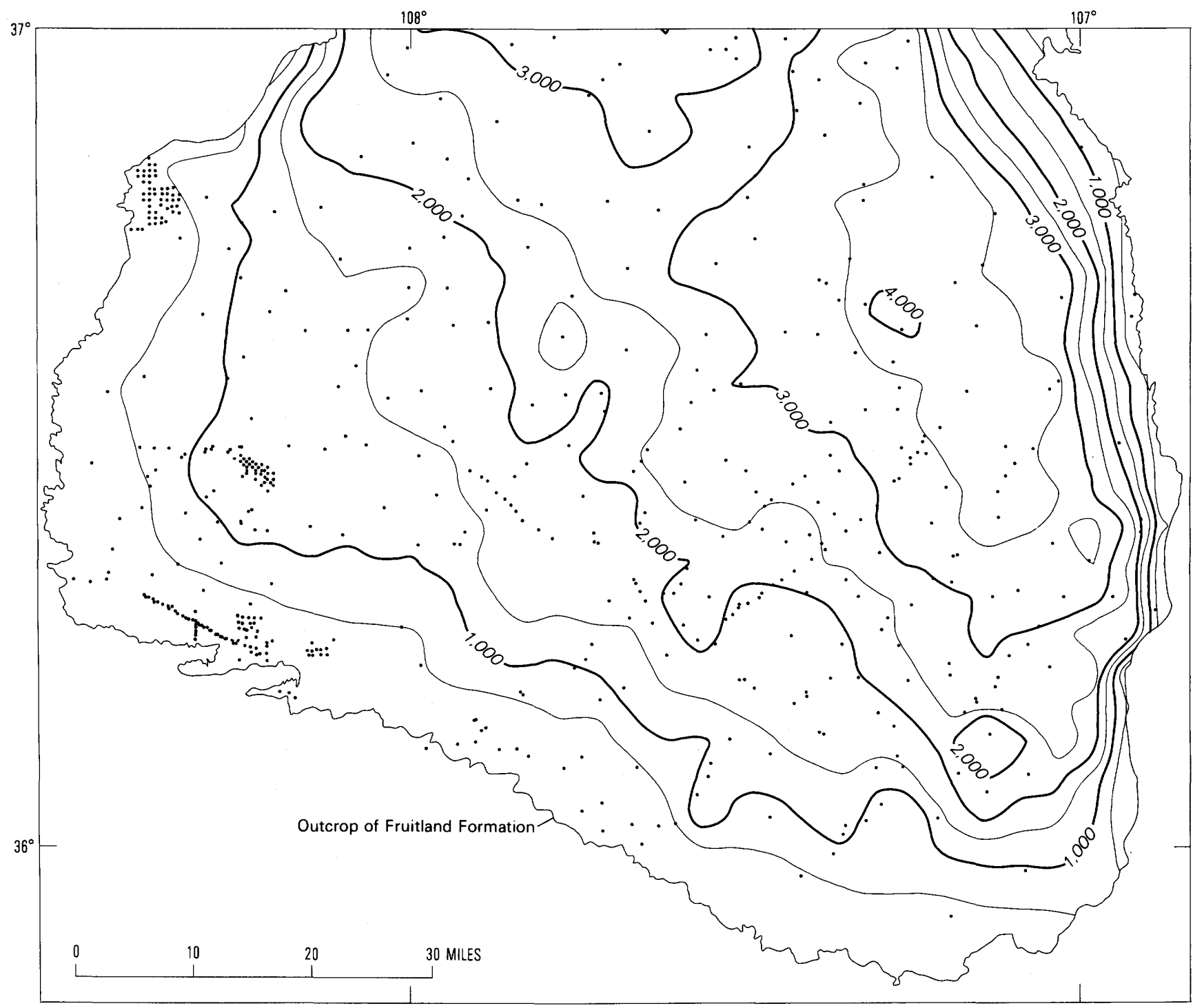

Figure B3. Thickness of overburden on top of coal in the Fruitland Formation. Dots represent data points. Contour interval 500 feet. See figure B1 for location of the area shown here. (Note: configuration of the land surface is very generally represented by the available data.)

Other federal surface and/or subsurface coal ownership Indian lands and reservations

State and privately owned lands

Counties

Digital files were created to store the boundary data that define each of these areas. Township and range boundaries determined areas used to estimate coal resource tonnages for federal lands. In all areal categories, the data were used to generate tonnage estimates in the following overburden categories:

$$
\begin{gathered}
0-500 \text { feet } \\
500-1,000 \text { feet } \\
1,000-2,000 \text { feet } \\
2,000-3,000 \text { feet }
\end{gathered}
$$

More than 3,000 feet
The GARNET program can process data and calculate results within any digital boundary that can be defined by 20,000 points or less. The boundary categories defined above provide an evaluation of approximate coal resources in the basin to illustrate the capabilities and techniques of NCRDS on a regional scale. The accuracy of the GARNET software tonnage estimates depends on the available data, the selected map scale, the digital boundary accuracy, and the chosen grid interval.

A 1:500,000 scale State of New Mexico Land Status Map (DOI, BLM edition of 1982) was used to digitize the boundaries of counties and townships and ranges, and the generalized boundaries of Indian reservations, national parks, national monuments, and national forests. Mineral ownership categories shown on figures B6 and B7 were also 


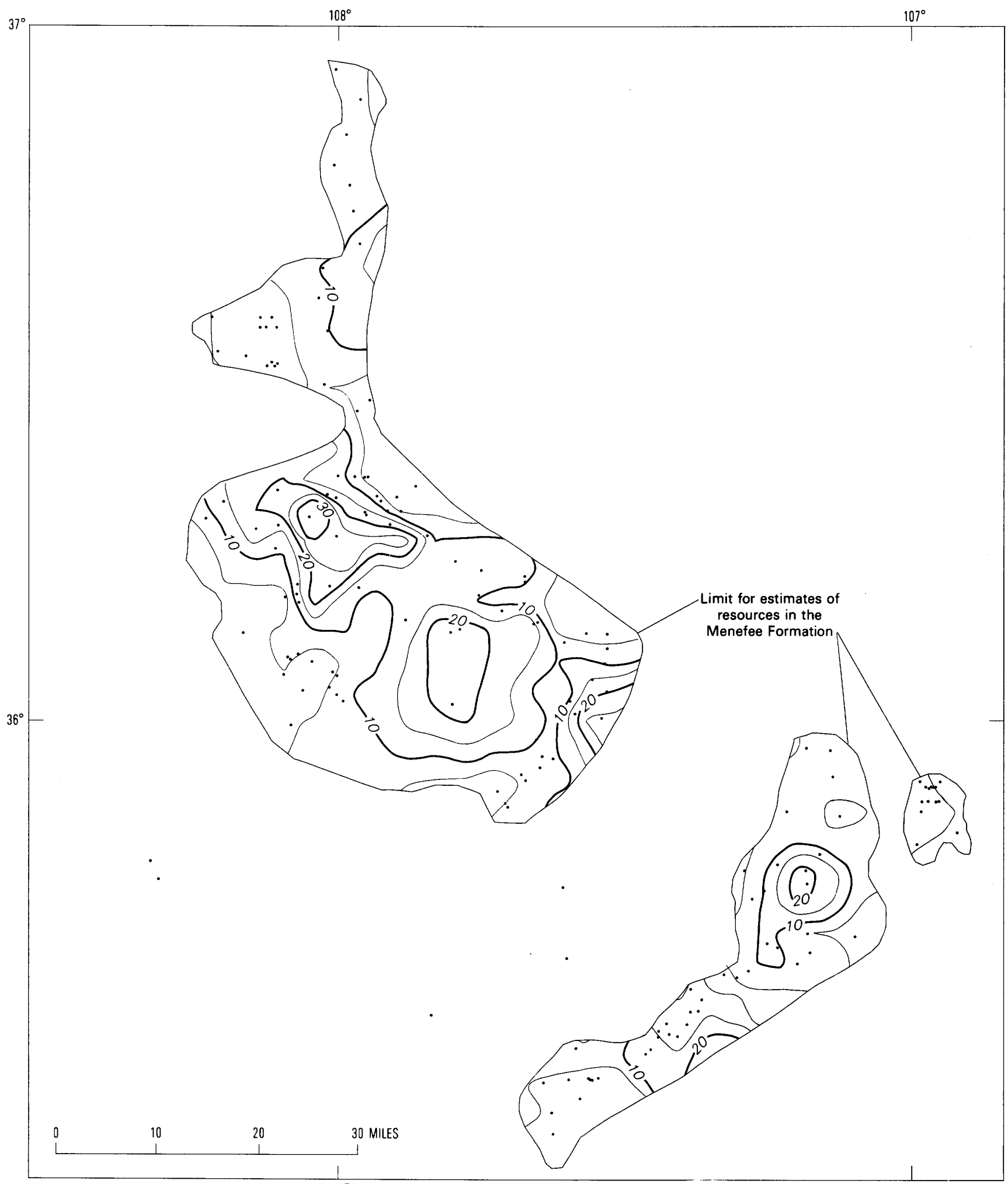

Figure B4. Isopach map of total coal in the Menefee Formation. Dots represent data points. Contour interval 5 feet. See figure B1 for location of the area shown here.

compiled from various Bureau of Land Management 1:100,000-scale land status maps (1978-1983). These boundaries are highly generalized.
Boundaries for each area of interest were digitized using equipment with an accuracy of 0.005 inch. Data from appropriate categories (political, township, range, county) 


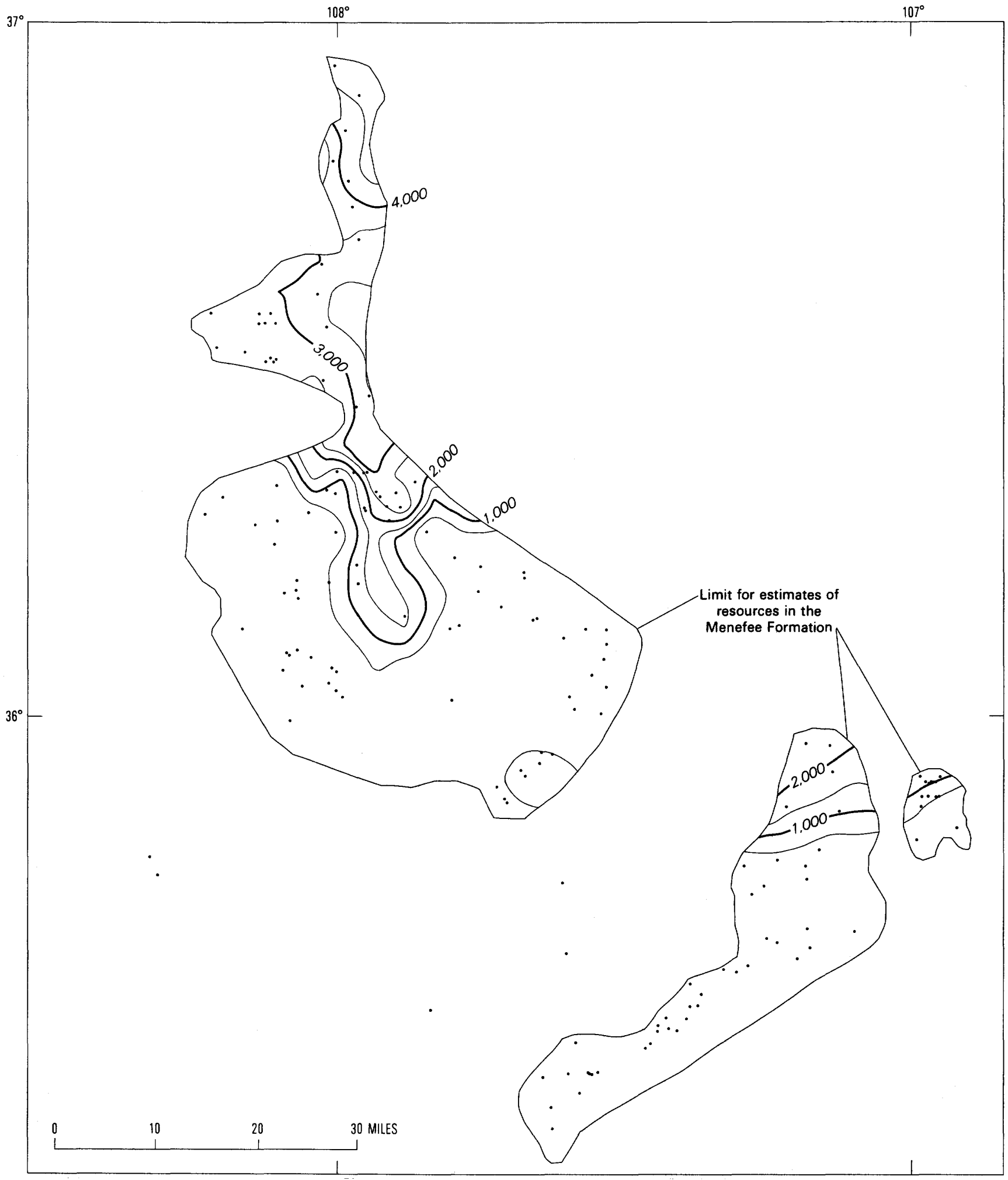

Figure B5. Thickness of overburden on top of coal in the Menefee Formation. Dots represent data points. Contour interval 500 feet. See figure B1 for location of the area shown here. (Note: configuration of the land surface is very generally represented by the available data.) 
were combined using the GARNET boundary command. The overburden category boundaries were retrieved directly by subtracting the gridded file of the elevations at the top of the coal beds from the gridded file of the surface elevations; then the overburden categories were combined with the political, land ownership, reliability, and coal thickness categories to define the final subareas for resource estimates. More than 300 subareas were defined with unique boundaries for individual coal resource tonnage calculations.

Localities of Menefee Formation coal data in NMSTRAT are densely distributed in the central and southeastern portions of the basin. In these two areas data are sufficient to allow estimates of coal resources using NCRDS. The Menefee boundary used for the isopach and overburden maps (figs. B4 and B5) represents an irregular
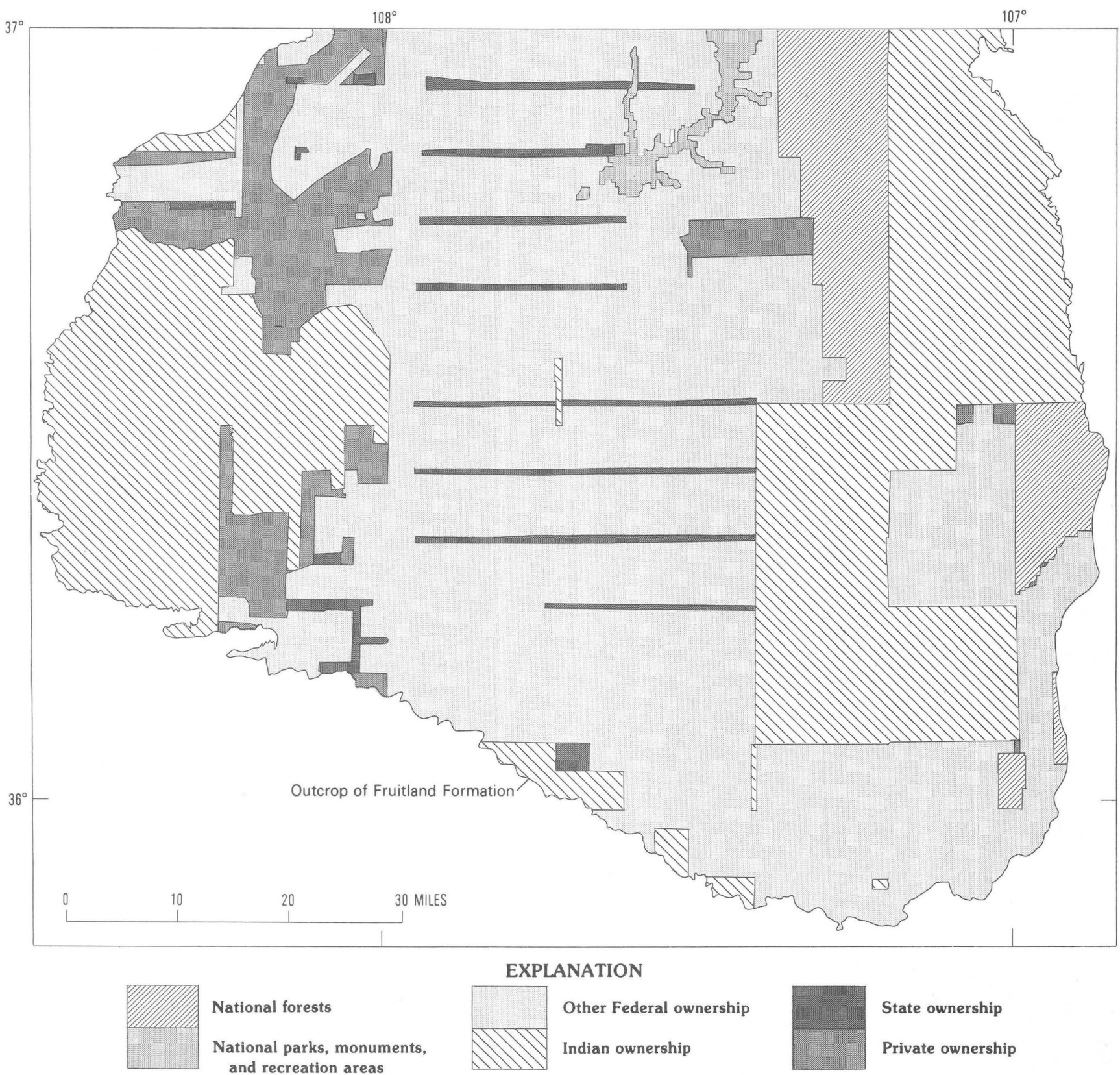

National forests
National parks, monuments,
and recreation areas

EXPLANATION

Figure B6. Digitized boundaries of land categories in area underlain by the Fruitland Formation. Boundaries are highly generalized and are intended to represent only the general proportions of each category in a given area. Ownership status of specific parcels of land should not be inferred from this map. See figure B1 for location of area shown here. 


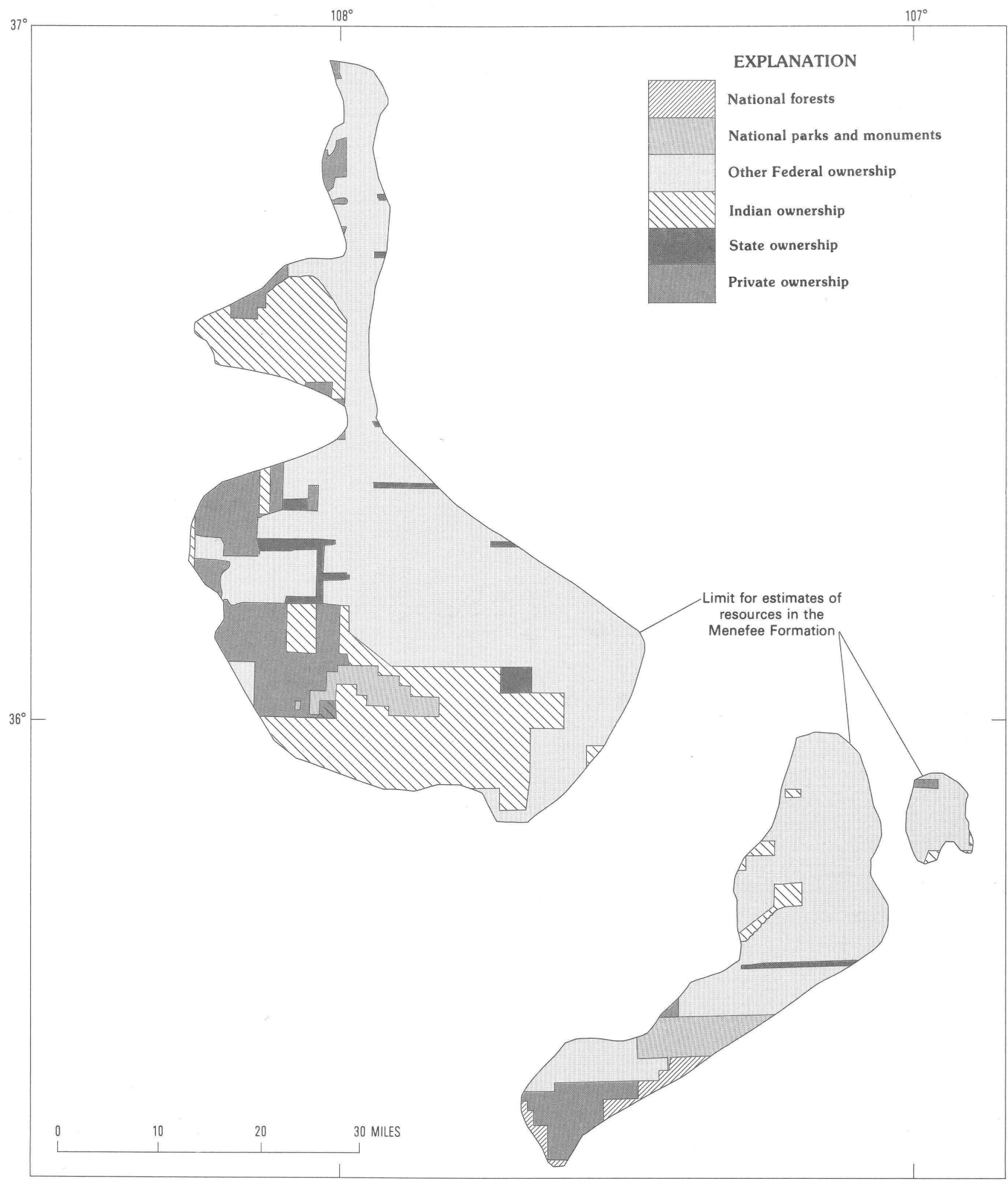

Figure B7. Digitized boundaries of land categories in areas underlain by the Menefee Formation. Boundaries are highly generalized and are intended to represent only the general proportions of each category in a given area; in certain cases, categories may be misapplied. Ownership status of specific parcels of land should not be inferred from this map. See figure B1 for location of area shown here. 
limit-of-estimate line generated by the geologists. This artificial boundary is used instead of an outcrop boundary to enclose the area best represented by the available data.

A small, isolated cluster of wells in the northeast portion of the basin also provided Menefee coal data. The coal in this small area is less than 5 feet in total thickness and is quite distant from the other Menefee coal to the south. No reliable resource calculation could be made for the small set of data from this isolated area.

\section{Resource Calculations}

The GARNET program uses a point location file, a gridded coal isopach file, and a boundary file to calculate the volume of coal, then the appropriate density factor for the rank of coal being considered is applied to produce a coal tonnage estimate. Coal resource volumes are calculated by determining the area (acreage) and average thickness for all of the grid cells of the coal isopach grid that fall within the area defined by the boundary file and a selected radius from the data point locations. The U.S. Geological Survey has designated specific radii from known coal data points as defining certain reliability classifications of coal resource tonnage estimates (Wood and others, 1983), but any radii can be selected and used in the GARNET program. These volumes are then multiplied by the density factor appropriate for the rank of the coal under consideration. For coal in the Menefee and Fruitland Formations, a density factor of 1,770 tons per acre foot was selected. ${ }^{1}$

The data included in NMSTRAT for the coal-bearing rocks older than the Fruitland and Menefee Formations (in descending order: Crevasse Canyon Formation, Gallup Sandstone, Tres Hermanos Formation, and Dakota Sandstone) are from localities scattered sparsely across the San Juan basin. Coal data in NMSTRAT are insufficient to allow use of computer methods to generate coal tonnage estimates for these rock units. The latest estimates by the New Mexico Bureau of Mines and Mineral Resources for the Crevasse Canyon Formation coal resources, shown below, were derived from coal data for the southern portion of the San Juan basin using a planimeter. Resource calculations done by conventional methods, using measured and indicated resource categories based on radii from known data points (Woods and others, 1983, p. 11), yielded the following estimate of coal in the Crevasse Canyon Formation, in beds more than 1.2 feet thick under less than 250 feet of overburden (Frank W. Campbell, New Mexico Bureau of Mines and Mineral Resources, oral commun., 1985):

\footnotetext{
${ }^{1}$ Minimum thicknesses specified by Wood and others (1983, table 3 ) were not used in classifying coal resources for these estimates. Coal quality data are insufficient to discriminate between bituminous and subbituminous coal. Resource estimates in this report represent total coal.
}

\begin{tabular}{cccc}
\hline \multicolumn{4}{c}{ CREVASSE CANYON FORMATION } \\
\hline $\begin{array}{c}\text { Resource } \\
\text { classification }\end{array}$ & Acres & $\begin{array}{c}\text { Average } \\
\text { thickness } \\
\text { (feet) }\end{array}$ & $\begin{array}{c}\text { Estimated } \\
\text { tons } \\
\text { (millions) }\end{array}$ \\
\hline $\begin{array}{l}\text { Measured ...... } \\
\text { Indicated ...... }\end{array}$ & 38,000 & 3.5 & 325 \\
\hline
\end{tabular}

\section{RESULTS}

The coal resources of the Fruitland and Menefee Formations were estimated by several different techniques for comparative purposes. The resource estimation techniques used were selected on the basis of data distribution, the accuracy and scale of boundary definitions, the size of area to be included, and research goals. All the estimated coal tonnages shown below are reported to the nearest million tons, which is deemed to be the maximum appropriate level of precision for this study.

Calculations using a single large radius from known data points, the Fruitland Formation outcrop, and the limit-of-estimate boundary of the Menefee Formation gave the following estimate of total coal resources:

\begin{tabular}{lccc}
\hline Formation & Acres & $\begin{array}{c}\text { Average } \\
\text { thickness } \\
\text { (feet) }\end{array}$ & $\begin{array}{c}\text { Estimated } \\
\text { tons } \\
\text { (millions) }\end{array}$ \\
\hline Fruitland & $3,720,299$ & 25.4 & 167,501 \\
Menefee & $1,216,901$ & 9.8 & 21,099 \\
\hline
\end{tabular}

Calculations using the radii specified by Wood and others (1983, fig. 4), the Fruitland Formation outcrop, and the limit-of-estimate boundary of the Menefee Formation gave this estimate of coal resources in the four reliability categories used by the USGS:

\begin{tabular}{lrrr}
\hline $\begin{array}{c}\text { Formation } \\
\text { and resource } \\
\text { classification }\end{array}$ & Acres & $\begin{array}{c}\text { Average } \\
\text { thickness } \\
\text { (feet) }\end{array}$ & $\begin{array}{c}\text { Estimated } \\
\text { tons } \\
\text { (millions) }\end{array}$ \\
\hline Fruitland: & & & \\
Measured .... & 71,225 & 23.8 & 3,001 \\
Indicated ..... & 400,686 & 23.7 & 16,774 \\
Inferred...... & $2,533,367$ & 26.1 & 116,980 \\
Hypothetical & 715,022 & 24.3 & 30,738 \\
& & & \\
Menefee: & 17,152 & 9.6 & 290 \\
Measured ..... & 143,997 & 8.7 & 2,229 \\
Indicated ...... & 835,090 & 9.8 & 14,438 \\
Inferred...... & 220,661 & 10.6 & 4,140 \\
Hypothetical & & & \\
\hline
\end{tabular}

The use of boundaries for some of the very generalized land ownership categories and a single large radius produced the following approximate total coal resource estimates: 


\begin{tabular}{|c|c|c|c|}
\hline $\begin{array}{l}\text { Ownership and } \\
\text { overburden } \\
\text { thickness (feet) }\end{array}$ & Acres & $\begin{array}{c}\text { Average } \\
\text { thickness } \\
\text { (feet) }\end{array}$ & $\begin{array}{c}\text { Estimated } \\
\text { tons } \\
\text { (millions) }\end{array}$ \\
\hline
\end{tabular}

Fruitland Formation

National forests:

\begin{tabular}{crrr}
$0-500$ & 11,894 & 0.1 & 1 \\
$500-1,000$ & 6,894 & 0.9 & 11 \\
$1,000-2,000$ & 19,068 & 2.1 & 72 \\
$2,000-3,000$ & 22,590 & 10.4 & 416 \\
$>3,000$ & 197,232 & 36.6 & 12,778 \\
\cline { 2 - 4 } Total & 257,678 & & 13,278 \\
\hline
\end{tabular}

National parks, monuments, and recreation areas:

$$
\begin{gathered}
2,000-3,000 \\
>3,000
\end{gathered}
$$

Total

\begin{tabular}{rrr}
38,360 & 46.7 & 3,170 \\
7,728 & 53.7 & 735 \\
\hline 46,088 & & 3,905 \\
\hline
\end{tabular}

Other federal ownership:

$$
\begin{gathered}
0-500 \\
500-1,000 \\
1,000-2,000 \\
2,000-3,000 \\
>3,000 \\
\text { Total }
\end{gathered}
$$

\begin{tabular}{rrr}
343,165 & 14.0 & 8,842 \\
227,083 & 12.8 & 5,284 \\
553,550 & 14.5 & 13,352 \\
601,801 & 33.2 & 36,480 \\
323,498 & 47.4 & 26,498 \\
\hline $2,049,097$ & & 90,456 \\
\hline
\end{tabular}

Indian lands:

\begin{tabular}{|c|c|c|c|}
\hline $\begin{array}{l}\text { Ownership and } \\
\text { overburden } \\
\text { thickness (feet) }\end{array}$ & Acres & $\begin{array}{c}\text { Average } \\
\text { thickness } \\
\text { (feet) }\end{array}$ & $\begin{array}{c}\text { Estimated } \\
\text { tons } \\
\text { (millions) }\end{array}$ \\
\hline \multicolumn{4}{|c|}{ Menefee Formation-Continued } \\
\hline $\begin{array}{c}\text { Indian lands: } \\
0-500 \\
500-1,000 \\
1,000-2,000 \\
2,000-3,000 \\
>3,000\end{array}$ & $\begin{array}{r}166,273 \\
8,745 \\
309 \\
49,952 \\
22,492\end{array}$ & $\begin{array}{r}11.4 \\
7.1 \\
9.2 \\
4.2 \\
7.6\end{array}$ & $\begin{array}{r}3,345 \\
109 \\
5 \\
370 \\
303\end{array}$ \\
\hline Total & 247,771 & & 4,132 \\
\hline $\begin{array}{l}\text { State lands } \\
\text { unclassified: }\end{array}$ & 14,456 & 16.3 & 416 \\
\hline
\end{tabular}

$$
\begin{gathered}
0-500 \\
500-1,000 \\
1,000-2,000 \\
2,000-3,000 \\
>3,000 \\
\text { Total }
\end{gathered}
$$

\begin{tabular}{lll}
209,267 & 20.1 & 7,450 \\
139,483 & 18.0 & 4,435 \\
328,728 & 14.2 & 8,282 \\
245,185 & 16.4 & 7,115 \\
211,296 & 26.6 & 9,939 \\
\hline $1,133,959$ & & 37,221 \\
\hline
\end{tabular}

\section{State lands} unclassified:

46,700

37.9

3,129
Detailed resource estimates for the "Other Federal ownership" category, classified by overburden, county, and township and range, are given in tables B1 and B2 at the end of the chapter.

A comparison of the total coal resources tonnage estimate calculated by the computer and the summation of resource numbers for the various multiple resource calculations shows some differences:

\begin{tabular}{ccc}
\hline \multirow{2}{*}{ Method of calculation } & $\begin{array}{c}\text { Estimated total coal resources } \\
\text { (millions of tons) }\end{array}$ \\
\cline { 2 - 3 } & $\begin{array}{c}\text { Fruitland } \\
\text { Formation }\end{array}$ & $\begin{array}{c}\text { Menefee } \\
\text { Formation }\end{array}$ \\
\hline Based on a single large radius.... & 167,501 & 21,099 \\
$\begin{array}{c}\text { Total of four reliability categories } \\
\text { of Wood and others }(1983) \ldots . . .\end{array}$ & 167,493 & 21,097 \\
$\begin{array}{c}\text { Total of estimates for various land } \\
\text { ownership categories ............ }\end{array}$ & 147,989 & 15,944 \\
\hline
\end{tabular}

The significant "shortfall" in the estimates tallied from the land ownership categories results mainly from the exclusion of privately owned resources from these estimates. Other, smaller differences in the total resource estimates are mostly attributable to three factors:

1. The size of the area being estimated (estimates for small areas have a greater margin of error than those for large areas).

2. The grid cell interval.

3. The GARNET resource calculation procedure that includes or excludes all of a particular grid cell depending on its intersection with a boundary. 


\section{CONSIDERATIONS AND ISSUES}

The variability in number of coal beds across the basin (fig. B8) demonstrates the discontinuous nature of coal beds within the Fruitland Formation coal zone. A comparison of figure B8 with figure B2 will indicate the variability in the number of coal beds in which a given total coal thickness occurs. This information can be used, along with interburden data, to define areas most favorable for development.

Several refinements would improve the utility of the results of these resource calculations. Some of these could be accomplished fairly soon:

1. Improving acreage and tonnage estimates, especially with reference to federal ownership, by using digital boundary data recorded from 1:100,000-scale or larger scale maps.
2. Calculating resources by thickness category of individual coal horizons; that is, summing coal horizons whose thicknesses fall within a given range, such as $10-20$ feet or 20-30 feet. These resource numbers categorized according to overburden thickness would reflect information of value for potential development.

Other, longer range improvements would require additional data gathering and study:

1. Expansion and refinement of the stratigraphic data base; location and encoding of additional coal stratigraphic data.

2. Further review and analyses of correlations within the data base.

3. Gathering and encoding sufficient data on coal quality to allow resource estimates to be subdivided into coal quality categories.

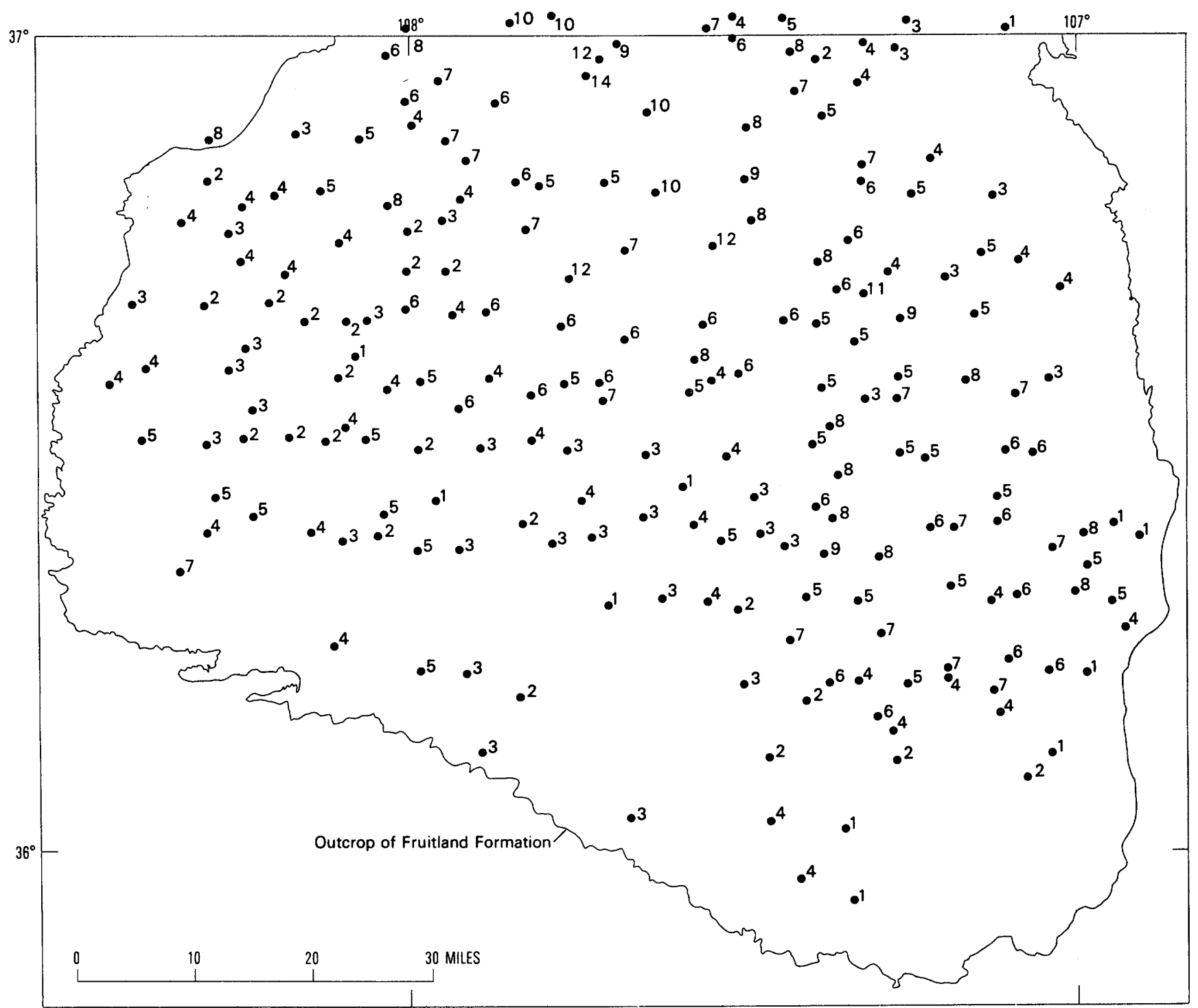

Figure B8. Number of coal horizons in the Fruitland Formation at drill hole localities. See figure B1 for location of area shown here. 
Table B1. Estimates of Federally owned subbituminous coal resources in the Fruitland Formation, San Juan basin, New Mexico

[Estimates may include some coal already mined but exclude resources in national parks, monuments, and national forests]

\begin{tabular}{|c|c|c|c|}
\hline \multirow{2}{*}{$\begin{array}{l}\text { Location and } \\
\text { overburden } \\
\text { thickness } \\
\text { (feet) }\end{array}$} & \multirow{2}{*}{ Acres } & \multirow{2}{*}{$\begin{array}{c}\text { Average } \\
\text { total } \\
\text { thick- } \\
\text { ness } \\
\text { (feet) }\end{array}$} & $\begin{array}{l}\text { Millions of } \\
\text { short tons }\end{array}$ \\
\hline & & & $\begin{array}{c}\text { Each } \\
\text { interval }\end{array}$ \\
\hline
\end{tabular}

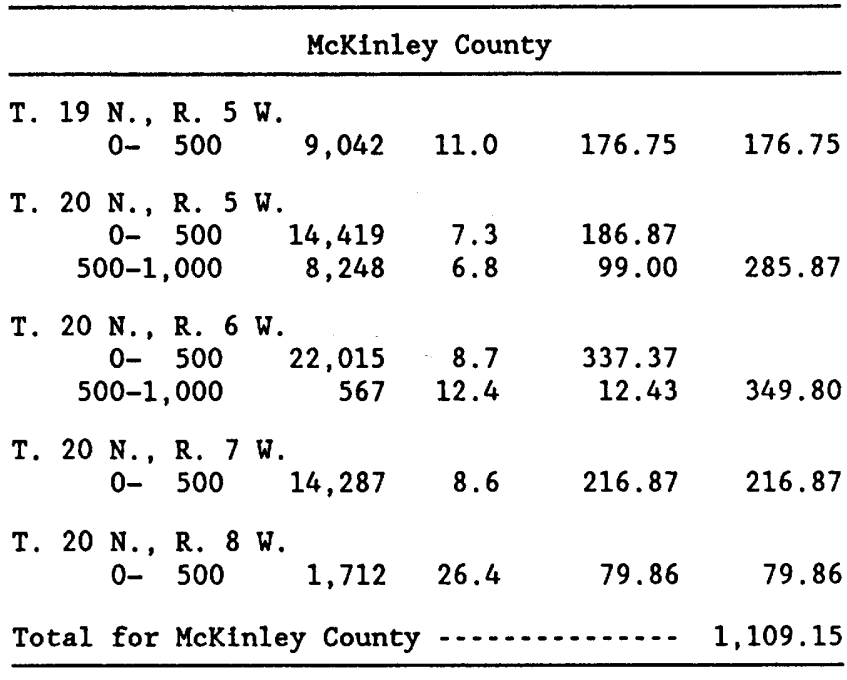

Rio Arriba County

\section{T. 23 N., R. 1 W.}

$\begin{array}{rrrr}0-500 & 485 & .3 & .23 \\ 500-1,000 & 1,163 & 2.6 & 5.39 \\ 1,000-2,000 & 2,291 & 4.2 & 16.82 \\ 2,000-3,000 & 4,199 & 9.5 & 70.93\end{array}$

\section{T. $23 \mathrm{~N} .$, R. 6 W.}

$$
1,000-2,000
$$

T. $23 \mathrm{~N} ., \mathrm{R} .7 \mathrm{~W}$. $1,000-2,000$
$2,000-3,000$

\section{9,807}

710

\section{2}

159.62

T. 24 N., R. 1 E.

$$
0-500
$$

500-1,000

$$
2,797
$$

195

\section{.5}

$$
2.69
$$

T. 24 N., R. 1 W.

$$
\begin{array}{r}
1,000-2,000 \\
2,000-3,000 \\
>3,000
\end{array}
$$

5,348
13,762
1,535

10.9

17.2

36.7

103.35

418.02

99.77

T. 24 N., R. 2 W.

$$
\begin{array}{r}
2,000-3,000 \\
>3,000
\end{array}
$$

$$
5,721
$$

17.6

178.07

17,056

24.5

740.21

T. 24 N., R. 3 W.

$$
\begin{array}{r}
2,000-3,000 \\
>3,000
\end{array}
$$

$$
6,858
$$

22.0

266.86 13,977

25.8

637.02

T. 24 N., R. 6 W.

$$
1,000-2,000
$$

$$
17,018
$$

13.2

398.42

2,000-3,000

$$
4,753
$$

14.2

119.21

T. 24 N., R. 7 W.
$1,000-2,000$
2,740
8.4
40.49
$2,000-3,000$
16,300
374.45

414.94
93.37

239.37

172.14

621.14

918.28

903.88

517.63
Table B1. Estimates of Federally owned subbituminous coal resources in the Fruitland Formation, San Juan basin, New Mexico-Continued

\begin{tabular}{lccc}
\hline $\begin{array}{c}\text { Location and } \\
\text { overburden } \\
\text { thickness } \\
\text { (feet) }\end{array}$ & Acres & $\begin{array}{c}\text { Average } \\
\text { total } \\
\text { thick- } \\
\text { ness } \\
\text { (feet) }\end{array}$ & $\begin{array}{c}\text { Millions of } \\
\text { short tons }\end{array}$ \\
\hline & Rinterval & Total \\
\hline
\end{tabular}

T. 25 N., R. 1 E.

$\begin{array}{crrrr}500-1,000 & 1,604 & 1.8 & 5.06 & \\ 1,000-2,000 & 1,091 & 2.2 & 4.24 & 9.30 \\ 25 \text { N., R. 2 W. } & & & & \\ >3,000 & 23,975 & 16.7 & 708.20 & 708.20 \\ 25 \text { N., R. 3 W. } & & & & \\ >3,000 & 22,751 & 30.3 & 1,218.81 & 1,218.81\end{array}$

T. 25 N., R. 6 W. $2,000-3,000 \quad 21,276 \quad 14.6 \quad 549.68 \quad 549.68$

T. 25 N., R. 7 W. $\begin{array}{rrrr}1,000-2,000 & 571 & 12.6 & 12.73\end{array}$

$\begin{array}{lllll}2,000-3,000 & 18,085 & 16.6 & 529.85 & 542.58\end{array}$

T. 26 N., R. 2 W. $>3,000 \quad 19,435 \quad 18.9 \quad 650.74 \quad 650.74$

T. 26 N., R. 6 W. $\begin{array}{lllll}2,000-3,000 & 21,677 & 20.9 & 802.87 & 802.87\end{array}$

T. $26 \mathrm{~N} .$, R. 7 W. $2,000-3,000 \quad 18,763 \quad 24.5 \quad 811.91 \quad 811.91$

T. 27 N., R. 5 W. $>3,000 \quad 22,629$

$47.2 \quad 1,892.03 \quad 1,892.03$

T. 27 N., R. 6 W. $2,000-3,000 \quad 9,229 \quad 40.2 \quad 656.10$ $>3,000 \quad 10,985$

50.7

$986.45 \quad 1,642.55$

T. 27 N., R. 7 W. $2,000-3,000 \quad 18,811$

$40.6 \quad 1,351.17 \quad 1,351.17$

T. 28 N., R. 5 W. $>3,000 \quad 17.337$

$59.6 \quad 1,827.52 \quad 1,827.52$

T. $28 \mathrm{~N} .$, R. $6 \mathrm{~W}$. $2,000-3,000 \quad 1,720$

$60.6 \quad 184.32$ $>3,000 \quad 13,636$

$56.5 \quad 1,362.54 \quad 1,546.86$

T. $28 \mathrm{~N} .$, R. $7 \mathrm{~W}$. $2,000-3,000 \quad 17,106 \quad 54.8 \quad 1,657.97 \quad 1,657.97$

T. 29 N., R. 5 W. $>3,000$

T. 29 N., R. 6 W. $>3,000 \quad 10,450$

63.6

902.29

902.29

T. 29 N., R. 7 W.

$2,000-3,000$ $>3,000 \quad 17,607$

$60.2 \quad 1,113.41 \quad 1,113.41$

T. 30 N., R. 5 W. $>3,000 \quad 13,518$

$77.3 \quad 449.53$

$75.32,348.00$

$2,797.53$

T. 30 N., R. $6 \mathrm{~W}$. $\begin{array}{rrrr}2,000-3,000 & 557 & 77.7 & 76.59 \\ >3,000 & 20,789 & 55.1 & 2,027.38\end{array}$

$2,027.38 \quad 2,103.97$ 
Table B1. Estimates of Federally owned subbituminous coal resources in the Fruitland Formation, San Juan basin, New Mexico-Continued

\begin{tabular}{lccc}
\hline $\begin{array}{l}\text { Location and } \\
\text { overburden } \\
\text { thickness } \\
\text { (feet) }\end{array}$ & Acres & $\begin{array}{c}\text { Average } \\
\text { total } \\
\text { thick- } \\
\text { ness } \\
\text { (feet) }\end{array}$ & $\begin{array}{c}\text { Millions of } \\
\text { short tons }\end{array}$ \\
\hline
\end{tabular}

Rio Arriba County--Continued

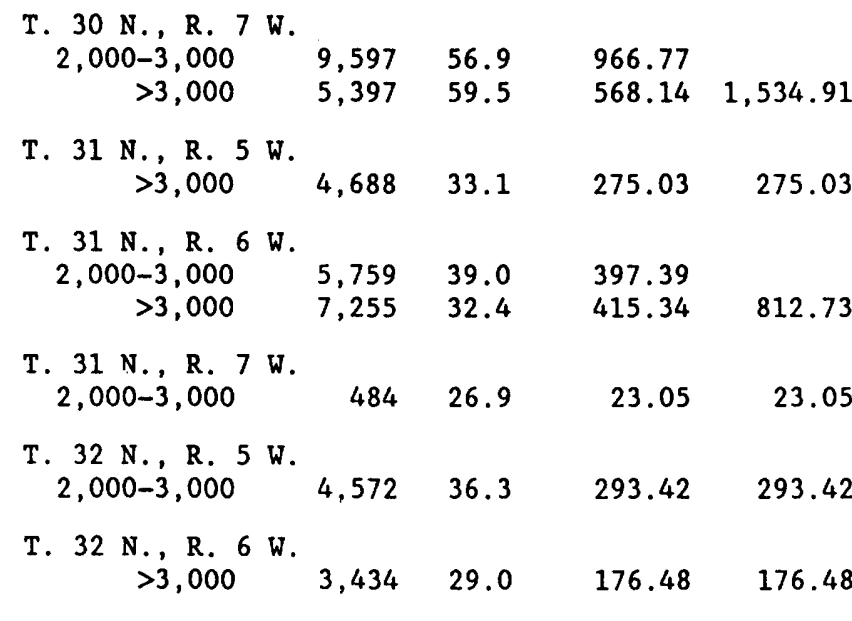

Total for Rio Arriba County ......... . . 30,292.45

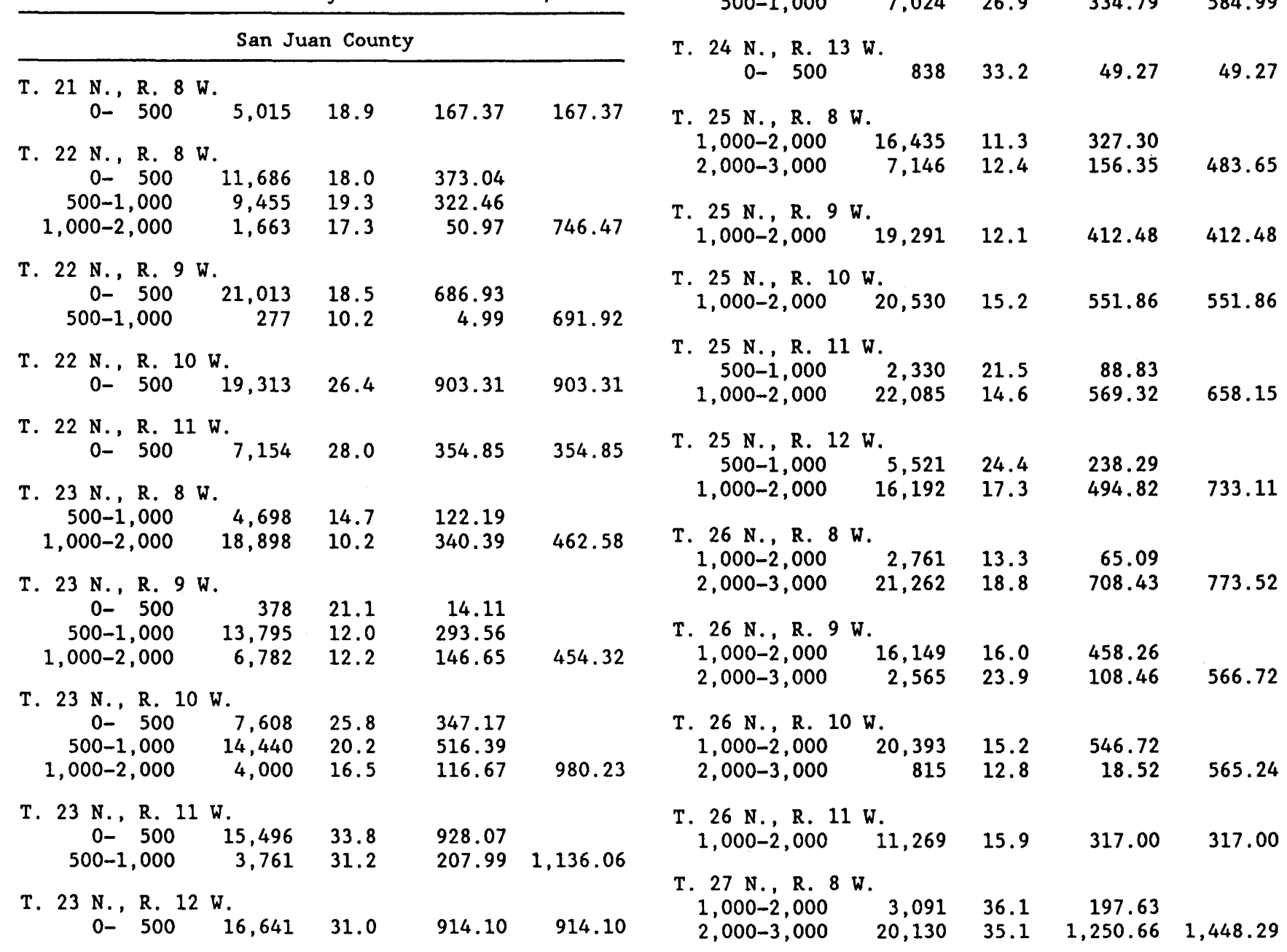

Table B1. Estimates of Federally owned subbituminous coal resources in the Fruitland Formation, San Juan basin, New Mexico-Continued

\begin{tabular}{|c|c|c|c|c|}
\hline \multirow{2}{*}{$\begin{array}{l}\text { Location and } \\
\text { overburden } \\
\text { thickness } \\
\text { (feet) }\end{array}$} & \multirow{2}{*}{ Acres } & \multirow{2}{*}{$\begin{array}{c}\text { Average } \\
\text { total } \\
\text { thick- } \\
\text { ness } \\
\text { (feet) }\end{array}$} & \multicolumn{2}{|c|}{$\begin{array}{l}\text { Millions of } \\
\text { short tons }\end{array}$} \\
\hline & & & $\begin{array}{c}\text { Each } \\
\text { interval }\end{array}$ & Total \\
\hline
\end{tabular}

San Juan County--Continued

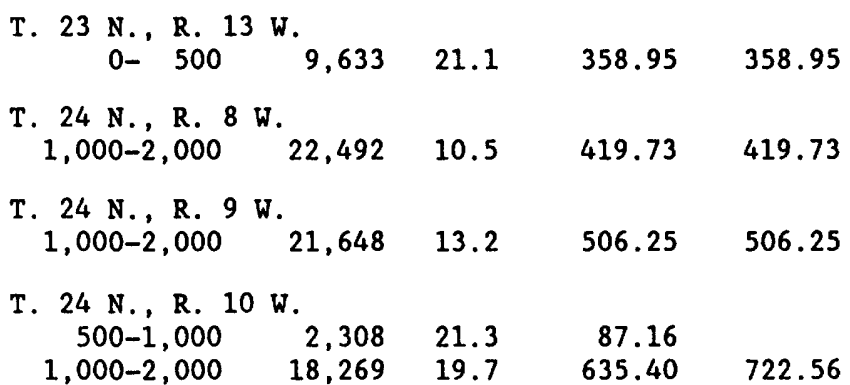

T. 24 N., R. 11 W.

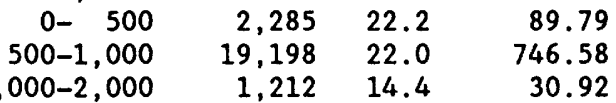

867.29

T. 24 N., R. 12 W.

$\begin{array}{cccc}0-500 & 4,249 & 33.3 & 250.2 \\ 500-1,000 & 7.024 & 26.9 & 334.7\end{array}$

84.99

9.27 
Table B1. Estimates of Federally owned subbituminous coal resources in the Fruitland Formation, San Juan basin, New Mexico-Continued

\begin{tabular}{|c|c|c|c|}
\hline $\begin{array}{l}\text { Location and } \\
\text { overburden }\end{array}$ & & $\begin{array}{c}\text { Average } \\
\text { total }\end{array}$ & $\begin{array}{l}\text { Millions of } \\
\text { short tons }\end{array}$ \\
\hline $\begin{array}{l}\text { thickness } \\
\text { (feet) }\end{array}$ & & $\begin{array}{l}\text { ness } \\
\text { (feet) }\end{array}$ & $\begin{array}{c}\text { Each } \\
\text { Interval }\end{array}$ \\
\hline
\end{tabular}

San Juan County--Continued

T. 27 N., R. 9 W.

$\begin{array}{llll}1,000-2,000 & 1,686 & 41.5 & 123.75\end{array}$

$\begin{array}{lllll}2,000-3,000 & 16,554 & 36.3 & 1,062.33 & 1,186.08\end{array}$

T. 27 N., R. 10 W.

$\begin{array}{llll}1,000-2,000 & 15,983 & 29.0 & 820.87\end{array}$

$\begin{array}{lllll}2,000-3,000 & 5,014 & 25.3 & 224.68 & 1,045.55\end{array}$

T. 27 N., R. 11 W.

$1,000-2,000 \quad 11,984 \quad 20.1 \quad 427.14 \quad 427.14$

T. $28 \mathrm{~N} .$, R. 8 W.

$\begin{array}{lllll}2,000-3,000 & 17,661 & 48.7 & 1,522.29 & 1,522.29\end{array}$

T. $28 \mathrm{~N},$, R. 9 W.

$2,000-3,000 \quad 17,699$

$40.1 \quad 1,256.76 \quad 1,256.76$

T. 28 N., R. $10 \mathrm{~W}$.

$\begin{array}{lllll}1,000-2,000 & 9,702 & 30.2 & 517.84 & \\ 2,000-3,000 & 8,065 & 32.0 & 456.50 & 974.34\end{array}$

T. 28 N., R. $11 \mathrm{~W}$.

$\begin{array}{lllll}1,000-2,000 & 16,244 & 18.4 & 528.81 & 528.81\end{array}$

T. $28 \mathrm{~N} .$, R. 12 W.

$1,000-2,000 \quad 3,580$

T. $28 \mathrm{~N} .$, R. $13 \mathrm{w}$.

$\begin{array}{lllll}1,000-2,000 & 1,275 & 14.1 & 31.83 & 31.83\end{array}$

T. 29 N., R. 8 W.

$\begin{array}{lllll}2,000-3,000 & 20,538 & 60.8 & 2,210.17 & 2,210.17\end{array}$

T. 29 N., R. 9 W.

$2,000-3,000 \quad 20,436 \quad 41.4 \quad 1,498.00 \quad 1,498.00$

T. 29 N., R. $10 \mathrm{~W}$.

$\begin{array}{llll}1,000-2,000 & 7,926 & 25.2 & 353.65\end{array}$

$\begin{array}{lllll}2,000-3,000 & 9,602 & 26.6 & 452.43 & 806.08\end{array}$

T. 29 N., R. 11 W.

$\begin{array}{lllll}1,000-2,000 & 17,664 & 15.3 & 477.78 & 477.78\end{array}$

T. 29 N., R. 12 W.

$1,000-2,000 \quad 4,453$

$18.4 \quad 144.99 \quad 144.99$

T. 29 N., R. $13 \mathrm{~W}$.

$1,000-2,000 \quad 2,849$

T. $30 \mathrm{~N},$, R. $8 \mathrm{~W}$.

$\begin{array}{rrrrr}2,000-3,000 & 15,717 & 57.9 & 1,611.04 & \\ >3,000 & 765 & 61.9 & 83.78 & 1,694.82\end{array}$

T. 30 N., R. 9 W.

$\begin{array}{lllll}2,000-3,000 & 18,945 & 39.7 & 1,329.51 & 1,329.51\end{array}$

T. 30 N., R. $10 \mathrm{w}$.

$\begin{array}{lllll}2,000-3,000 & 19,547 & 31.2 & 1,079.20 & 1,079.20\end{array}$

T. 30 N., R. 11 W.

$\begin{array}{rrrrr}1,000-2,000 & 5,402 & 20.2 & 192.63 & \\ 2,000-3,000 & 12,024 & 30.7 & 653.99 & 846.62\end{array}$

Table B1. Estimates of Federally owned subbituminous coal resources in the Fruitland Formation, San Juan basin, New Mexico-Continued

\begin{tabular}{|c|c|c|c|}
\hline $\begin{array}{l}\text { Location and } \\
\text { overburden }\end{array}$ & & $\begin{array}{c}\text { Average } \\
\text { total }\end{array}$ & $\begin{array}{l}\text { Milifons of } \\
\text { short tons }\end{array}$ \\
\hline $\begin{array}{l}\text { thickness } \\
\text { (feet) }\end{array}$ & 20100 & $\begin{array}{l}\text { ness } \\
\text { (feet) }\end{array}$ & $\begin{array}{c}\text { Each } \\
\text { Interval }\end{array}$ \\
\hline
\end{tabular}

San Juan County--Continued

T. 30 N., R. 12 W.

$1,000-2,000$

$2,000-3,000$

3,747

3,747

20.6

21.9

136.76

91.54

228.30

T. $30 \mathrm{~N} .$, R. $13 \mathrm{~W}$.

500-1,000

$1,000-2,000$

291

13.8

21.6

7.13

294.49

301.62

T. 30 N., R. 14 W.

$0-500$
$500-1,000$
$1,000-2,000$

401

16.2

20.6

11.48

428.24

62.13

501.85

T. 30 N., R. 15 W.

$\begin{array}{rrrr}0-500 & 9,030 & 17.4 & 277.57 \\ 500-1,000 & 692 & 28.6 & 34.94\end{array}$

312.51

T. 31 N., R. 6 W.

$2,000-3,000$

3,659

43.4

281.31

281.31

T. $31 \mathrm{~N} .$, R. $7 \mathrm{~W}$. $\begin{array}{rr}2,000-3,000 & 13,394 \\ >3,000 & 1,641\end{array}$

$\begin{array}{rrr}47.8 & 1,132.53 & \\ 43.9 & 127.64 & 1,260.17\end{array}$

T. 31 N., R. 8 W.

$2,000-3,000$
$>3,000$

2,273

17,299

71.0

285.57

59.2

$1,811.78$

$2,097.35$

T. 31 N., R. 9 W.

$$
\begin{array}{rr}
2,000-3,000 & 17,512 \\
>3,000 & 526
\end{array}
$$

$49.3 \quad 1,527.82$

526

61.8

$57.58 \quad 1,585.40$

T. 31 N., R. $10 \mathrm{w}$.

$2,000-3,000 \quad 20,306$

$39.3 \quad 1,413.61 \quad 1,413.61$

T. 31 N., R. 11 W.

$2,000-3,000 \quad 22,382$

35.9

$1,421.46 \quad 1,421.46$

T. 31 N., R. 12 W. $2,000-3,000 \quad 22,510$

31.1

$1,237.96 \quad 1,237.96$

T. 31 N., R. $13 \mathrm{~W}$. 500-1,000

$1,000-2,000$

2,293

$2,000-3,000$

1,618

16.5
31.8

66.87

90.93

$31.6 \quad 190.01$

347.81

T. 32 N., R. 6 W.

$2,000-3,000$

9,654

40.5

691.72

691.72

T. 32 N., R. 7 W.

2,000-3,000

204

45.0

16.27

16.27

T. 32 N., R. 8 W.

$$
>3,000 \quad 15,045
$$

58.8

$1,565.95 \quad 1,565.95$

T. 32 N., R. 9 W. $>3,000 \quad 13,297$

54.9

$1,292.39 \quad 1,292.39$

T. 32 N., R. $10 \mathrm{~W}$. $\begin{array}{rr}2,000-3,000 & 7,811 \\ >3,000 & 20,455\end{array}$

$56.7 \quad 783.60$

$\begin{array}{lll}67.5 & 2,445.28 & 3,228.88\end{array}$

30 Coalfields of New Mexico: Geology and Resources 
Table B1. Estimates of Federally owned subbituminous coal resources in the Fruitland Formation, San Juan basin, New Mexico-Continued

\begin{tabular}{|c|c|c|c|c|}
\hline \multirow{2}{*}{$\begin{array}{l}\text { Location and } \\
\text { overburden } \\
\text { thickness } \\
\text { (feet) }\end{array}$} & \multirow{2}{*}{ Acres } & \multirow{2}{*}{$\begin{array}{c}\text { Average } \\
\text { total } \\
\text { thick- } \\
\text { ness } \\
\text { (feet) }\end{array}$} & \multicolumn{2}{|c|}{$\begin{array}{l}\text { Millions of } \\
\text { short tons }\end{array}$} \\
\hline & & & $\begin{array}{c}\text { Each } \\
\text { interval }\end{array}$ & Total \\
\hline
\end{tabular}

San Juan County--Continued

T. 32 N., R. $11 \mathrm{~W}$.

$2,000-3,000 \quad 13,086 \quad 48.8 \quad 1,130.57 \quad 1,130.57$

T. $32 \mathrm{~N} .$, R. $12 \mathrm{~W}$.

$2,000-3,000 \quad 2,298 \quad 40.9 \quad 166.44 \quad 166.44$

T. 32 N., R. 13 W.

$\begin{array}{lllll}500-1,000 & 3,029 & 34.3 & 183.74 & \\ , 000-2,000 & 2,197 & 42.3 & 163.96 & 347.70\end{array}$

Total for San Juan County ........... 53,514.82

Sandoval County

T. 17 N., R. 2 W.

$\begin{array}{llllll}0- & 500 & 8,140 & 3.3 & 47.79 & 47.79\end{array}$

T. 17 N., R. 3 W.

O- $\begin{array}{lllll}500 & 21,631 & 7.6 & 291.22 & 291.22\end{array}$

T. 17 N., R. 4 W.

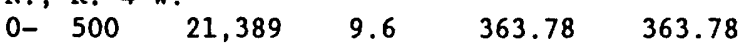

T. $18 \mathrm{~N} ., \mathrm{R} .1 \mathrm{~W}$.

$\begin{array}{lllll}0-500 & 3,028 & 3.3 & 17.71 & 17.71\end{array}$

T. 18 N., R. 2 W. 0 - $500 \quad 7,651$

$5.6 \quad 75.38$

75.38

T. $18 \mathrm{~N} ., \mathrm{R}, 3 \mathrm{~W}$ $\begin{array}{llll}0-500 & 22,567 & 14.6 & 584.03\end{array}$ $\begin{array}{rrrr}500-1,000 & 125 & 4.2 & .92\end{array}$

T. 18 N., R. $4 \mathrm{~W}$. 500-1,000 1,898

8.5

28.46

584.95

T. 19 N., R. 2 W. $0-500$

T. 19 N., R. $3 \mathrm{~W}$. $0-500$

T. 19 N., R. 4 W. $0-500 \quad 15,010$

130.25

T. 20 N., R. 1 W. 500-1,000 $1,000-2,000$ 336 764

.7

.39

$2.9 \quad 3.96$

4.35

T. 20 N., R. 2 W. $0-500$ $500-1,000$ 4, 405

1.3

10.24 $1,000-2,000$ 15,503

$2,000-3,000$

12,408

.7

19.30

$5.3 \quad 12.24$

$\begin{array}{ll}6.6 & 144.57\end{array}$

T. 20 N., R. 3 W.

$$
\text { O- } 500
$$

500-1,000

$$
8,150
$$

10.3

148.40

$1,000-2,000$

12,880

8.6

196.72

89.15

28.46

10.44

54.57

130.25

T. 20 N., R. 4 W.

$$
0-500 \quad 11,594
$$

500-1,000

11,089

$10.3 \quad 211.56$ $9.1 \quad 178.50$

434.27

390.06
Table B1. Estimates of Federally owned subbituminous coal resources in the Fruitland Formation, San Juan basin, New Mexico-Continued

\begin{tabular}{lccc}
\hline $\begin{array}{l}\text { Location and } \\
\text { overburden } \\
\text { thickness } \\
\text { (feet) }\end{array}$ & Acres & $\begin{array}{c}\text { Average } \\
\text { total } \\
\text { thick- } \\
\text { ness } \\
\text { (feet) }\end{array}$ & $\begin{array}{c}\text { Millions of } \\
\text { short tons }\end{array}$ \\
\hline
\end{tabular}

\begin{tabular}{|c|c|c|}
\hline $\begin{array}{cr}0- & 500 \\
500-1,000 \\
000-2,000\end{array}$ & $\begin{array}{l}4,751 \\
6,917 \\
1,726\end{array}$ & $\begin{array}{r}1.0 \\
1.6 \\
.6\end{array}$ \\
\hline
\end{tabular}

Sandoval County--Continued

T. 21 N., R. 1 W.

29.75

T. 21 N., R. 2 W.

$\begin{array}{rrrr}1,000-2,000 & 20,721 & 2.5 & 92.38 \\ 2,000-3,000 & 1,777 & 5.0 & 15.69\end{array}$

108.07

T. 21 N., R. 3 w.

$\begin{array}{rrrr}1,000-2,000 & 15,955 & 6.2 & 176.27 \\ 2,000-3,000 & 6,885 & 12.4 & 150.80\end{array}$

327.07

T. 21 N., R. 4 W.

$\begin{array}{llll}500-1,000 & 16,913 & 4.7 & 140.72\end{array}$

$\begin{array}{llll}1,000-2,000 & 5,466 & 3.2 & 30.43\end{array}$

171.15

T. 21 N., R. 5 W. $\begin{array}{llll}500-1,000 & 9,860 & 8.1 & 141.88\end{array}$

$\begin{array}{llll}1,000-2,000 & 12,904 & 6.9 & 157.33\end{array}$

299.21

T. 21 N., R. 6 W. $\begin{array}{llll}500-1,000 & 17,406 & 12.6 & 389.24\end{array}$ $\begin{array}{rrrr}1,000-2,000 & 2,810 & 11.4 & 56.78\end{array}$

446.02

T. 21 N., R. 7 W. $\begin{array}{lllll}0- & 500 & 7,224 & 12.6 & 160.80\end{array}$ $\begin{array}{llll}500-1,000 & 8,672 & 11.2 & 171.21\end{array}$ $\begin{array}{llll}1,000-2,000 & 6,828 & 9.4 & 114.08\end{array}$

446.09

T. 22 N., R. 1 W.

$\begin{array}{rrrr}500-1,000 & 2,918 & 1.0 & 4.96 \\ 1,000-2,000 & 5,541 & 2.1 & 20.32 \\ 2,000-3,000 & 1,582 & 1.3 & 3.68\end{array}$

28.96

T. 22 N., R. 6 W.

$\begin{array}{llll}1,000-2,000 & 22,888 & 7.2 & 292.82\end{array}$

292.82

T. 22 N., R. 7 W.

$\begin{array}{llll}500-1,000 & 8,589 & 11.9 & 181.40\end{array}$

$\begin{array}{rrrr}1,000-2,000 & 14,285 & 9.4 & 238.53\end{array}$

419.93

T. 23 N., R. 1 W.

$\begin{array}{rrrr}0-500 & 359 & .4 & .22 \\ 500-1,000 & 1,337 & 2.1 & 4.95 \\ , 000-2,000 & 2,688 & 4.2 & 19.89 \\ , 000-3,000 & 1,351 & 2.1 & 4.98\end{array}$

30.04

T. $23 \mathrm{~N} .$, R. 6 W.

$\begin{array}{lllll}1,000-2,000 & 12,103 & 6.9 & 147.89 & 147.89\end{array}$

T. 23 N., R. 7 W.

$\begin{array}{lllll}1,000-2,000 & 10,002 & 9.8 & 173.00 & 173.00\end{array}$

Total for Sandoval County $\ldots \ldots \ldots \ldots \ldots \ldots . \ldots 5,539.58$ 
Table B2. Estimates of Federally owned subbituminous coal resources in the Menefee Formation, San Juan basin, New Mexico

[Estimates may include some coal already mined but exclude resources in national parks, monuments, and national forests]

\begin{tabular}{lccc}
\hline $\begin{array}{l}\text { Location and } \\
\text { overburden } \\
\text { thickness } \\
\text { (feet) }\end{array}$ & Acres & $\begin{array}{c}\text { Average } \\
\text { total } \\
\text { thick- } \\
\text { ness }\end{array}$ & $\begin{array}{c}\text { Millions of } \\
\text { short tons }\end{array}$ \\
\hline
\end{tabular}

McKinley County

T. $15 \mathrm{~N} ., \mathrm{R}, 6 \mathrm{~W}$.

$$
\begin{array}{llllll}
0- & 500 & 9,175 & 8.5 & 137.69 & 137.69
\end{array}
$$

T. 15 N., R. 7 W.

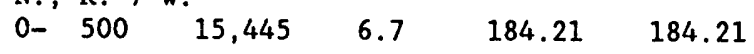

T. $15 \mathrm{~N},$, R. $8 \mathrm{~W}$.

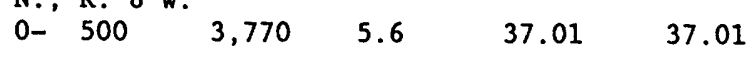

T. 16 N., R. 5 W.

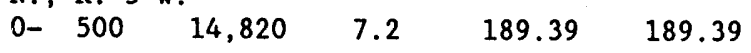

T. 19 N., R. 7 W. $\begin{array}{lllll}500-1,000 & 607 & 7.9 & 8.47 & 8.47\end{array}$

T. $19 \mathrm{~N} .$, R. $8 \mathrm{~W}$. $0-\quad 500 \quad 3,115 \quad 5.8 \quad 31.91$ $\begin{array}{llll}500-1,000 & 3,794 & 7.8 & 52.12\end{array}$

84.03

T. 19 N., R. 9 W. $0-500 \quad 1,470 \quad 4.0 \quad 10.39 \quad 10.39$

T. 20 N., R. 7 W. $0-\quad 500 \quad 9,963 \quad 16.0 \quad 281.41$ $\begin{array}{rrrr}500-1,000 & 3,414 & 8.1 & 48.61\end{array}$

330.02

T. $20 \mathrm{~N}$, , R. $8 \mathrm{~W}$. $\begin{array}{llll}0-500 & 4,895 & 10.3 & 89.03\end{array}$ $\begin{array}{llll}500-1,000 & 6,477 & 7.6 & 86.67\end{array}$

175.70

Total for McKinley County $\ldots \ldots \ldots \ldots \ldots \ldots 1$

San Juan County

T. 21 N., R. 8 W.

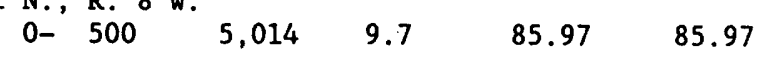

T. 21 N., R. $13 \mathrm{~W}$. $\begin{array}{lllll}0-500 & 3,074 & 2.4 & 13.25 & 13.25\end{array}$

T. 22 N., R. 8 W. $\begin{array}{lllll}500 & 22,943 & 15.3 & 620.63 & 620.63\end{array}$

T. 22 N., R. 9 W.

$\begin{array}{rrrrr}0-500 & 21,004 & 21.1 & 783.72 & \\ 500-1,000 & 326 & 21.5 & 12.42 & 796.14\end{array}$

T. 22 N., R. $10 \mathrm{~W}$.

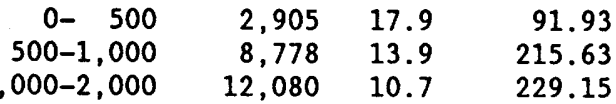

536.71

T. 22 N., R. $11 \mathrm{~W}$.

$\begin{array}{llll}500-1,000 & 3,525 & 10.6 & 66.32\end{array}$

$\begin{array}{llll}1,000-2,000 & 1,849 & 10.2 & 33.28\end{array}$

99.60
Table B2. Estimates of Federally owned subbituminous coal resources in the Menefee Formation, San Juan basin, New Mexico-Continued

\begin{tabular}{lccc}
\hline $\begin{array}{l}\text { Location and } \\
\text { overburden } \\
\text { thickness } \\
\text { (feet) }\end{array}$ & Acres & $\begin{array}{c}\text { Average } \\
\text { total } \\
\text { thick- } \\
\text { ness } \\
\text { (feet) }\end{array}$ & $\begin{array}{c}\text { Millions of } \\
\text { short tons }\end{array}$ \\
\hline & Santerval & Total \\
\hline
\end{tabular}

T. 23 N., R. 8 W. $0-500$

T. 23 N., R. 9 W. $\begin{array}{llll}0-500 & 20,492 & 12.0 & 435.29\end{array}$ $\begin{array}{lrrr}500-1,000 & 775 & 17.8 & 24.35\end{array}$

342.70

T. 23 N., R. 10 W. $\begin{array}{llll}0-500 & 2,706 & 11.0 & 52.60\end{array}$ $\begin{array}{llll}500-1,000 & 8,293 & 14.6 & 214.15\end{array}$ $\begin{array}{llll}1,000-2,000 & 15,053 & 13.3 & 353.91\end{array}$

620.66

T. $23 \mathrm{~N} .$, R. $11 \mathrm{~W}$. $\begin{array}{lllll}0-500 & 5,339 & 24.8 & 234.12\end{array}$ $\begin{array}{llll}500-1,000 & 5,519 & 20.9 & 204.42\end{array}$ $\begin{array}{llll}1,000-2,000 & 7,797 & 17.9 & 247.53\end{array}$

686.07

T. 23 N., R. 12 W.

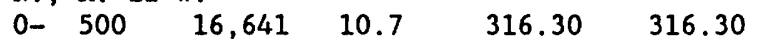

T. 23 N., R. 13 W.

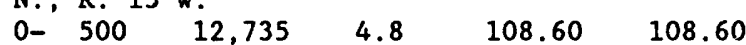

T. 24 N., R. 8 W.

$0-500$

737

9.9

12.84

12.84

T. 24 N., R. 9 W. O- 500

$500-1,000$

4,831

4,842

8.7

74.45

$1,000-2,000$

3,240

$2.2 \quad 12.72$

138.16

T. 24 N., R. 10 W.

$\begin{array}{rrrr}0-500 & 1,580 & 14.2 & 39.68 \\ 500-1,000 & 1,544 & 22.3 & 60.94 \\ 1,000-2,000 & 7,275 & 15.1 & 194.47 \\ 2,000-3,000 & 9,894 & 6.6 & 115.13\end{array}$

410.22

T. 24 N., R. 11 W.

$\begin{array}{lllll}0-500 & 5,818 & 28.1 & 289.71\end{array}$

500-1,000 $7,256 \quad 23.4 \quad 300.96$

$\begin{array}{rrrr}1,000-2,000 & 2,119 & 5.4 & 20.19\end{array}$

610.86

T. 24 N., R. 12 W.

$\begin{array}{rrrr}0-500 & 10,869 & 22.2 & 427.79 \\ 500-1,000 & 404 & 13.9 & 9.92\end{array}$

437.71

T. 24 N., R. 13 w.

$0-500$

838

5.5

8.11

8.11

T. $25 \mathrm{~N},$, R. $11 \mathrm{~W}$.

$\begin{array}{llll}1,000-2,000 & 6,053 & 11.8 & 126.59\end{array}$

$\begin{array}{llll}2,000-3,000 & 7,744 & 11.5 & 157.24\end{array}$

283.83

T. 25 N., R. 12 W.

$0-500$

$500-1,000$

789

13.1

18.34

$1,000-2,000$

2,450

18.8

17.8

58.96

76.98 
Table B2. Estimates of Federally owned subbituminous coal resources in the Menefee Formation, San Juan basin, New Mexico-Continued

\begin{tabular}{|c|c|c|c|c|}
\hline \multirow{2}{*}{$\begin{array}{l}\text { Location and } \\
\text { overburden } \\
\text { thickness } \\
\text { (feet) }\end{array}$} & \multirow{2}{*}{ Acres } & \multirow{2}{*}{$\begin{array}{c}\text { Average } \\
\text { total } \\
\text { thick- } \\
\text { ness } \\
\text { (feet) }\end{array}$} & \multicolumn{2}{|c|}{$\begin{array}{l}\text { Millions of } \\
\text { short tons }\end{array}$} \\
\hline & & & $\begin{array}{c}\text { Each } \\
\text { Interval }\end{array}$ & Total \\
\hline
\end{tabular}

San Juan County--Continued

\begin{tabular}{|c|c|c|c|c|}
\hline $\begin{array}{r}2,000-3,000 \\
>3,000\end{array}$ & $\begin{array}{l}1,417 \\
7,731\end{array}$ & $\begin{array}{l}7.6 \\
4.2\end{array}$ & $\begin{array}{l}18.93 \\
57.96\end{array}$ & 76.89 \\
\hline T. 27 N. , R. ${ }^{11}$ & W. 9,380 & 12.0 & 198.49 & 198.49 \\
\hline T. $\begin{array}{c}28 \text { N. } \\
>3,000\end{array}$ & 331 & 23.1 & 13.54 & 13.54 \\
\hline T. 28 N. , R. 11 & W. 15,333 & 11.6 & 315.34 & 315.34 \\
\hline $\begin{array}{c}\text { T. } 28 \text { N., R. } 12 \\
2,000-3,000 \\
>3,000\end{array}$ & $\begin{array}{r}2,519 \\
465\end{array}$ & $\begin{array}{l}7.9 \\
3.0\end{array}$ & $\begin{array}{r}35.06 \\
2.45\end{array}$ & 37.51 \\
\hline T. 29 N., R. ${ }^{11}$ & W. 2,040 & 8.1 & 29.11 & 29.11 \\
\hline T. $\begin{array}{c}30 \text { N. } \\
>3,000\end{array}$ & พ. 2,659 & 6.0 & 28.22 & 28.22 \\
\hline T. 31 N., R. ${ }^{11}$ & W. 15,976 & 6.4 & 181.27 & 181.27 \\
\hline T. $\begin{array}{c}32 \text { N. , R. } \\
>3,000\end{array}$ & W. 3,379 & 7.1 & 42.19 & 42.19 \\
\hline Total for San & & & & $7,664.84$ \\
\hline
\end{tabular}

Sandoval County

T. 16 N., R. 3 W. $0-\begin{array}{lllll}500 & 4,735 & 6.5 & 54.59 & 54.59\end{array}$

T. 16 N., R. 4 W.

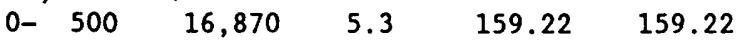

T. 19 N., R. 1 W. 500-1,000 $1,000-2,000$

$2,000-3,000$

$$
\begin{array}{r}
4,242 \\
440 \\
13,694
\end{array}
$$$$
\begin{aligned}
& 5.5 \\
& 6.5
\end{aligned}
$$$$
\begin{aligned}
& 6.5 \\
& 5.0
\end{aligned}
$$$$
41.57
$$$$
5.02
$$$$
120.55
$$

167.14

T. 19 N., R. 2 W.

$$
\begin{array}{r}
0-500 \\
500-1,000 \\
1,000-2,000
\end{array}
$$$$
2,925
$$$$
5,088
$$$$
6.5
$$$$
33.67
$$$$
3,091
$$

T. 19 N., R. 3 W.

$$
0-500
$$

500-1,000

$$
\begin{array}{r}
2,108 \\
7,212 \\
13,248
\end{array}
$$

$1,000-2,000$

254.53

T. 19 N., R. 4 W. $500-1,000$
$1,000-2,000$
$2,000-3,000$
2,693

4,969 1,184
8.7

7.8

5.3
41.21

68.79

11.09
Table B2. Estimates of Federally owned subbituminous coal resources in the Menefee Formation, San Juan basin, New Mexico-Continued

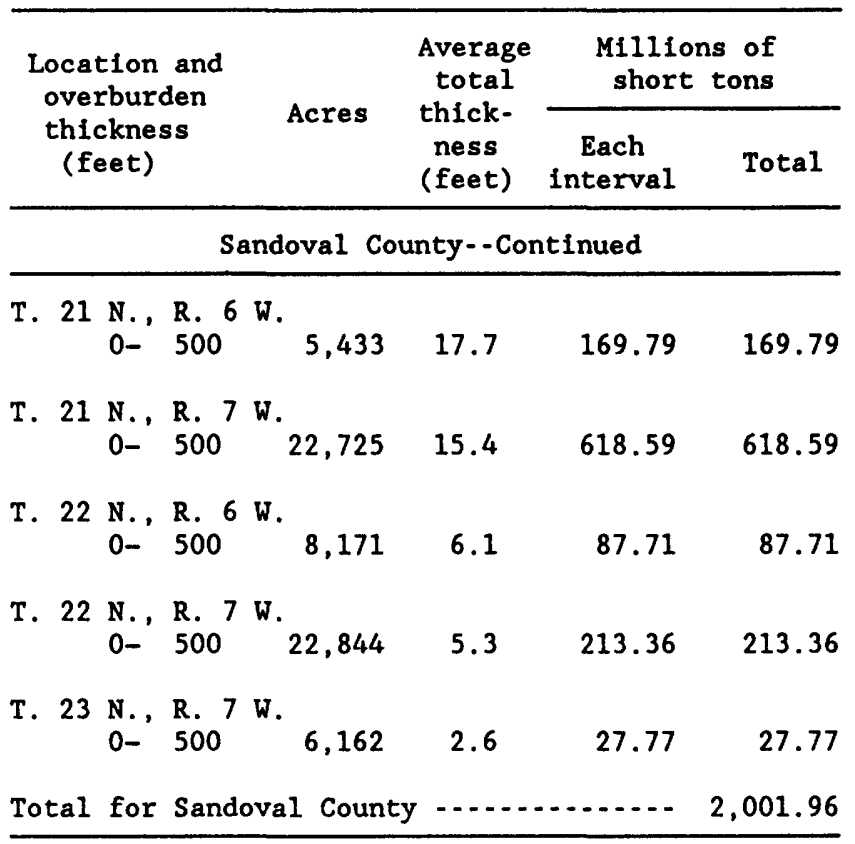

\section{REFERENCES CITED}

Crowley, S.S., Medlin, A.L., and Smith, R.J., 1981, Guide for the calculation of coal resources using the computer program GARNET: U.S. Geological Survey Open-File Report $81-563,34 \mathrm{p}$.

Fassett, J.E., and Hinds, J.S., 1971, Geology and fuel resources of the Fruitland Formation and Kirtland Shale of the San Juan basin, New Mexico and Colorado: U.S. Geological Survey Professional Paper 676, 76 p.

Krohn, K.K., Carey, M.A., Carter, M.D., and Medlin, A.L., 1981, Guide to the use of PACER, the data retrieval and update system for the National Coal Resources Data System (NCRDS): U.S. Geological Survey Open-File Report 82-291, 99 p.

Wood, G.H., Kehn, T.M., Carter, M.D., and Culbertson, W.C., 1983, Coal resource classification system of the U.S. Geological Survey: U.S. Geological Survey Circular 891, $65 \mathrm{p}$. 
Chapter C

\section{Coal Resource Estimation in the San Juan Basin Using Geostatistical Methods}

By LEWIS W. BOGER, JR., and ANTOINETTE L. MEDLIN

Resources of the Fruitland coal zone

estimated by the kriging technique

U.S. GEOLOGICAL SURVEY BULLETIN 1972

COALFIELDS OF NEW MEXICO: GEOLOGY AND RESOURCES 


\title{
CONTENTS
}

\author{
Abstract $\quad 37$ \\ Introduction 37 \\ Geostatistical methods 37 \\ Data preparation $\quad 37$ \\ Model selection $\mathbf{3 8}$ \\ Results 39 \\ Conclusions $\quad 42$ \\ References cited \\ 43
}

\section{FIGURES}

C1. Semi-variogram for coal thickness in the Fruitland Formation 38

C2-C5. Maps of the area underlain by the Fruitland Formation in the San Juan basin showing:

C2. Locations of data collection points and grid blocks used for kriging $\quad 39$

C3. Isopachs of kriged coal thickness values $\mathbf{4 0}$

C4. Estimate of thickness variance of coal beds, based on original kriged data 42

C5. Modified estimate of thickness variance of coal beds, generated by adding data for theoretical drill holes

43

\section{TABLES}

C1. Kriged coal tonnages and kriging variance for $10 \times 10-\mathrm{km}$ blocks in the area underlain by the Fruitland Formation, San Juan basin, New Mexico 


\title{
Coal Resource Estimation in the San Juan Basin Using Geostatistical Methods
}

\author{
By Lewis W. Boger, Jr., and Antoinette L. Medlin,
}

\begin{abstract}
This chapter describes the application of the geostatistical method known as kriging to estimate coal resources of the Fruitland coal zone in the San Juan basin. The study should be considered experimental because the method was used to estimate resources over a very large area (7,500 square miles), and the data were not homogeneous.

A semi-variogram model for coal thickness was developed for the entire Fruitland Formation (Upper Cretaceous). It was used to estimate the coal resources in the deposit using large $10 \times 10-\mathrm{km}(6.2 \times 6.2-\mathrm{mi})$ panels. A brief test also shows how maps of kriging variance can be used to project how much the resource estimate could be improved by additional data-collection points in particular locations. In addition, we attempted to calculate semi-variograms for ash, sulfur, and Btu, but the data were insufficient to support this effort.

Total in-place coal resources of the Fruitland Formation estimated by this technique were 159,984 million tons, which compares favorably with the GARNET isopach technique result of 167,501 million tons derived by Biewick and others (this volume, chap. B). However, since this study is experimental, further work is necessary before these results should be used for purposes other than observing trends, designing sampling programs, or gross estimates of in-place coal.
\end{abstract}

\section{INTRODUCTION}

The geostatistical method known as kriging is a useful technique for estimating coal tonnages at a point or over an area of ground. Kriging makes use of a semivariogram model to create weighting functions for values (in this case, coal thicknesses) at observed sample points.

Manuscript approved for publication January 10, 1991.
Estimated thickness may be calculated by summing the products of the sample values and their respective weights. Weighting functions are determined so that the estimate is unbiased and has minimum error variance. Variance is a measure of the difference between the value expected and the observed value. A good overview of the technique can be found in the first four chapters of Mousset-Jones (1980); for a detailed explanation see Journel and Huijbregts (1978).

A geostatistical software system on the NCRDS Prime 9750 computer was used to create a semi-variogram model and perform kriging for coal thickness data, to attempt to define semi-variograms for ash, sulfur, and Btu, and to create kriging variance maps. The following sections present further details of data preparation, semi-variogram model selection, results, and conclusions.

\section{GEOSTATISTICAL METHODS}

\section{Data Preparation}

A geostatistical analysis leading to the estimation of the quantity of a coal resource requires geologic analysis of the region and separation of the data into geologically homogeneous groups. When the data cannot be so grouped, the quality of the estimates will be lower. Standard statistical studies of the data are done to assure a reasonable degree of statistical homogeneity within subsets.

The same PACER data set retrieved from NCRDS for the GARNET estimation (Biewick and others, this volume) was used in the geostatistical estimation. Only drill hole data representing the complete Fruitland coal zone were used. Outcrop data and measured sections were eliminated to improve the homogeneity in the data set. The data were converted from the PACER format to a format suitable for input to the geostatistical programs. In this conversion, latitudes and longitudes were converted to kilometers in the Universal Transverse Mercator Coordinate System. The 
data set was then subjected to standard statistical tests mentioned above to ascertain the consistency of the data, and it was checked for any points with identical locations. Duplicate locations will cause the kriging program to fail. Coal thicknesses were summed for all samples with the same identifier (drill-hole name), yielding total Fruitland coal thicknesses identical to those that were used in the GARNET estimate.

\section{Model Selection}

Because an underlying premise of geostatistics is that the variable of interest has some degree of continuity within the area of study, a semi-variogram model must be selected which best depicts this continuity. The semi-variogram is a curve representing the degree of continuity of the variable: in this case, coal thickness (fig. C1). For this application, gamma $H$, the value of the semi-variogram, is defined as one-half the mean squared difference in total coal thickness (in feet) between drill holes located a given distance apart. In general, the semi-variogram is an increasing function of distance because, usually, the farther samples are from one another, the greater the difference between the sample values.

The semi-variogram of thickness shown in figure $\mathrm{C} 1$ was constructed from the Fruitland data set. In this case, at a distance of separation of $6.5 \mathrm{mi}$, determined by the flattening of this curve, the values become independent of one another. This plateau of constant variance is termed the "sill," and the horizontal distance at which the sill is reached is called the "range." The range defines the area of spatial influence within a given data set. The $y$-intercept is termed the "nugget." The nugget is a component of uncertainty that is not statistically predictable from the available data. The nugget comprises variability on a scale smaller than can be quantified given the spacing of the data samples, errors in the interpretation of the geophysical logs, and other factors characterized as noise.

The data available from the NCRDS detailed geochemical data base (USCHEM) for the Upper Cretaceous Fruitland Formation were not sufficient in number or distribution to develop any sort of statistical model for any of the basic coal quality parameters. Additional data were retrieved from a second coal quality data base (USALYT), which contains only data from standard proximate and

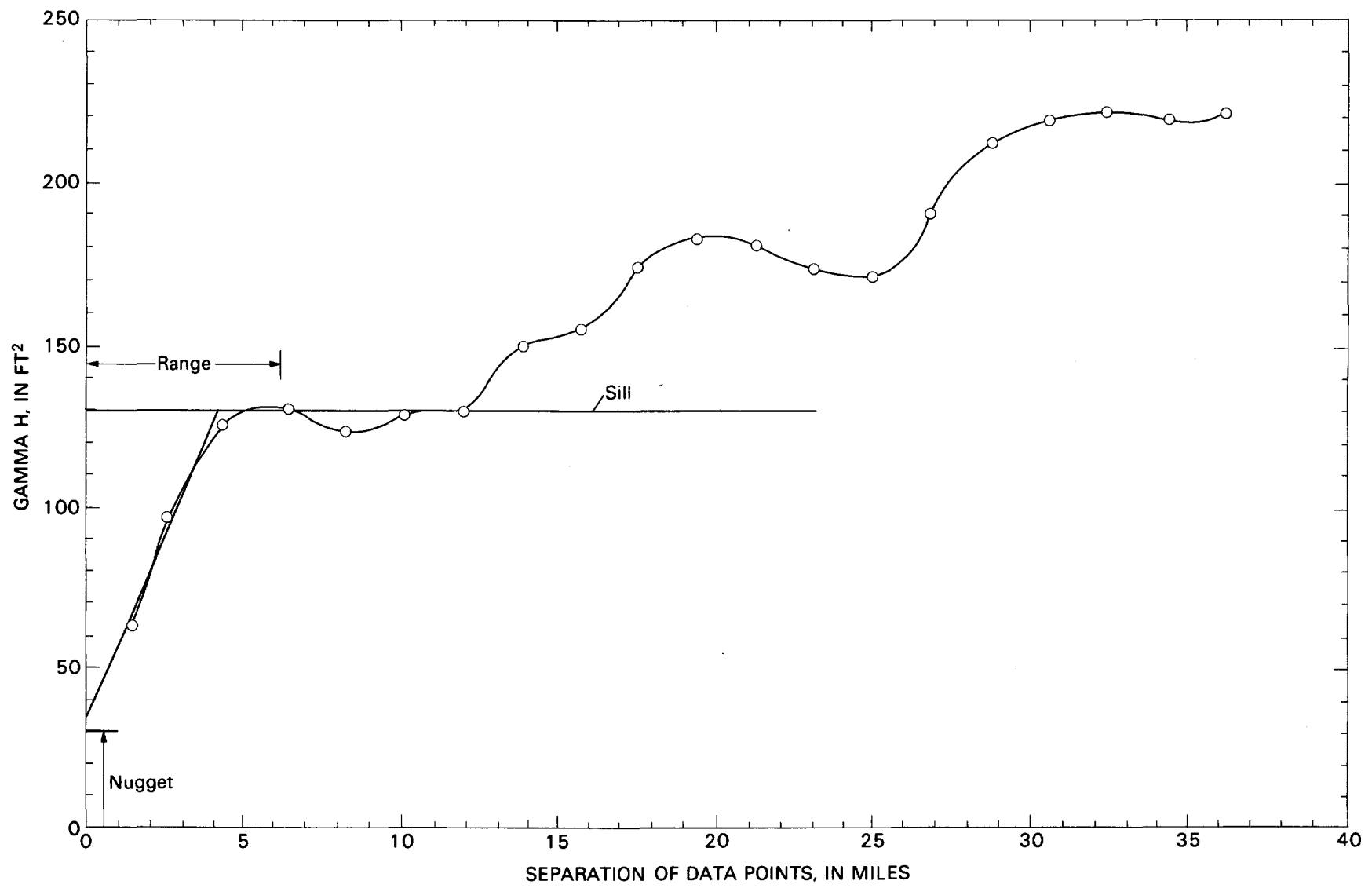

Figure C1. Semi-variogram for coal thickness in the Fruitland Formation, San Juan basin, New Mexico. See text for explanation of "gamma H," "nugget," "range," and "sill." 
ultimate coal analyses. This data set included data from 107 Fruitland Formation coal samples, but they were all clustered in the vicinity of the Navajo mine in the northwest corner of the basin. Again the distribution was insufficient to support the kriging analysis for the Fruitland coal. Several models for the distribution of Btu, ash, and sulfur values were tested, but the "best" model had a range of less than $2 \mathrm{mi}$. This result indicates that the data are essentially only valid for the point locations they represent; no reliable basin models could be developed.

\section{Results}

The kriging technique was developed for use in mine planning and development. It produces the most reliable estimates when applied to relatively small, geologically homogeneous areas where the distribution of data is fairly uniform.

Using the kriging technique to determine the coal resources of a large area where the coal beds are thin and irregular is an experimental application of the method.

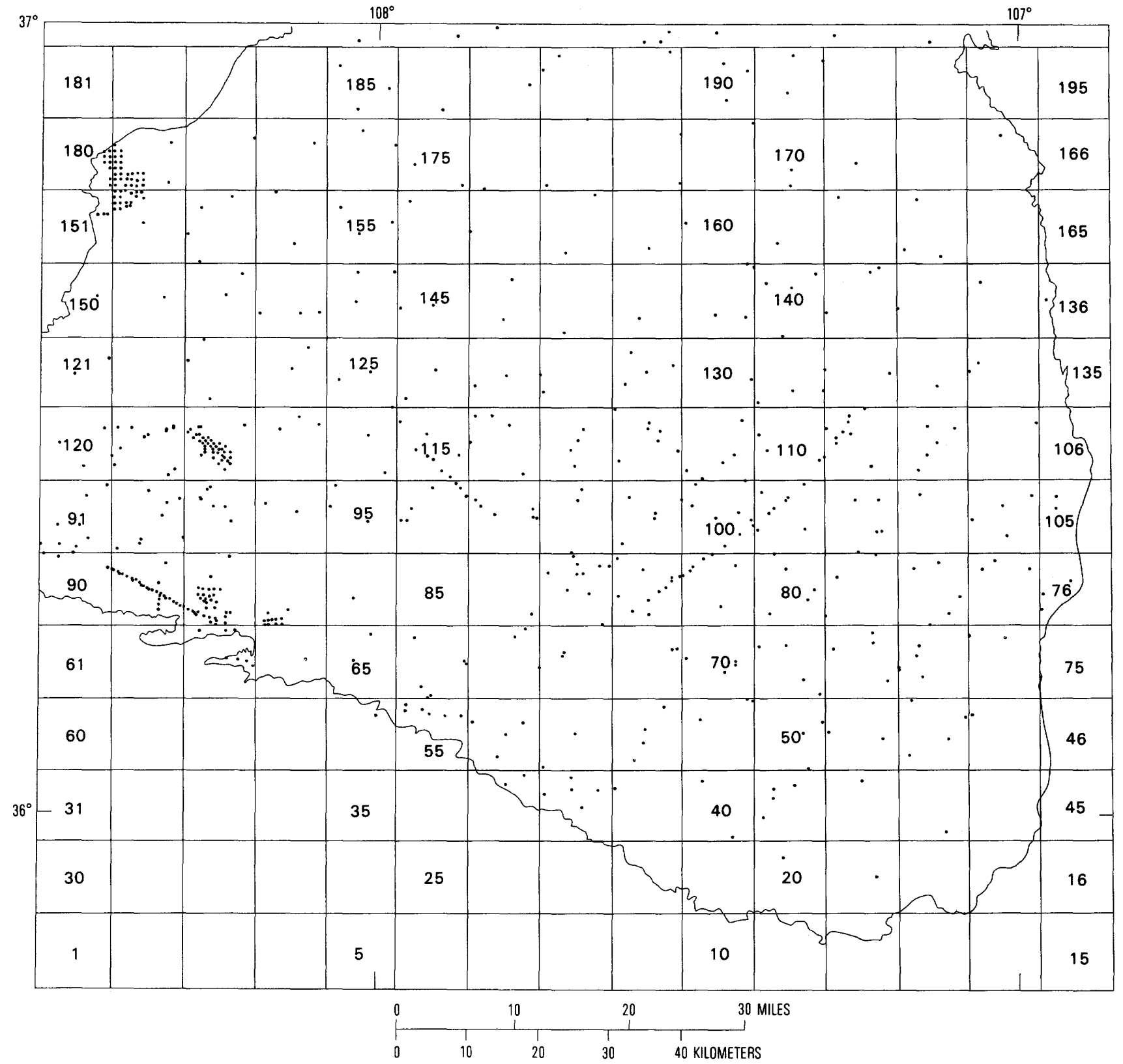

Figure C2. Locations of data collection points and $10 \times 10-\mathrm{km}(6.2 \times 6.2-\mathrm{mi})$ blocks used for kriging to estimate coal resources in the Fruitland Formation, San Juan basin, New Mexico (table C1). See figure B1 of Biewick and others (this volume, chap. B) for location of area shown. 


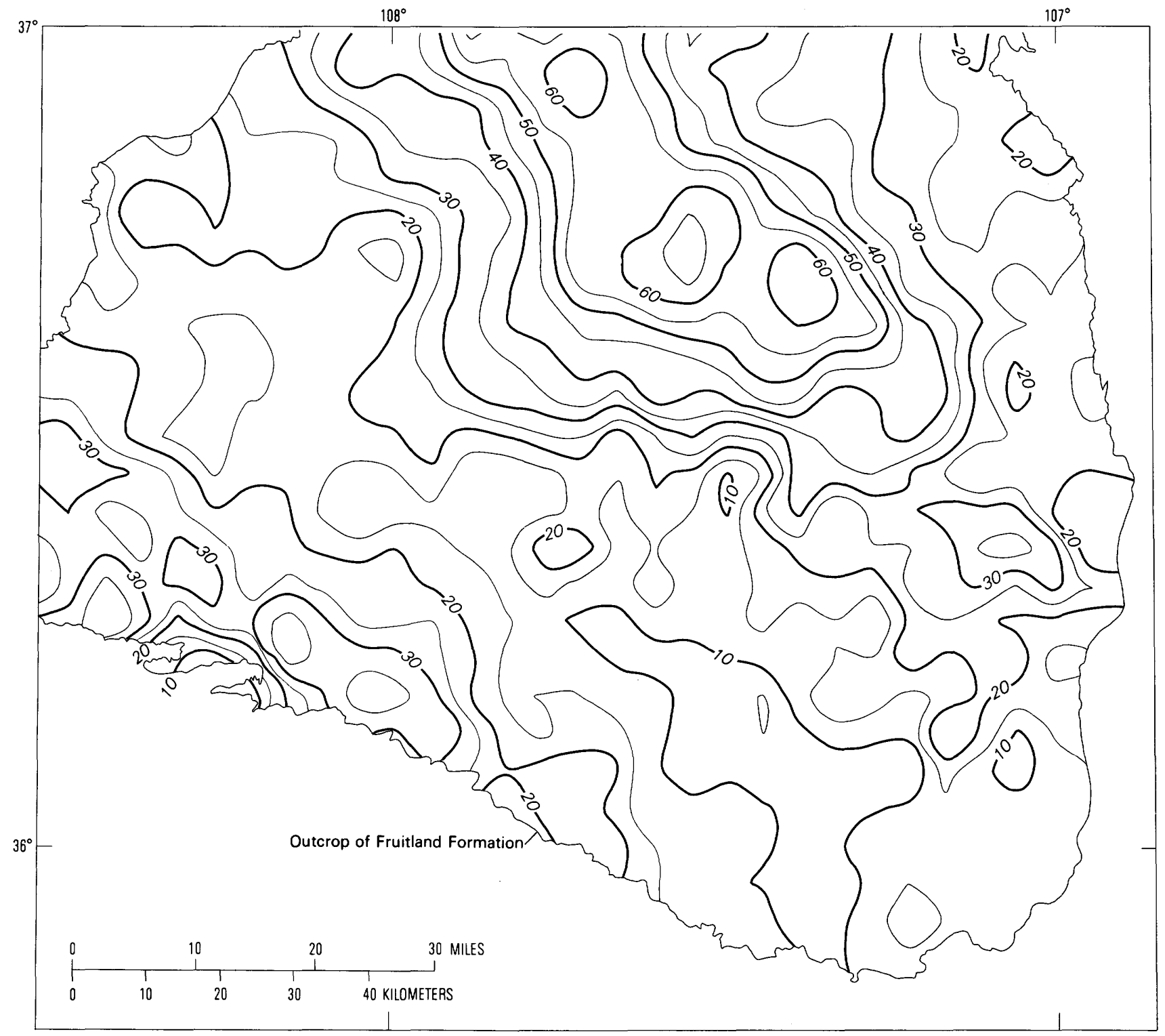

Figure C3. Isopachs of kriged coal thickness values, in feet, for the Fruitland coal zone in the San Juan basin, New Mexico. See figure B1 of Biewick and others (this volume, chap. B) for location of area shown.

Further work is necessary before the results should be used for purposes other than observing trends, designing sampling programs, or gross estimates of in-place coal.

The range, sill, and nugget parameters obtained from the construction of the semi-variogram were used in the kriging to estimate coal thickness at the grid intersections of a regularly spaced grid (fig. C2). The coal isopach map derived from these points (fig. C3) is generally similar to the one generated by the GARNET program (Biewick and others, this volume, fig. B2); the differences are due to the different gridding algorithms and interpolations used in the two techniques. The kriging algorithm also produces variance estimates at the grid nodes, which were contoured to produce the variance map in figure $\mathrm{C} 4$. This map represents the general reliability of the isopach map by indicating the potential variation of the estimated values. High variance values can be seen on the southern and western edges, in the northeast corner, and in the southcentral parts of the contoured area. This high variance probably reflects the lack of data in these areas.

Because kriging variance is not a function of the values encountered at sample locations but a function of the number of sample points, their relative locations, and the spatial variability model, maps of the variance can be very useful in designing future data collection efforts. Hypothetical drill-hole locations can be inserted to assess their impact on the precision of the estimate. Eight such hypothetical drill-hole locations were added to the data set 
Table C1. Kriged coal tonnages and kriging variance for $10 \times 10-\mathrm{km}(6.2 \times 6.2-\mathrm{mi})$ blocks of the Fruitland Formation, San Juan basin, New Mexico

\begin{tabular}{|c|c|c|c|c|c|c|c|c|c|c|c|}
\hline $\begin{array}{l}\text { B1ock } \\
\text { No. } \\
\text { (fig. } \\
\text { C2) }\end{array}$ & $\begin{array}{c}\text { Kriged } \\
\text { thick- } \\
\text { ness } \\
\text { (feet) }\end{array}$ & $\begin{array}{l}\text { Coal } \\
\text { (millions } \\
\text { of short } \\
\text { tons) }\end{array}$ & $\begin{array}{l}\text { Kriging } \\
\text { vari- } \\
\text { ance } \\
\left(f t^{2}\right)\end{array}$ & $\begin{array}{l}\text { Block } \\
\text { No. } \\
\text { (fig. } \\
\text { C2) }\end{array}$ & $\begin{array}{c}\text { Kriged } \\
\text { thick- } \\
\text { ness } \\
\text { (feet) }\end{array}$ & $\begin{array}{l}\text { Coal } \\
\text { (millions } \\
\text { of short } \\
\text { tons) }\end{array}$ & $\begin{array}{l}\text { Kriging } \\
\text { vari- } \\
\text { ance } \\
\left(f t^{2}\right)\end{array}$ & $\begin{array}{c}\text { Block } \\
\text { No. } \\
\text { (fig. } \\
\text { C2) }\end{array}$ & $\begin{array}{c}\text { Kriged } \\
\text { thick- } \\
\text { ness } \\
\text { (feet) }\end{array}$ & $\begin{array}{l}\text { Coal } \\
\text { (millions } \\
\text { of short } \\
\text { tons) }\end{array}$ & $\begin{array}{l}\text { Kriging } \\
\text { vari- } \\
\text { ance } \\
\left(f t^{2}\right)\end{array}$ \\
\hline $\begin{array}{l}20 \\
37 \\
38 \\
39 \\
40\end{array}$ & $\begin{array}{r}6.1 \\
19.5 \\
21.9 \\
14.1 \\
12.3\end{array}$ & $\begin{array}{l}268.20 \\
854.45 \\
957.02 \\
616.35 \\
538.28\end{array}$ & $\begin{array}{l}53 \\
58 \\
21 \\
39 \\
41\end{array}$ & $\begin{array}{l}93 \\
94 \\
95 \\
96 \\
97\end{array}$ & $\begin{array}{l}27.4 \\
20.6 \\
16.0 \\
15.9 \\
17.8\end{array}$ & $\begin{array}{r}1,196.52 \\
898.89 \\
699.58 \\
693.41 \\
776.47\end{array}$ & $\begin{array}{l}7 \\
21 \\
19 \\
18 \\
16\end{array}$ & $\begin{array}{l}144 \\
145 \\
146 \\
147 \\
148\end{array}$ & $\begin{array}{l}40.0 \\
26.3 \\
18.0 \\
16.8 \\
15.0\end{array}$ & $\begin{array}{r}1,751.51 \\
1,749.20 \\
786.00 \\
733.87 \\
655.93\end{array}$ & $\begin{array}{l}24 \\
14 \\
13 \\
13 \\
15\end{array}$ \\
\hline $\begin{array}{l}41 \\
42 \\
43 \\
47 \\
48\end{array}$ & $\begin{array}{r}8.7 \\
6.2 \\
20.4 \\
8.5 \\
14.7\end{array}$ & $\begin{array}{l}381.08 \\
269.99 \\
892.72 \\
373.34 \\
644.43\end{array}$ & $\begin{array}{l}10 \\
58 \\
83 \\
32 \\
13\end{array}$ & $\begin{array}{r}98 \\
99 \\
100 \\
101 \\
102\end{array}$ & $\begin{array}{l}18.1 \\
15.1 \\
13.6 \\
22.1 \\
24.0\end{array}$ & $\begin{array}{r}789.98 \\
658.34 \\
593.43 \\
966.38 \\
1,049.92\end{array}$ & $\begin{array}{r}10 \\
10 \\
8 \\
4 \\
14\end{array}$ & $\begin{array}{l}149 \\
150 \\
151 \\
152 \\
153\end{array}$ & $\begin{array}{l}16.6 \\
18.4 \\
11.9 \\
19.9 \\
19.5\end{array}$ & $\begin{array}{l}727.70 \\
805.56 \\
519.73 \\
868.32 \\
853.49\end{array}$ & $\begin{array}{l}27 \\
50 \\
52 \\
24 \\
12\end{array}$ \\
\hline $\begin{array}{l}49 \\
50 \\
51 \\
52 \\
53\end{array}$ & $\begin{array}{r}6.6 \\
7.7 \\
8.6 \\
12.0 \\
22.0\end{array}$ & $\begin{array}{l}289.02 \\
335.73 \\
374.92 \\
525.37 \\
964.32\end{array}$ & $\begin{array}{l}15 \\
27 \\
19 \\
13 \\
10\end{array}$ & $\begin{array}{l}103 \\
104 \\
105 \\
106 \\
107\end{array}$ & $\begin{array}{l}30.1 \\
34.0 \\
18.6 \\
12.3 \\
24.3\end{array}$ & $\begin{array}{r}1,314.83 \\
1,486.20 \\
811.77 \\
538.71 \\
1,062.51\end{array}$ & $\begin{array}{l}14 \\
11 \\
19 \\
71 \\
33\end{array}$ & $\begin{array}{l}154 \\
155 \\
156 \\
157 \\
158\end{array}$ & $\begin{array}{l}21.2 \\
16.1 \\
21.8 \\
36.6 \\
59.5\end{array}$ & $\begin{array}{r}928.94 \\
702.69 \\
952.25 \\
1,601.66 \\
2,600.19\end{array}$ & $\begin{array}{l}20 \\
13 \\
21 \\
27 \\
24\end{array}$ \\
\hline $\begin{array}{l}54 \\
55 \\
56 \\
62 \\
63\end{array}$ & $\begin{array}{r}20.6 \\
28.2 \\
29.2 \\
11.7 \\
9.4\end{array}$ & $\begin{array}{r}902.52 \\
1,232.48 \\
1,274.99 \\
510.11 \\
410.78\end{array}$ & $\begin{array}{r}10 \\
31 \\
83 \\
120 \\
31\end{array}$ & $\begin{array}{l}108 \\
109 \\
110 \\
111 \\
112\end{array}$ & $\begin{array}{l}28.0 \\
33.2 \\
29.8 \\
14.6 \\
20.1\end{array}$ & $\begin{array}{r}1,223.55 \\
1,452.61 \\
1,302.98 \\
638.35 \\
877.85\end{array}$ & $\begin{array}{r}11 \\
6 \\
7 \\
8 \\
10\end{array}$ & $\begin{array}{l}159 \\
160 \\
161 \\
162 \\
163\end{array}$ & $\begin{array}{l}67.4 \\
67.1 \\
59.5 \\
42.7 \\
25.1\end{array}$ & $\begin{array}{l}2,945.76 \\
2,934.17 \\
2,600.84 \\
1,866.01 \\
1,096.10\end{array}$ & $\begin{array}{l}31 \\
28 \\
18 \\
26 \\
15\end{array}$ \\
\hline $\begin{array}{l}64 \\
65 \\
66 \\
67 \\
68\end{array}$ & $\begin{array}{l}21.5 \\
34.8 \\
30.5 \\
15.8 \\
14.1\end{array}$ & $\begin{array}{r}939.65 \\
1,521.62 \\
1.332 .20 \\
689.26 \\
618.71\end{array}$ & $\begin{array}{l}19 \\
18 \\
12 \\
17 \\
13\end{array}$ & $\begin{array}{l}113 \\
114 \\
115 \\
116 \\
117\end{array}$ & $\begin{array}{l}17.0 \\
12.7 \\
16.2 \\
14.0 \\
18.1\end{array}$ & $\begin{array}{l}744.15 \\
556.12 \\
706.93 \\
612.19 \\
792.74\end{array}$ & $\begin{array}{r}11 \\
10 \\
5 \\
16 \\
20\end{array}$ & $\begin{array}{l}164 \\
167 \\
168 \\
169 \\
170\end{array}$ & $\begin{array}{l}20.6 \\
11.3 \\
20.1 \\
30.5 \\
36.6\end{array}$ & $\begin{array}{r}902.56 \\
493.40 \\
878.33 \\
1,335.61 \\
1,600.35\end{array}$ & $\begin{array}{l}44 \\
68 \\
52 \\
22 \\
34\end{array}$ \\
\hline $\begin{array}{l}69 \\
70 \\
71 \\
72 \\
73\end{array}$ & $\begin{array}{r}9.2 \\
7.2 \\
12.2 \\
16.5 \\
18.6\end{array}$ & $\begin{array}{l}402.69 \\
317.05 \\
533.33 \\
720.18 \\
814.48\end{array}$ & $\begin{array}{r}36 \\
11 \\
9 \\
13 \\
5\end{array}$ & $\begin{array}{l}118 \\
119 \\
120 \\
121 \\
122\end{array}$ & $\begin{array}{l}15.5 \\
25.5 \\
32.7 \\
23.3 \\
19.7\end{array}$ & $\begin{array}{r}677.19 \\
1,113.64 \\
1,428.33 \\
1,018.91 \\
861.85\end{array}$ & $\begin{array}{r}14 \\
5 \\
15 \\
19 \\
27\end{array}$ & $\begin{array}{l}171 \\
172 \\
173 \\
174 \\
175\end{array}$ & $\begin{array}{l}47.5 \\
58.0 \\
58.6 \\
33.8 \\
31.7\end{array}$ & $\begin{array}{l}2,078.53 \\
2,534.71 \\
2,561.00 \\
1,477.23 \\
1,387.44\end{array}$ & $\begin{array}{l}19 \\
42 \\
31 \\
20 \\
12\end{array}$ \\
\hline $\begin{array}{l}74 \\
75 \\
76 \\
77 \\
78\end{array}$ & $\begin{array}{l}17.8 \\
11.8 \\
19.2 \\
20.7 \\
24.0\end{array}$ & $\begin{array}{r}778.22 \\
515.75 \\
840.94 \\
907.33 \\
1,050.40\end{array}$ & $\begin{array}{l}25 \\
83 \\
22 \\
11 \\
18\end{array}$ & $\begin{array}{l}123 \\
124 \\
125 \\
126 \\
127\end{array}$ & $\begin{array}{l}14.5 \\
15.6 \\
19.4 \\
24.0 \\
30.9\end{array}$ & $\begin{array}{r}636.03 \\
680.60 \\
848.42 \\
1,051.71 \\
1,351.92\end{array}$ & $\begin{array}{l}15 \\
20 \\
12 \\
12 \\
10\end{array}$ & $\begin{array}{l}176 \\
177 \\
178 \\
179 \\
180\end{array}$ & $\begin{array}{l}25.4 \\
22.8 \\
19.4 \\
19.6 \\
13.6\end{array}$ & $\begin{array}{r}1,110.84 \\
997.43 \\
848.02 \\
858.08 \\
595.00\end{array}$ & $\begin{array}{r}24 \\
19 \\
25 \\
4 \\
48\end{array}$ \\
\hline $\begin{array}{l}79 \\
80 \\
81 \\
82 \\
83\end{array}$ & $\begin{array}{l}14.1 \\
13.8 \\
12.7 \\
11.9 \\
10.2\end{array}$ & $\begin{array}{l}617.70 \\
602.40 \\
556.78 \\
521.61 \\
445.38\end{array}$ & $\begin{array}{r}10 \\
12 \\
10 \\
5 \\
9\end{array}$ & $\begin{array}{l}128 \\
129 \\
130 \\
131 \\
132\end{array}$ & $\begin{array}{l}34.2 \\
34.2 \\
39.5 \\
44.0 \\
41.4\end{array}$ & $\begin{array}{l}1,494.29 \\
1,496.34 \\
1,728.98 \\
1,923.44 \\
1,811.12\end{array}$ & $\begin{array}{l}15 \\
10 \\
27 \\
10 \\
10\end{array}$ & $\begin{array}{l}181 \\
182 \\
183 \\
184 \\
\\
185\end{array}$ & $\begin{array}{l}17.4 \\
13.9 \\
23.5 \\
30.8 \\
36.6\end{array}$ & $\begin{array}{r}758.98 \\
608.65 \\
1,029.84 \\
1,345.54 \\
1,601.40\end{array}$ & $\begin{array}{r}116 \\
69 \\
37 \\
57\end{array}$ \\
\hline $\begin{array}{l}84 \\
85 \\
86 \\
87 \\
88\end{array}$ & $\begin{array}{l}12.2 \\
26.7 \\
29.3 \\
32.1 \\
24.4\end{array}$ & $\begin{array}{r}532.85 \\
1,167.88 \\
1,282.64 \\
1,404.28 \\
1,065.71\end{array}$ & $\begin{array}{r}52 \\
58 \\
23 \\
17 \\
7\end{array}$ & $\begin{array}{l}133 \\
134 \\
137 \\
138 \\
139\end{array}$ & $\begin{array}{l}36.9 \\
18.4 \\
16.8 \\
35.7 \\
51.9\end{array}$ & $\begin{array}{r}1,612.86 \\
803.89 \\
732.08 \\
1,559.28 \\
2,270.45\end{array}$ & $\begin{array}{l}16 \\
27 \\
45 \\
23 \\
17\end{array}$ & $\begin{array}{l}186 \\
187 \\
188 \\
\\
189 \\
190\end{array}$ & $\begin{array}{l}35.5 \\
54.8 \\
66.1\end{array}$ & $\begin{array}{l}1,001.40 \\
1,550.53 \\
2,396.98 \\
2,891.83\end{array}$ & $\begin{array}{l}22 \\
42 \\
25 \\
\\
48 \\
24\end{array}$ \\
\hline $\begin{array}{l}89 \\
90 \\
91 \\
92\end{array}$ & $\begin{array}{l}26.8 \\
30.7 \\
25.2 \\
25.4\end{array}$ & $\begin{array}{l}1,170.63 \\
1,340.73 \\
1,104.02 \\
1,112.02\end{array}$ & $\begin{array}{r}12 \\
45 \\
6 \\
8\end{array}$ & $\begin{array}{l}140 \\
141 \\
142 \\
143\end{array}$ & $\begin{array}{l}60.2 \\
53.2 \\
52.8 \\
48.5\end{array}$ & $\begin{array}{l}2,632.16 \\
2,325.26 \\
2,308.94 \\
2,119.78\end{array}$ & $\begin{array}{l}10 \\
27 \\
24 \\
25\end{array}$ & $\begin{array}{r}191 \\
192 \\
\text { Tot } \\
\end{array}$ & $\begin{array}{r}33.1 \\
30.2 \\
\quad \ldots 1\end{array}$ & $\begin{array}{l}1,449.15 \\
1,322.84 \\
59,983.71\end{array}$ & $\begin{array}{l}12 \\
46\end{array}$ \\
\hline
\end{tabular}

${ }^{1} \mathrm{Kriged}$ thlcknesses are rounded here, but were carried out to three decimal places for the calculation of coal tonnages. 


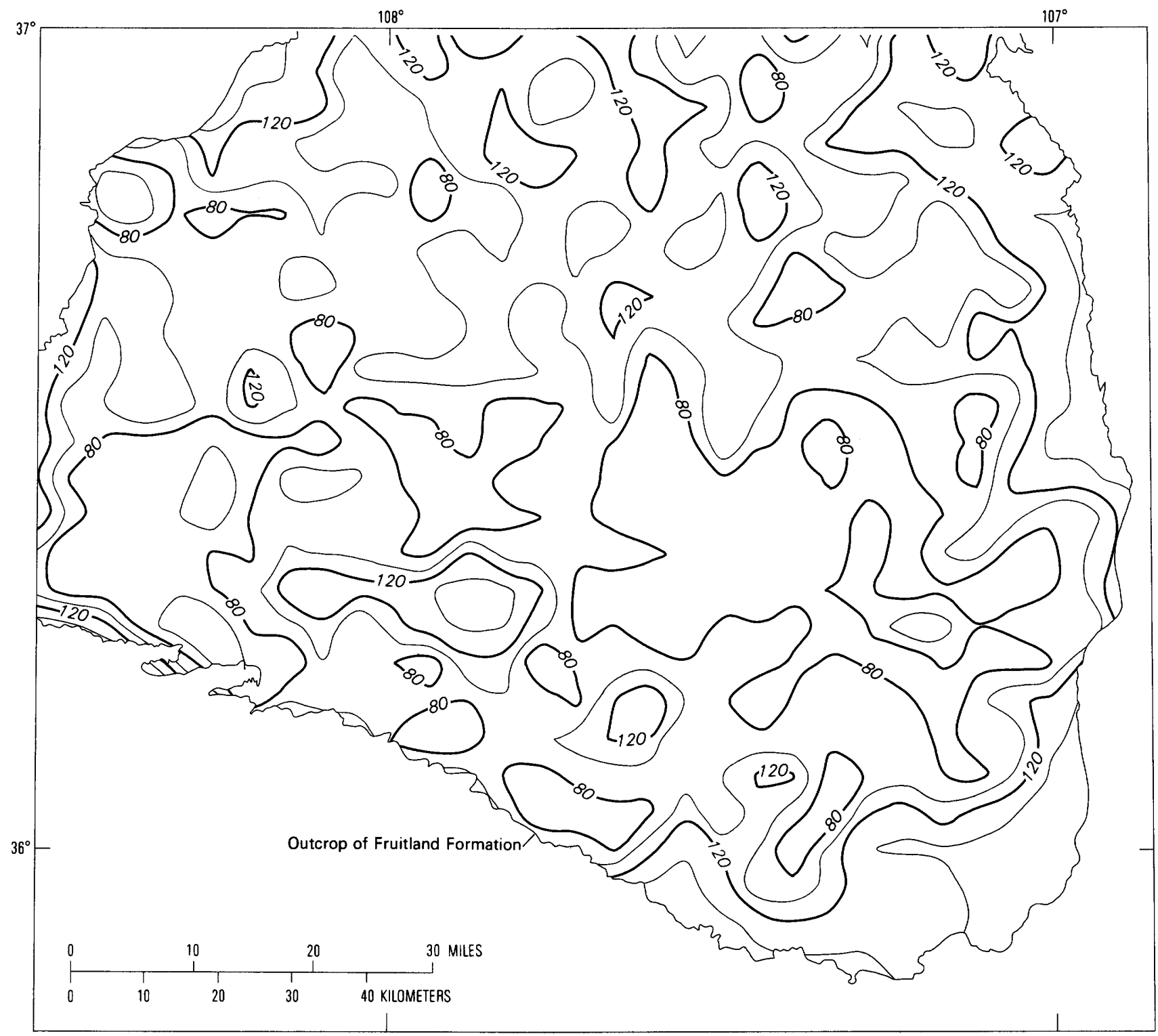

Figure C4. Estimate of thickness variance of coal beds in the Fruitland Formation, based on kriged original data. See figure B1 of Biewick and others (this volume, chap. B) for location of area shown.

in locations of high variance, and a second variance map was generated (fig. C5). A comparison of figures $\mathrm{C} 4$ and $\mathrm{C5}$ illustrates how much the estimate can be improved by a limited amount of selected drilling. (Note the southeastern, eastern, and northwest areas of the figures.)

Average coal thicknesses were computed for each $10 \times 10-\mathrm{km}(6.2 \times 6.2-\mathrm{mi})$ grid block within the basin (fig. C2) using block kriging. Coal tonnages were derived from these thicknesses using a value of $43,737,400$ tons per foot of thickness (rounded from $43,737,408$ tons/ft, which equals 1,770 tons/acre $\mathrm{ft} \times 247.104 \mathrm{acres} / \mathrm{km}^{2} \times 100 \mathrm{~km}^{2}$ per grid block). Results are shown in table C1. Blocks not listed there were not represented by enough data to allow kriging. Total in-place coal resources estimated by this method are
159,983 million tons; this compares favorably with the GARNET total coal estimate of 167,501 million tons-a difference of less than 5 percent.

\section{CONCLUSIONS}

As previously stated, the kriged results of this portion of the San Juan basin study should be considered with an understanding of their limitations:

1. The area of study is much larger than the typical mine site application. 


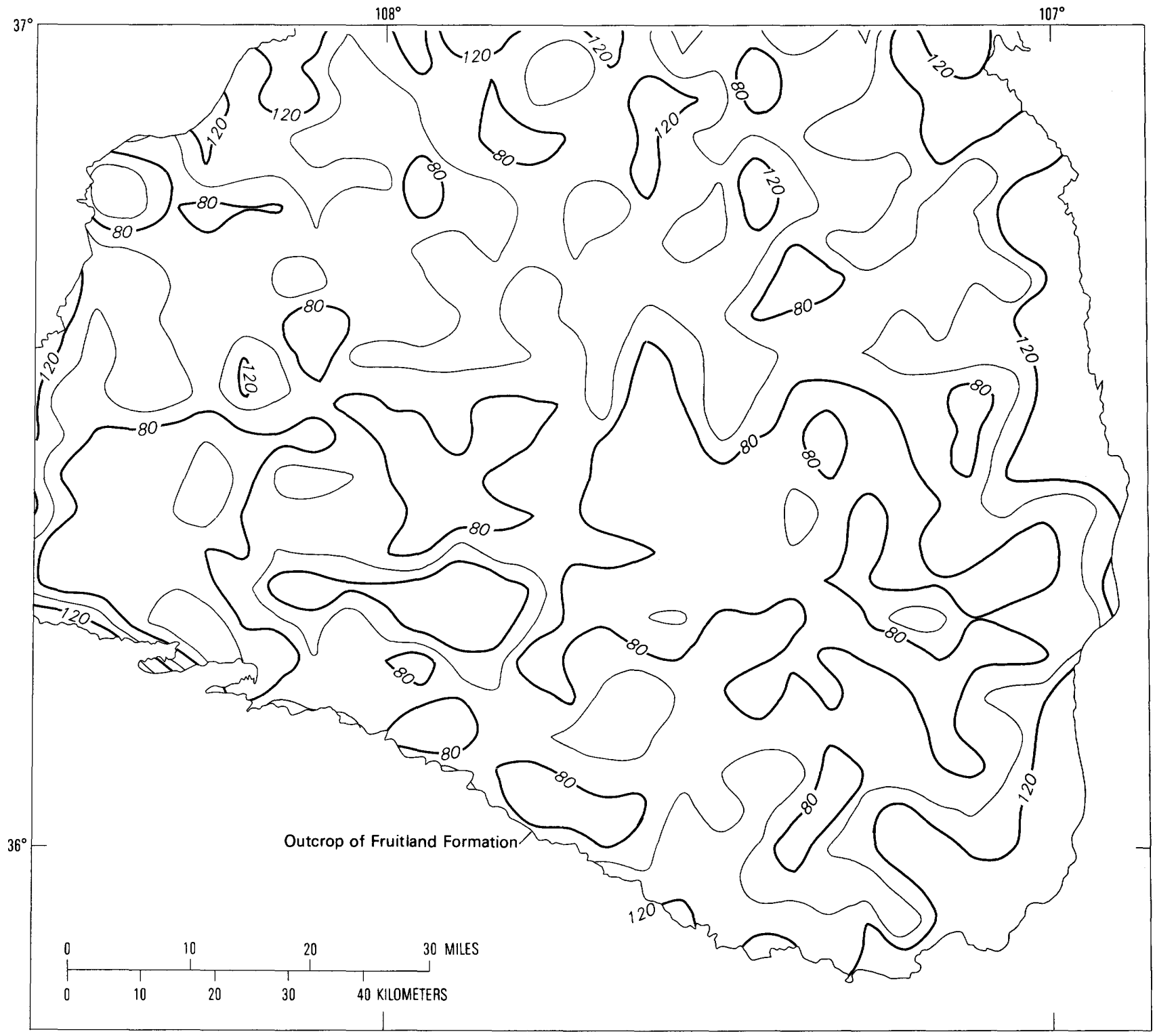

Figure C5. Modified estimate of thickness variance of coal beds in the Fruitland Formation, generated by adding data for eight theoretical drill holes to the original kriged data. Compare with figure C4.

2. The data set was constructed of summed thickness values and cannot be considered to be homogeneous.

3. The clustering of data in some areas and the scarcity of data in others bias the results.

4. Determining a single semi-variogram model for the entire deposit, rather than determining a separate semi-variogram model for each $10 \times 10$ $\mathrm{km}$ block, smooths possible localized variations within the area of this study.

5. Variances that are high relative to low tonnages shown for some blocks in table $\mathrm{Cl}$ are attributable to one or more of the above four items; they indicate unreliable estimates over some large areas of the basin.

A further demonstration of the kriging method would be better carried out in an area of similar size where more data are available and closer control can be imposed on data quality.

\section{REFERENCES CITED}

Journel, A.G., and Huijbregts, Ch.J., 1978, Mining geostatistics: New York, Academic Press, 600 p.

Mousset-Jones, P.F., ed., 1980, Geostatistics: New York, McGraw-Hill, 168 p. 
Chapter D

\section{Geology and Coal Resources of the Raton Coalfield}

\section{By CHARLES L. PILLMORE}

Stratigraphic and environmental

framework of an area of coal-bearing

Late Cretaceous and Paleocene rocks

U.S. GEOLOGICAL SURVEY BULLETIN 1972

COALFIELDS OF NEW MEXICO: GEOLOGY AND RESOURCES 


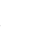




\title{
CONTENTS
}

\author{
Abstract 49 \\ Introduction 49 \\ Geologic setting $\mathbf{5 2}$ \\ Stratigraphy $\mathbf{5 3}$ \\ Pierre Shale and Trinidad Sandstone 53 \\ Vermejo Formation $\mathbf{5 4}$ \\ Raton Formation $\mathbf{5 5}$ \\ Poison Canyon Formation $\mathbf{5 5}$ \\ Igneous rocks $\mathbf{5 5}$ \\ Coal resources 56 \\ Coal deposits in the Vermejo Formation $\mathbf{5 8}$ \\ Castle Rock district $\mathbf{5 8}$ \\ Rosado district $\mathbf{5 9}$ \\ Brilliant district $\quad \mathbf{6 0}$ \\ Dawson and Koehler districts 61 \\ Other areas $\mathbf{6 1}$ \\ Coal deposits in the Raton Formation $\quad 61$ \\ York Canyon district $\mathbf{6 2}$ \\ Upper York (Left Fork) district 63 \\ Cottonwood Canyon district 63 \\ Ancho Canyon district 63 \\ Chimney Divide district 64 \\ Potato Canyon district $\mathbf{6 5}$ \\ Crow Canyon district $\mathbf{6 5}$ \\ Brilliant district $\mathbf{6 6}$ \\ Other coal beds in the Raton Formation $\mathbf{6 6}$
}

Conclusions $\quad 67$

References cited 67

\section{FIGURES}

D1. Location map of coal mines, abandoned coal mining towns and camps, and other geographic features in and near the Raton coalfield $\mathbf{5 0}$

D2. Map showing locations of the Raton and San Juan basins $\mathbf{5 2}$

D3. Stratigraphic chart of Upper Cretaceous and Paleocene rocks of the New Mexico portions of the Raton and San Juan basins $\mathbf{5 3}$

D4. Map showing areal distribution of coal-bearing Upper Cretaceous and Paleocene rocks in the Raton basin 53

D5. Generalized geologic map of the Raton coalfield $\mathbf{5 4}$

D6. Diagrammatic geologic cross section and columnar sections across the northwestern part of the Raton coalfield 56

D7. Map showing Western Interior seaway of Late Cretaceous time and locations of the Raton and San Juan basins $\mathbf{5 8}$

D8. Map of the Raton coalfield showing districts that have demonstrated coal resources 62

D9. Map of the Raton coalfield showing geographic subdivisions used to describe inferred coal resources 63

D10. Diagrammatic stratigraphic section showing relations of coal beds in the central and eastern parts of the Raton coalfield 


\section{TABLES}

D1. General stratigraphic description of the Raton coalfield $\mathbf{5 9}$

D2. Representative proximate analyses of selected coal beds in the Raton coalfield 60

D3. Ranges of volatile matter and sulfur in 164 core samples of the major coal beds in the Raton coalfield $\mathbf{6 0}$

D4. Demonstrated coal resources of commercial thickness within mining districts of the Raton coalfield 61

D5. Estimated minimum inferred coal resources within subdivisions of the Raton coalfield 


\title{
Geology and Coal Resources of the Raton Coalfield
}

\author{
By Charles L. Pillmore
}

\section{Abstract}

The Raton basin is a broad, arcuate depositional trough of structural origin that extends about 85 miles from near Gardner, Colo., to Cimarron, N. Mex. It is the southernmost of the Laramide intracratonic folded basins at the eastern margin of the Rocky Mountains and is a sister basin to the San Juan basin, which lies to the west and contains older but related rocks. The Raton coalfield is the New Mexico portion of the area within the Raton basin that is underlain by coal-bearing rocks. The coalfield covers an area of about 900 square miles and contains large resources of high-quality coking coal in rocks of the Vermejo Formation of Late Cretaceous age and the Raton Formation of Late Cretaceous and Paleocene age. Coal has been mined along the eastern margin of the coalfield since about 1870, but only the York Canyon surface mine and the Cimarron underground operation, in the north-central part of the coalfield, are presently active (1991). Coal beds of the Vermejo Formation were deposited in contemporaneous fluviodeltaic plain and back-barrier coastal environments and are the thickest and most extensive coal beds in the Raton field. The coal beds of the Raton Formation accumulated in poorly drained backswamp environments on flood plains of meandering streams that drained a broad alluvial plain. These coal deposits are lenticular in shape and less extensive than those of the Vermejo Formation.

Recent field and subsurface investigations within the coalfield permit delineation of 14 principal coal districts, based on the areal distribution of the thickest coal beds in both formations. Nearly 1.5 billion tons of demonstrated coal resources have been calculated for these districts. Castle Rock, the largest unmined district, is in the west-central part of the coalfield and contains about one-third of the demonstrated resources. The remainder of the resources are distributed among 13 smaller districts, located mostly in the northern part of the field. In addition to the demonstrated resources, about 8 billion tons of inferred resources are estimated for the Raton field-more than twice earlier figures. The inferred resources, which are mainly in thinner beds, are distributed throughout the coalfield in 15 geographic subdivisions.

Manuscript approved for publication January 10, 1991.

\section{INTRODUCTION}

The Raton coalfield occupies the New Mexico portion of the Raton Mesa coal region, the southernmost part of the Northern Great Plains coal province (Trumbull, 1959). The coalfield covers an area of about $900-1,000 \mathrm{mi}^{2}$ in northeastern New Mexico and contains large resources of high-quality coking coal in rocks of the Vermejo (Upper Cretaceous) and Raton (Upper Cretaceous and Paleocene) Formations. An early estimate (Read and others, 1950) tabulated 4.71 billion tons of coal in beds 14 in. thick or greater, beneath less than $3,000 \mathrm{ft}$ of overburden. A more recent appraisal indicates identified coal resources of about 9.5 billion tons, more than twice that earlier figure.

Coal was discovered in the coalfield by the Long Expedition in about 1821 (Lee, 1917) and has been mined commercially since about 1870 . Mining activity was concentrated along the eastern margins of the field until 1966, when the York Canyon mine was opened in the west-central part of the coalfield, about $26 \mathrm{mi}$ west of Raton. At present, the York Canyon surface mine and the Cimarron mine, about $6 \mathrm{mi}$ to the northwest, are the only active mines. The locations of these mines and other geographic features are shown on figure D1.

The following brief discussion of the location and general geologic setting of the Raton coalfield is from various reports by Pillmore (1969a; 1969b; 1976), Pillmore and others (1984), Pillmore and Flores $(1984 ; 1987)$, Wanek (1963), and Lee (1917). Resource estimates shown here have been augmented and changed from those calculated by Pillmore (1969b), using data supplied by Kaiser Coal Corp., for the large part of the coalfield in the coal districts and subdivisions west of Raton, and by Santa Fe Mining Corp. for the Yankee subdivision, east of Raton. I am indebted to both of these corporations for allowing use of their proprietary subsurface data to improve these estimates. Pittsburg \& Midway (P\&M) Coal Mining Co. purchased Kaiser's mines and coal resources in 1989. Updates on the resources and expected production of the York Canyon and Cimarron mines and the approval to publish this report are much appreciated. 


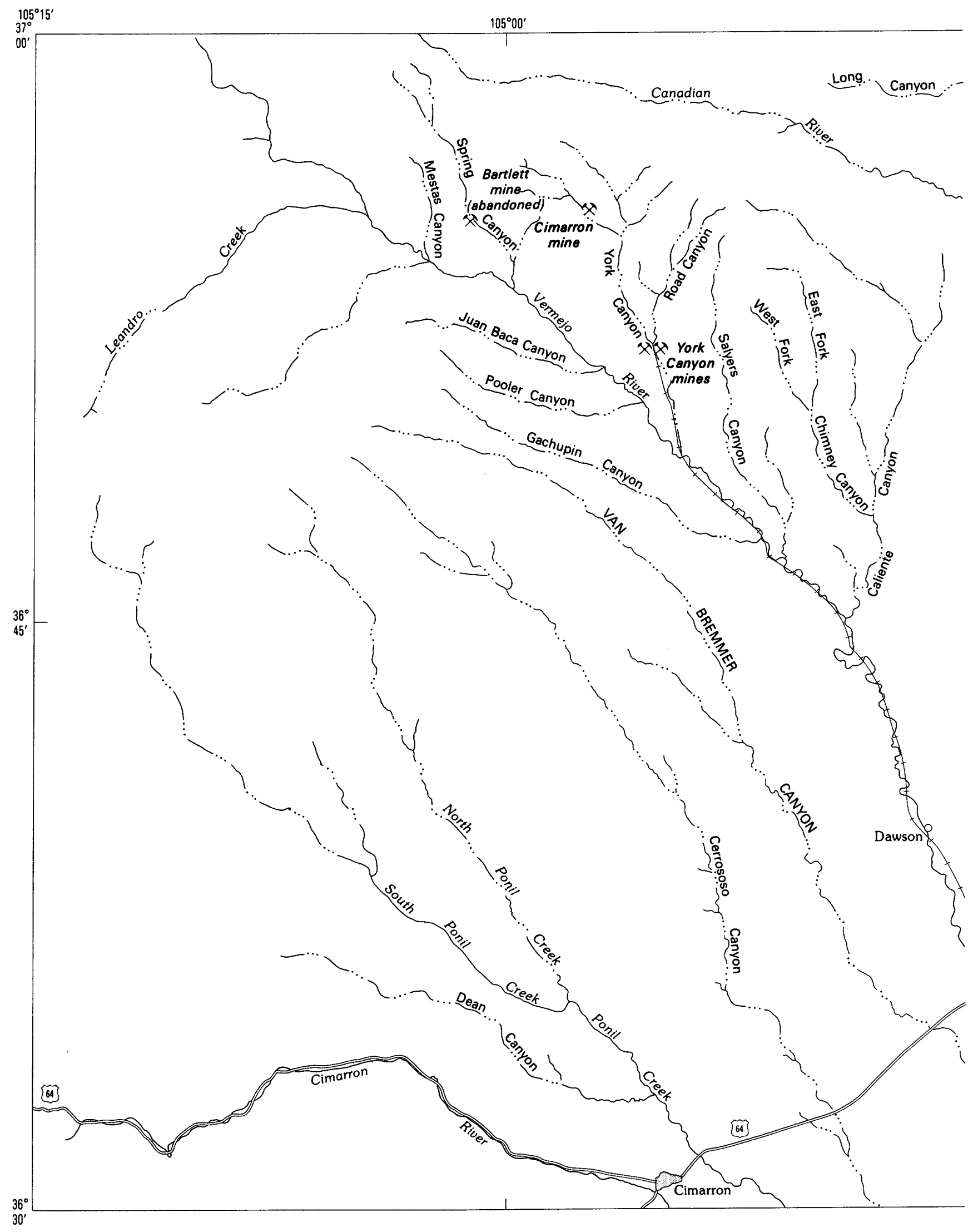

Figure D1. Location of coal mines, abandoned coal mining towns and camps, and other geographic features in and near the 


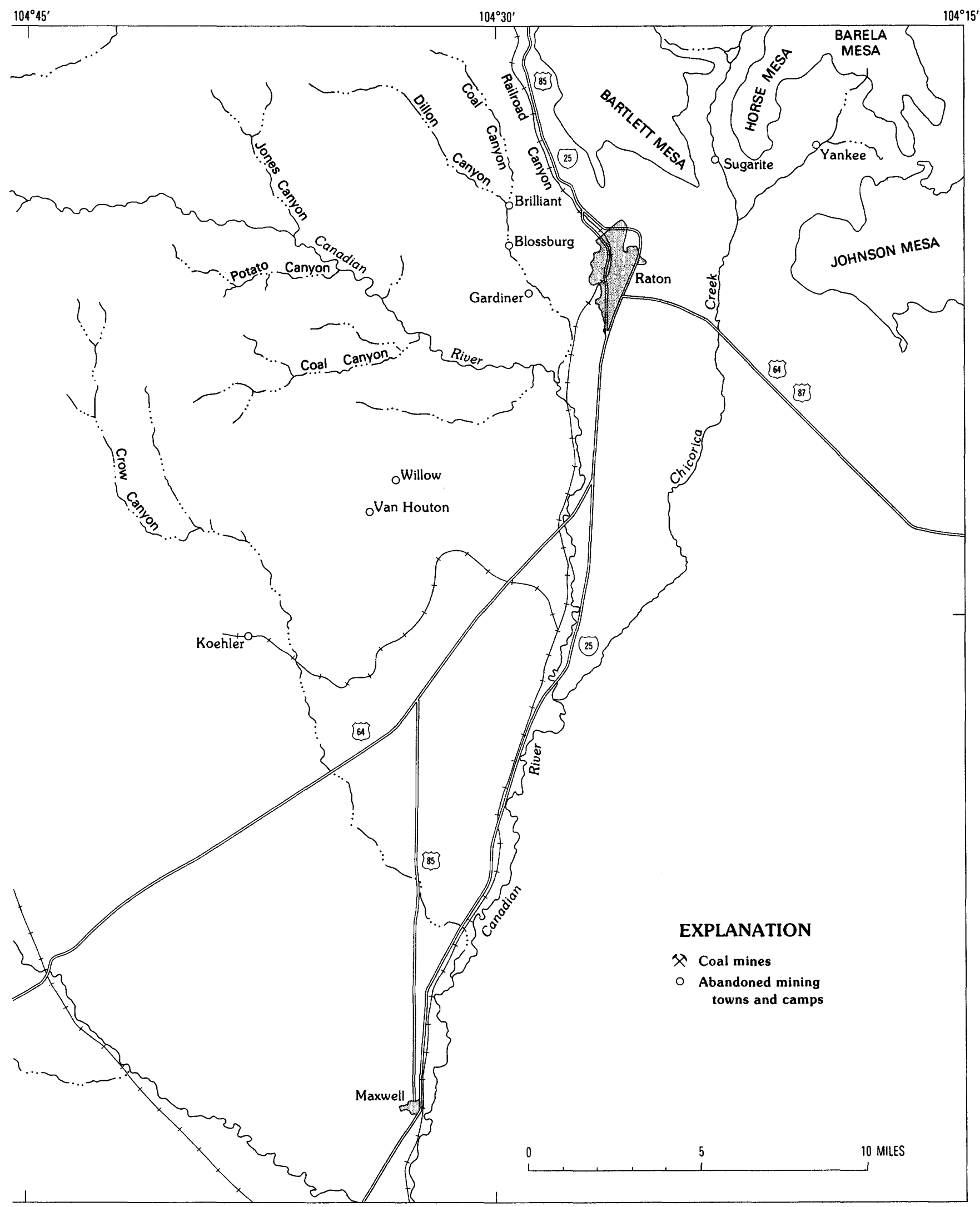

Raton coalfield, New Mexico. 


\section{GEOLOGIC SETTING}

The Raton coalfield lies within the southern half of the Raton basin, a large, arcuate, asymmetric structural basin. This basin is the southernmost of the Laramide intracratonic folded basins at the eastern margin of the Rocky Mountains (Baltz, 1965) and is a sister basin to the San Juan basin, which lies to the west (fig. D2) and contains older but related rocks (fig. D3). The Raton basin extends from near Gardner, Colo., on the north, to Cimarron, N. Mex., on the south-a distance of about $85 \mathrm{mi}$. The structural basin is approximately $70 \mathrm{mi}$ wide at its widest point, along the boundary between Colorado and New Mexico, and narrows both to the north and south. The coalfield is about $50 \mathrm{mi}$ wide at the State boundary. The basin is bounded on the east by the Sierra Grande arch in New Mexico and the Las Animas arch in Colorado. The northern limit is defined by the Apishapa arch (fig. D4). On the west side of the basin, sedimentary rocks of Permian to
Tertiary age are steeply tilted to overturned along the east flank of the Sangre de Cristo Mountains. The axis of the basin, which generally separates more steeply dipping strata from the gently dipping beds to the east, is somewhat sinuous and lies near the west side of the basin.

The Raton coalfield lies entirely in Colfax County, N. Mex. The State line separating the Raton coalfield from the Trinidad coalfield, to the north, nearly coincides with the drainage divide between the Purgatoire River in Colorado and the Canadian River in New Mexico. Coal-bearing rocks in the Raton field underlie a highly dissected plateau whose elevation rises gradually from about $7,000 \mathrm{ft}$ in the eastern part to approximately $9,500 \mathrm{ft}$ in the western part. The coalfield's eastern and southern boundaries, in most places, are marked by nearly horizontally bedded, sandstonecapped escarpments standing several hundred feet above the adjoining high plains. Its western margin is marked by more steeply dipping beds that form hogback ridges and parallel valleys along the eastern front of the Sangre de Cristo

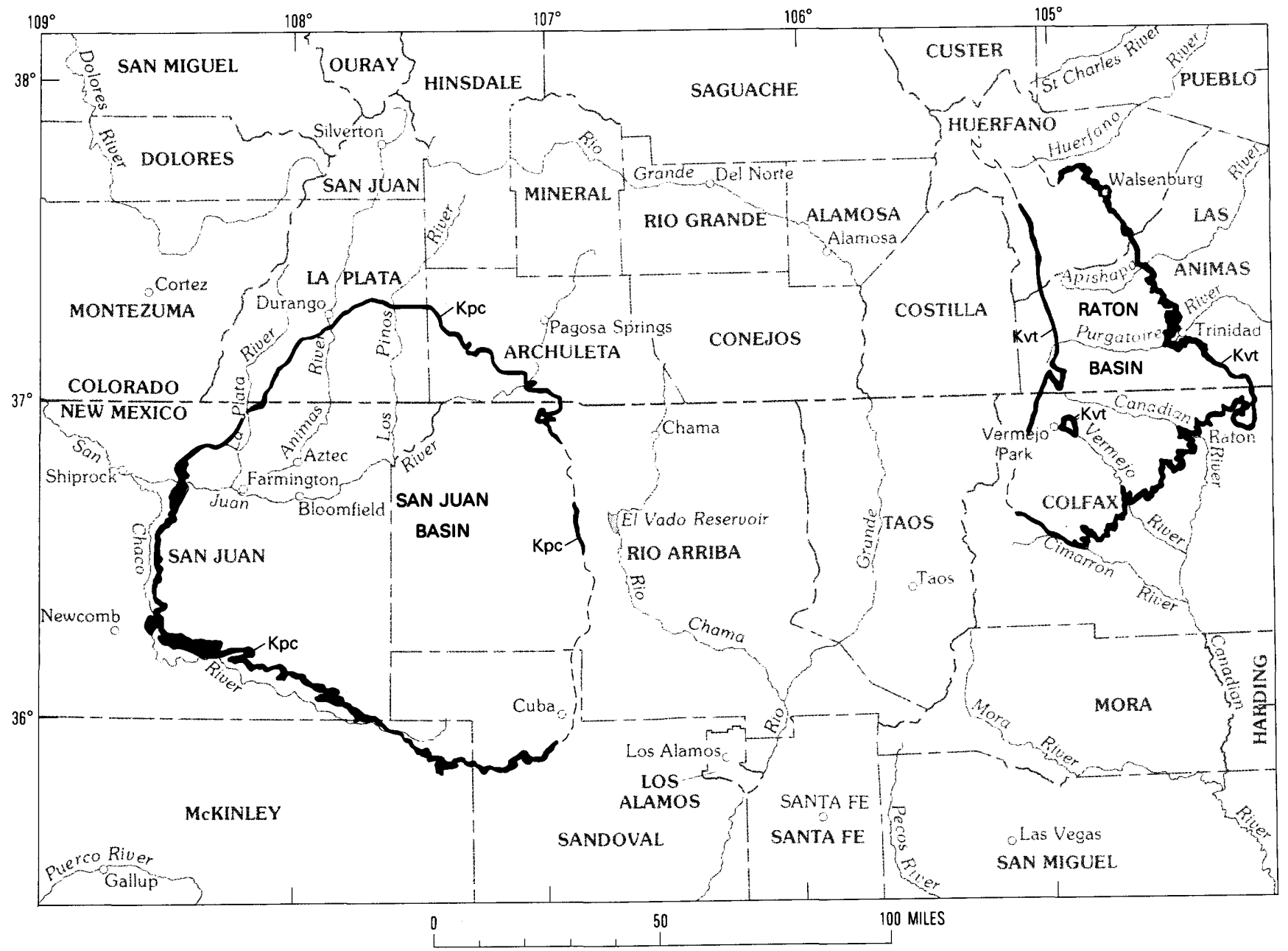

Figure D2. Locations of the Raton and San Juan basins. Kpc, Pictured Cliffs Sandstone; Kvt, Vermejo Formation and Trinidad Sandstone, undivided; all of Late Cretaceous age. (From Fassett, 1976, fig. 1). 


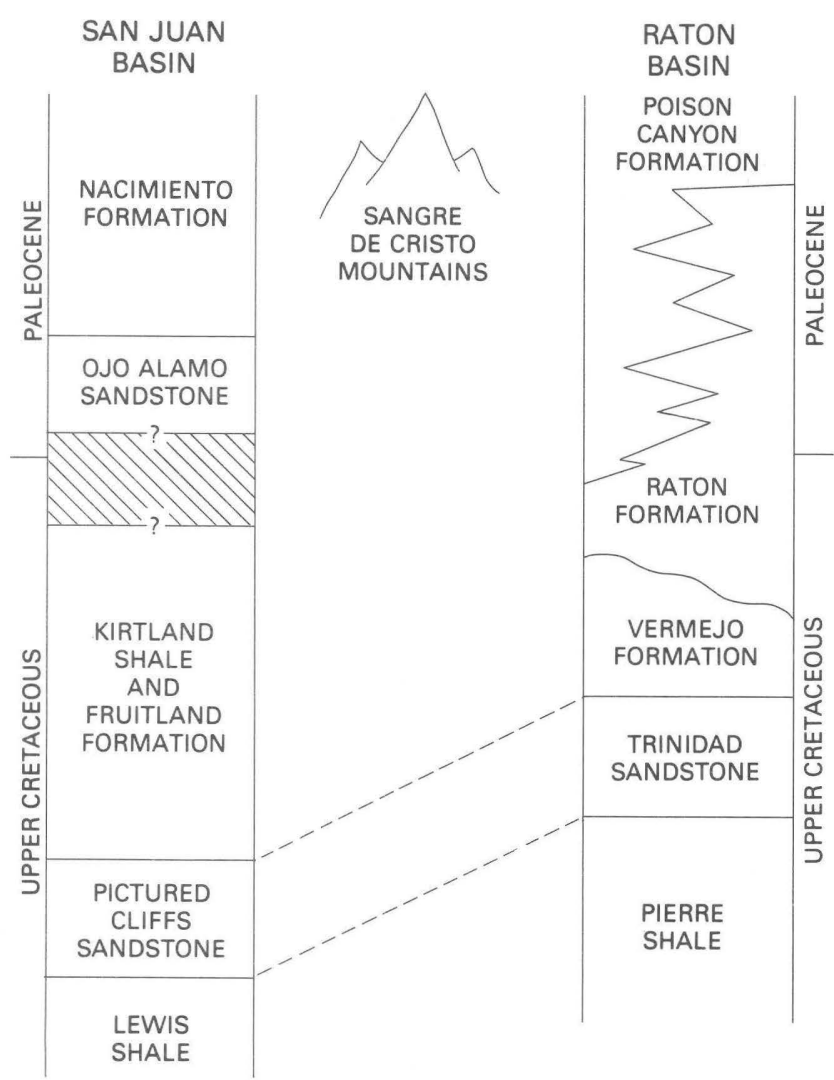

Figure D3. Stratigraphy of Upper Cretaceous and Paleocene rocks of the New Mexico portions of the Raton and San Juan basins.

Mountains. Faults are rarely observed in outcrop, but coal offsets and faults with minor displacement are relatively common features in underground and surface mines in the coalfield. The Vermejo Park anticline, with about $2,500 \mathrm{ft}$ of structural relief, is the only major structure in the coalfield. The position of Vermejo Park and the distribution of the coal-bearing rocks of the Vermejo and Raton Formations are illustrated on the geologic map in figure D5. The cross section in figure D6 shows the anticline, and the stratigraphic sections in the same figure show changes in the Vermejo Formation and some of the principal Raton Formation coal beds.

\section{STRATIGRAPHY}

The stratigraphy of the Upper Cretaceous and Paleocene rocks of the Raton coalfield is shown in table D1, and their relationship with equivalent units in the San Juan basin is shown in figure D3. The offshore-marine Pierre Shale (Campanian to Maastrichtian) and the overlying marginalmarine Trinidad Sandstone (Maastrichtian) underlie the nonmarine Upper Cretaceous and Paleocene rocks in the basin. The position of the Raton basin with respect to the Late Cretaceous epeiric sea is shown in figure D7. Non-

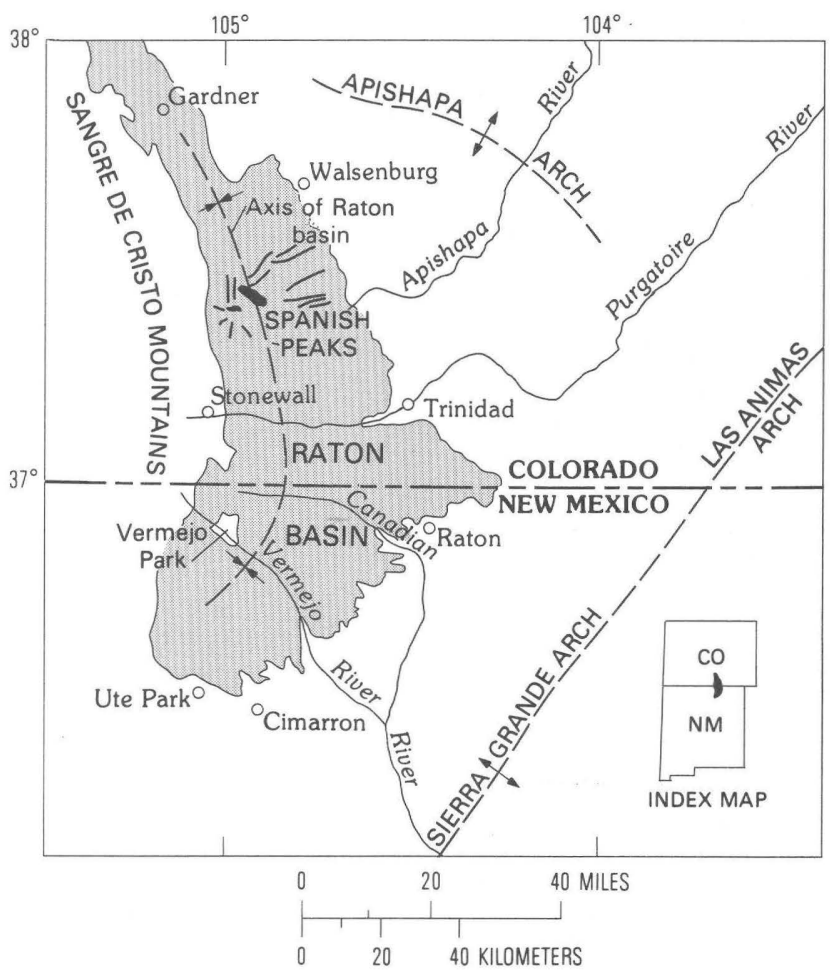

Figure D4. Areal distribution of coal-bearing Upper Cretaceous and Paleocene rocks in the Raton basin. The Raton coalfield is the New Mexico portion of the stippled area.

marine sedimentary rocks in the coalfield, from oldest to youngest, include the coal-bearing Vermejo Formation (Maastrichtian) and Raton Formation (Maastrichtian and Paleocene) and the non-coal-bearing Poison Canyon Formation (Maastrichtian and Paleocene), which overlies and intertongues with the Raton Formation. Much of the following discussion of the rocks and their depositional environments is from Pillmore and Flores (1987).

\section{Pierre Shale and Trinidad Sandstone}

The oldest map unit shown on figure D5 is the Trinidad Sandstone and older rocks unit of Late Cretaceous age. In most parts of the map area, this unit includes only the Trinidad Sandstone and the Pierre Shale. The Pierre formed in prodeltaic and offshore-marine environments and consists of 2,000-2,700 ft of dark, silty, noncalcareous shale. The Pierre Shale grades up into the cliff-forming Trinidad Sandstone, which was deposited in contemporaneous delta-front and interdeltaic barrier-bar environments (Pillmore and Flores, 1987). The Trinidad Sandstone underlies the coal-bearing formations of the Raton coalfield and consists of very fine grained to medium-grained, calcareous to slightly clayey sandstone that ranges in thickness from 0 to $130 \mathrm{ft}$. Zones of abundant Ophiomorpha trace-fossil bioturbation are characteristic of its upper part. 


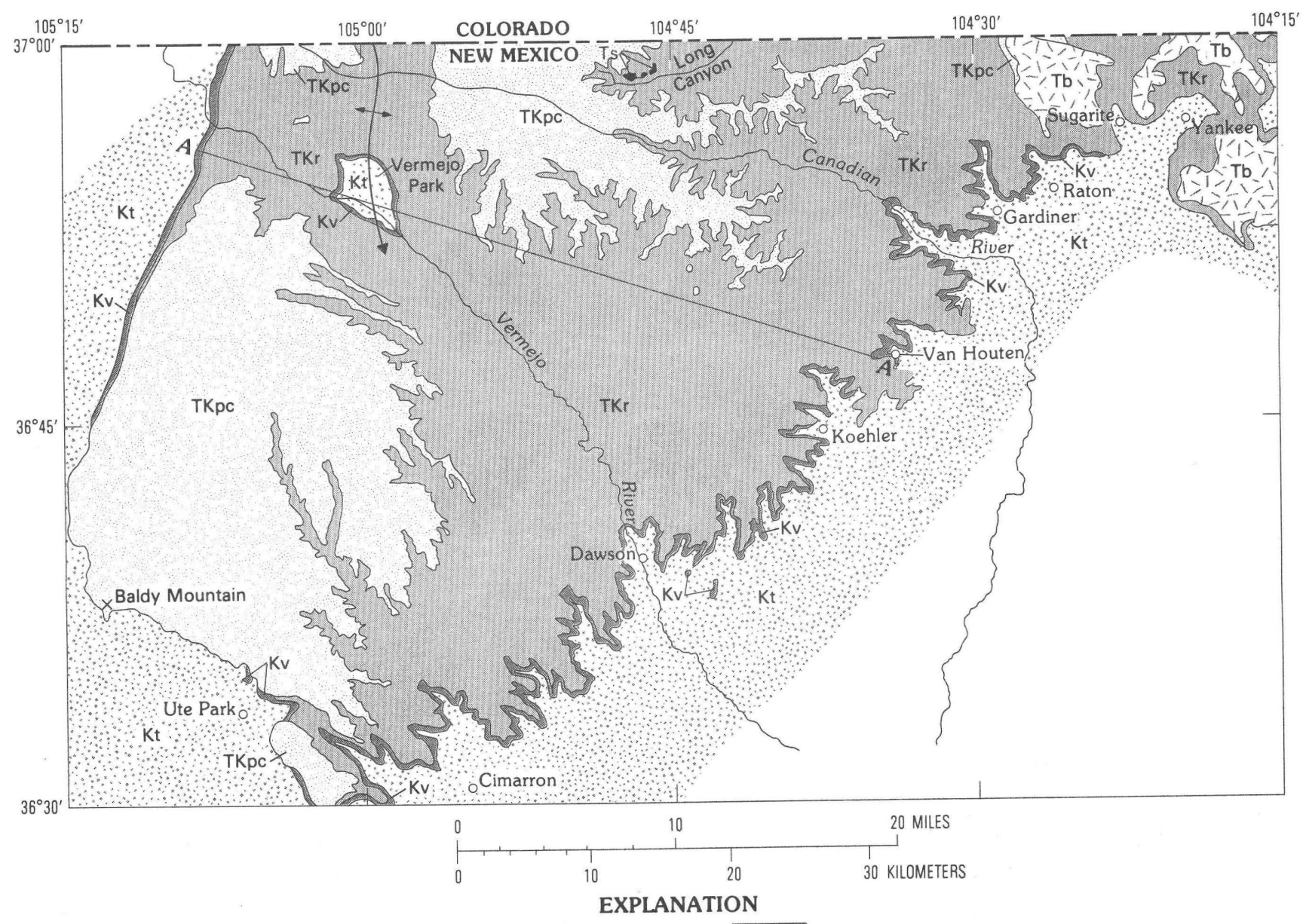

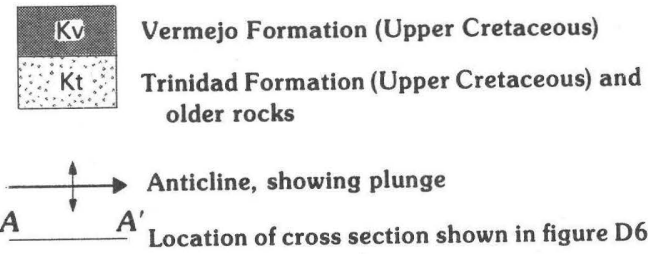

Figure D5. Generalized geology of the Raton coalfield, New Mexico.

\section{Vermejo Formation}

The Vermejo Formation consists of interbedded sandstone, siltstone, shale, carbonaceous shale, and coal that together form steep, generally debris-covered slopes above the cliffs of the Trinidad Sandstone. The Vermejo Formation ranges in thickness from about $375 \mathrm{ft}$ at Vermejo Park and along the western border of the coalfield to $0 \mathrm{ft}$ in the eastern part, east and south of Raton, N. Mex. Coal beds, as thick as $12 \mathrm{ft}$, occur near the top and bottom of the formation. The Vermejo Formation was deposited conformably on the Trinidad Sandstone in contemporaneous fluviodeltaic plain and back-barrier environments. The upper part of the Vermejo Formation in the west-central part of the coalfield was deposited in an alluviated upper delta plain (Flores and Tur, 1982). The
Vermejo Formation intertongues with the Trinidad Sandstone along the southern margin of the coalfield, from a point north of Cimarron northeastward about $11 \mathrm{mi}$ to Dawson, N. Mex. (Wanek, 1963), but this relationship has not been seen in any other areas of the field.

The organic material that ultimately formed the extensive coal beds in the lower part of the Vermejo Formation accumulated in poorly drained, brackish-water swamps or marshes behind barrier islands throughout most of the coalfield and also along the lower reaches of distributary channels of a prograding delta system in the northernmost part of the field. The thick back-barrier coal deposits are aligned subparallel to a north- to northeasttrending, eastward-prograding, Late Cretaceous shoreline, whereas the coal deposits related to the distributary channels are more randomly oriented. Coal beds that formed 
landward, in the upper part of the formation, accumulated in poorly drained backswamps on flood plains of meandering streams in the upper reaches of a fluviodeltaic plain (Pillmore and Flores, 1987).

\section{Raton Formation}

The Raton Formation consists of sandstone, siltstone, mudstone, coal, carbonaceous shale, and conglomerate. It ranges in thickness from more than $2,000 \mathrm{ft}$ in the westcentral part of the coalfield to about $1,100 \mathrm{ft}$ in the eastern part. A basal conglomeratic sandstone bed marks the lower contact and forms a persistent cliff throughout much of the Raton coalfield. Lee (1917) originally divided the Raton Formation into a basal conglomerate, a lower coal zone, a cliff-forming or barren series, and an upper coal zone. However, because the basal conglomerate is absent in the northeastern part of the coalfield, near Raton, it is here included in the lower coal zone, as described fully in Pillmore and Flores (1987). As redefined, this zone contains all of the Cretaceous part of the Raton Formation. Thus only three zones, as modified from Lee (1917), are considered to be consistent and identifiable throughout the Raton basin: the lower coal zone, the barren series, and the upper coal zone.

The lower coal zone, 100-300 feet thick, generally has a basal sequence of ledge-forming, conglomeratic channel sandstone 10-30 ft thick (locally absent), which grades up into slope-forming, overbank, flood-plain deposits. The flood-plain deposits consist of interbedded sequences of mudstones, siltstones, carbonaceous shales, and thin coal beds (mostly noncommercial) interbedded with lenticular channel and tabular crevasse-splay sandstones. The tabular crevasse-splay sandstones show rippled and small-scale cross lamination. The carbonaceous shale beds and thin coal beds were deposited in well-drained backswamps that formed repeatedly and expanded over the flood plain during nonflood conditions. However, in the eastern part of the coalfield, about 3 mi northeast of Raton, poorly drained swamp conditions existed over a large area (approximately $30 \mathrm{mi}^{2}$ ) for a prolonged time at the end of the Cretaceous. Peat deposits accumulated in this swamp, nearly uninterrupted by detrital deposits, to form a 6-ft-thick coal bed known as the Sugarite coal bed at the top of the lower coal zone of the Raton Formation. The palynological break that defines the Cretaceous-Tertiary (K-T) boundary occurs near the top of the coal bed in a kaolinitic, iridiumenriched claystone bed that apparently formed as a result of asteroid impact (Pillmore and Flores, 1987). This K-T boundary claystone bed has been found at several sites in the Raton coalfield and at other sites as far north as Alberta, Canada. It is useful as a chronostratigraphic marker.

The barren series, $175-600 \mathrm{ft}$ thick, is dominated by cliff-forming, multiple-stacked, en echelon channel sandstones that are mostly fine to medium grained. The channel sandstones are nested to form a series of ledges and cliffs, but they are locally interbedded with and laterally grade into minor flood-plain deposits of mudstone and siltstone. Associated backswamp carbonaceous shale and coal beds are mostly thin and limited in extent.

The upper coal zone, $600-1,100 \mathrm{ft}$ thick, consists of flood-plain deposits of sandstone, mudstone, siltstone, and carbonaceous shale interbedded with thick beds of coal, which represent occasional prolonged backswamp accumulations of peat. Coal beds in the upper coal zone are generally thicker and less restricted than those in the lower coal zone; however, most are lenticular and less than $3 \mathrm{ft}$ thick. The upper zone includes several commercial beds, attaining thicknesses of $10 \mathrm{ft}$ or more, and many are considered economically minable. The flood-plain and backswamp deposits of the upper coal zone are interbedded with crevasse-splay and channel sandstones.

\section{Poison Canyon Formation}

The Poison Canyon Formation overlies the Raton Formation over most of the eastern part of the basin but to the west and southwest it intertongues with all zones of the Raton and thickens at the expense of the Raton. In this area the Poison Canyon typically consists of thick to massive, lenticular, ledge-forming beds of coarse-grained to conglomeratic arkosic channel sandstone intercalated with beds of nonresistant, yellowish-gray- to grayish-orangeweathering, sandy, micaceous mudstone. These finegrained rock types represent overbank flood-plain deposits. They grade laterally into and are interbedded with the channel sandstones. The Poison Canyon Formation is barren of coal and is interpreted as deposits of braided streams in an upper alluvial plain.

\section{Igneous Rocks}

Igneous flows, dikes, and sills, although not shown in figure D5, crop out in many places in the Raton coalfield. Basalt flows 3.5-4.0 m.y. old (Scott and Pillmore, 1989; Scott and others, 1990) cap the mesas along the northeastern border of the field, and lamprophyric dikes of mafic to intermediate composition, mostly about 25 m.y. in age (Scott and Pillmore, 1989) are found in almost all parts of the field. The dikes generally caused little alteration of the intruded beds; in contrast, the sills commonly intruded and stoped along coal beds, especially in the Raton coal zone of the Vermejo Formation, and have assimilated, coked, altered, and destroyed much coal (Pillmore, 1976). It is not known if the coking character of the coal beds in the Raton coalfield can be attributed to heat from igneous activity at depth. 


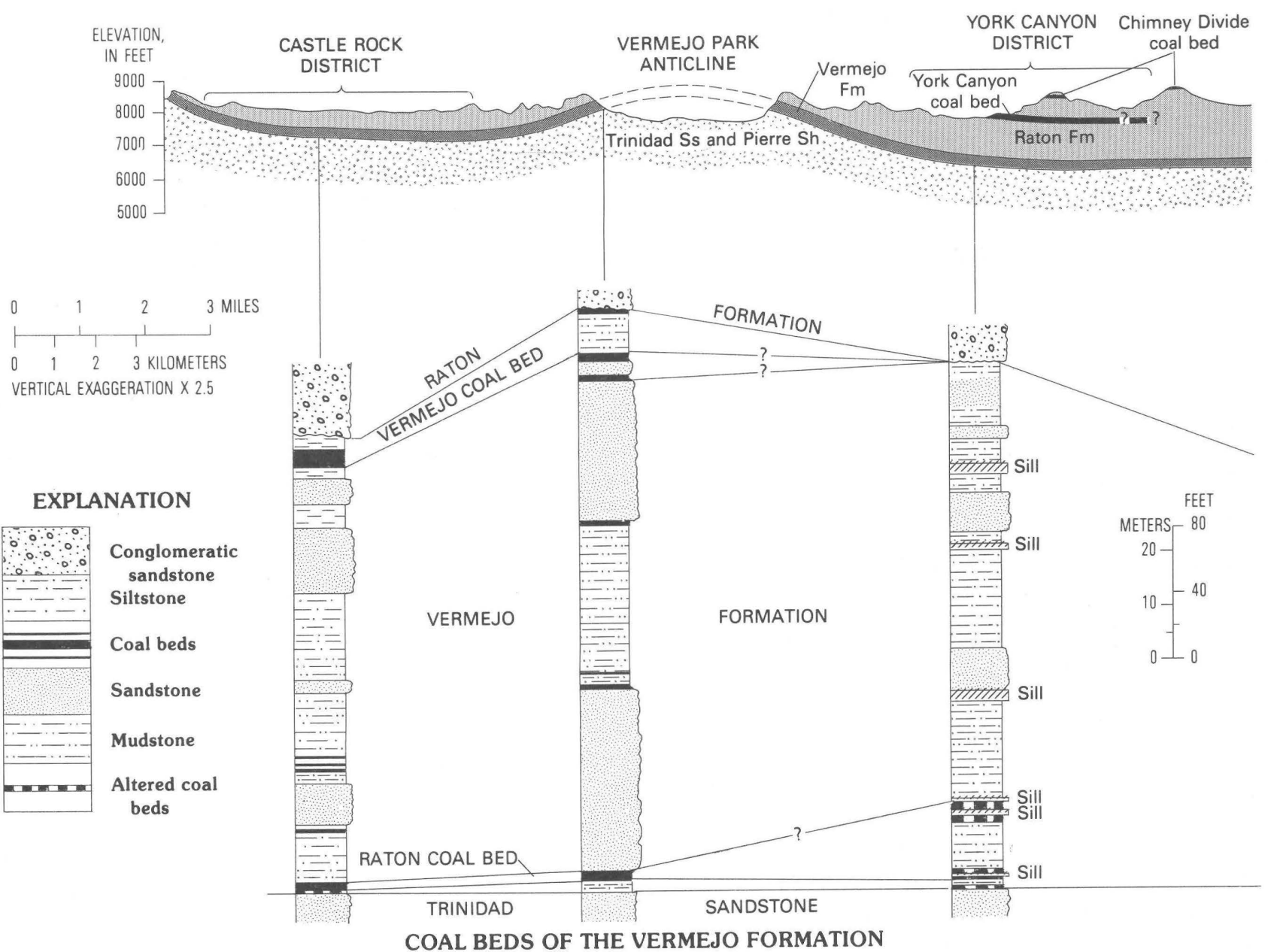

Figure D6. Diagrammatic geologic cross section and columnar sections showing Vermejo Park anticline and lithologic shown on figure D5. The cross section is extended diagrammatically to the east to show the thinning of the Vermejo Formation.

\section{COAL RESOURCES}

The Raton basin contains modest coal resources compared to some coalfields now being prospected, developed, and mined elsewhere in the western United States; however, the coal from the Raton field has high heating values, is of high rank, yields a high-quality coke, and may be of greater economic importance than coal from other larger fields. The coal is all high-volatile A to B bituminous rank; some representative coal analyses are given in table D2, and additional data on volatile matter and sulfur are shown in table D3 (Pillmore, 1976).

The Raton coalfield is unusual in western coal lands in that it consists almost entirely of fee land, where domestic energy companies have owned and developed the mineral rights over a long period. Under these circumstances, the buried coal resources of the field have not been thoroughly evaluated by either the State or the Federal Government. However, various field surveys and surface investigations have been carried out, beginning with the earliest territorial surveys and work by Orestes St. John in the late 1800's (St.
John, 1896), followed by W. T. Lee's investigations in the early 1900's (Lee, 1917). The U.S. Geological Survey (USGS) began investigations in the coalfield again in the mid-1900's (Wanek, 1963; Pillmore, 1969a and b, 1976), and since that time the work has been sporadic. The underground resources of large parts of the field have been explored by drilling and evaluated by Kaiser Steel Corporation (1954-84), Kaiser 'Coal Corporation (1984-89), and, most recently, by P\&M Coal Mining Company (1989-present). These companies have provided much useful information but have asked not to have specific drilling sites and depth and thickness data published.

Coal resources for the various districts in the Raton coalfield are listed as demonstrated resources in table D4 (Pillmore, 1976). The demonstrated category includes measured and indicated resources calculated by standard techniques from drill hole data and limited outcrop information. P\&M Coal Company prefers not to release resource information in any more detailed resource categories. The company's tonnage calculations are based on 85 $\mathrm{lbs} / \mathrm{ft}^{3}$ or 1851 tons/acre $\mathrm{ft}$ for bituminous coal in place, as 


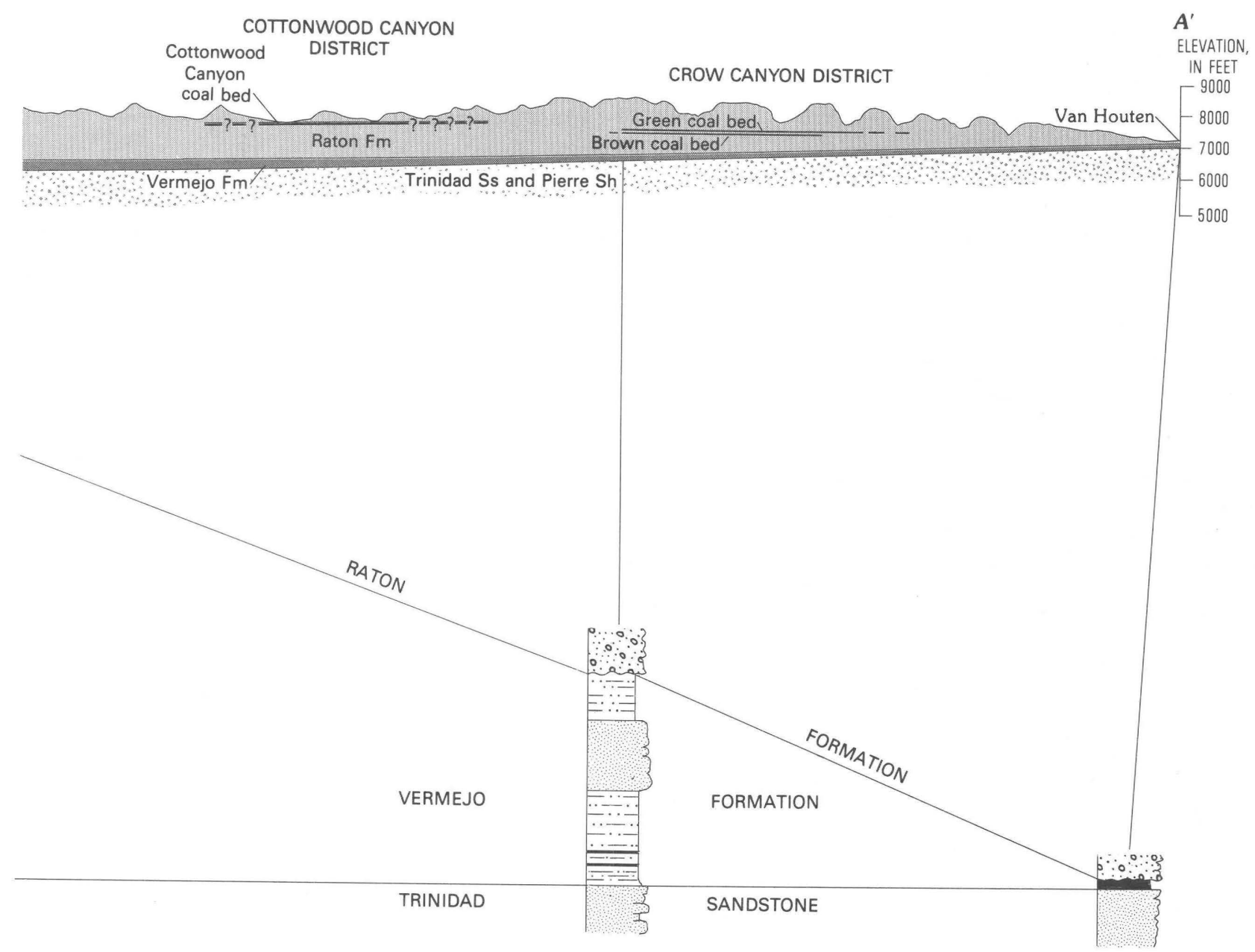

changes and coal beds in the Vermejo Formation across the northwestern part of the Raton coalfield. Trace of cross section

compared to 1800 tons/acre $\mathrm{ft}$, which is the figure commonly used by the U.S. Geological Survey (Wood and others, 1983) to calculate bituminous coal in place.

Coal beds of potential commercial interest-here considered to be those beds at least 30 in. thick-occur throughout much of the area that is underlain by coalbearing rocks. However, many of the beds are of limited extent, and available data are too sparse to allow the beds to be considered separately in this report. The most extensive and valuable deposits are the Raton and Vermejo coal beds, in the Vermejo Formation, and a group of coal beds that include the York Canyon and Upper Left Fork coal beds, presently being mined, in the upper coal zone of the overlying Raton Formation. The distribution of these formations is shown on the geologic map (fig. D5). The areas underlain by the beds of former, present, or future commercial importance are here informally called coal districts, and the most important of these districts are shown on figure $\mathrm{D} 8$.

Both formations also contain beds less than 30 in. thick. Demonstrated resources have not been calculated for these beds and they are listed here as inferred resources. To show a rough distribution of these inferred resources, the coalfield has been arbitrarily divided into 15 geographic subdivisions (fig. D9), which overlap with but are distinct from the districts defined for the demonstrated resources (fig. D8). Minimum total coal thicknesses in both the Vermejo and Raton Formations have been estimated for each of the various subdivisions that make up the Raton coalfield. In each subdivision, drilling records, electric logs, and outcrop data were examined and interpreted, and a value representing the minimum total coal thickness of beds 14 in. thick or greater was assigned for each formation (table D5). The numbers are based on geologic subsurface and surface data, but the values are subjective in that no statistical approach was used for their generation. The resources are listed as inferred rather than hypothetical because of the large amount of data available throughout much of the coalfield; however, estimates for the southern part of the coalfield (the Ponil and Lower Bremmer subdivisions) are less reliable than those for other parts because drill holes there are more widely spaced. When 


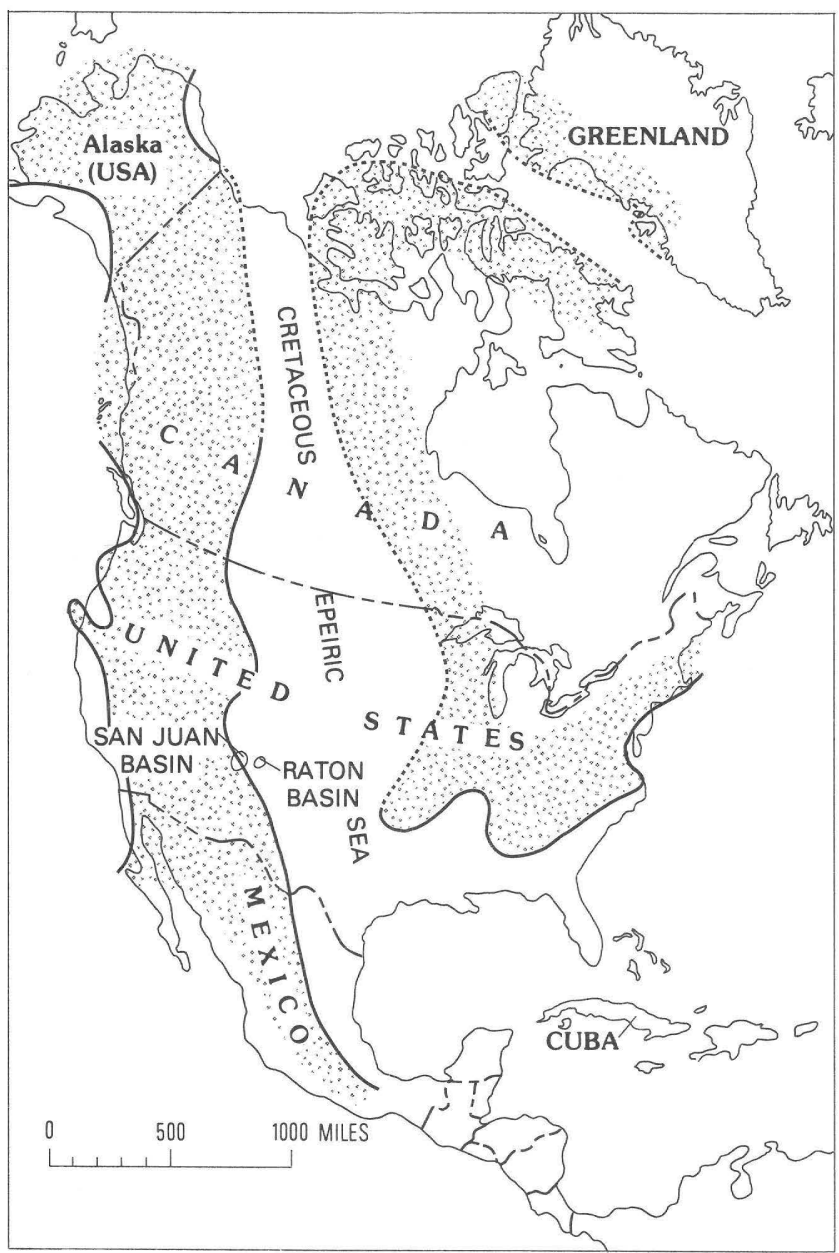

Figure D7. Western Interior seaway during Late Cretaceous time, showing the locations of the Raton and San Juan basins. (Modified from Gill and Cobban, 1966, fig. 15.)

these inferred resources (table D5) are added to the demonstrated resources calculated for the districts (table D4), the total gives a rough indication of the potential coal resources of the entire coalfield.

\section{Coal Deposits in the Vermejo Formation}

As shown in figure D6, the principal economic coal beds of the Vermejo Formation are the Raton coal bed (not to be confused with the formation of the same name), which lies at the base, and the Vermejo coal bed, which is near the top in the west-central part of the coalfield and is shown in the sections at Castle Rock and Vermejo Park. Although other coal beds as thick as 3-4 ft occur in the Vermejo, only these two are known to have sufficient extent and thickness to be considered potentially economic. The most economically important districts, defined by coal deposits in the Vermejo Formation that have not yet been mined, are Castle Rock and Rosado (fig. D8).
The Raton coal bed underlies most of the Raton coalfield. However, subsurface investigations indicate large areas where much of the coal has been altered or destroyed by sills that have intruded the Raton coal bed. In some areas, the presence of sills is known only from isolated drill holes and the extent of intrusive activity is uncertain. Several large districts in the coalfield are defined by the presence of thick coal in the coal zone on or immediately above the Trinidad Sandstone. Most of these districts have been extensively mined and, because only sketchy records remain, the areal extents of these thick coal beds are uncertain. The mines that saw most of the early activity are those at or near the abandoned mining towns of Gardiner, Brilliant, Willow, Van Houten, Koehler, and Dawson, and they are essentially worked out. (The Brilliant district, as shown in fig. D8, is a north and northwest extension of the old Gardiner and Brilliant districts.) Coal deposits of these old districts are described by Lee $(1917 ; 1924)$.

\section{Castle Rock District}

The Castle Rock district contains the largest known coal deposits in the coalfield. The Raton and Vermejo coal beds underlie about 25,000 acres, or nearly $40 \mathrm{mi}^{2}$, in the Castle Rock district (fig. D8). This large district extends from the western margin of the coalfield about $4 \mathrm{mi}$ eastward, nearly to Vermejo Park, and has been traced in a north-south direction for about $9 \mathrm{mi}$. Along the western margin of the district the rocks dip eastward as steeply as $25^{\circ}-30^{\circ}$. In the areas considered minable, the stratigraphic distance from the Trinidad Sandstone to the Raton coal bed ranges from 5 to $35 \mathrm{ft}$ (mostly 10-15 ft or less); the Vermejo coal bed lies about $250 \mathrm{ft}$ above the Raton coal bed. The coal beds range in thickness from 3 to $15 \mathrm{ft}$ and constitute a demonstrated coal resource containing more than 500 million tons (table D4). The overburden ranges from 0 along the outcrop to more than $3,000 \mathrm{ft}$ below the high mesas in the southwestern part of the bed area. The Raton coal bed underlies fine-grained rock sequences consisting mostly of mudstone and shale. The Vermejo coal bed also mostly underlies fine-grained rocks, but locally the basal conglomerate of the Raton Formation rests directly on the coal bed.

According to corporate mining experts, problems related to roof rock and depth of cover in the Castle Rock district can be solved by the use of yieldable arches and modern longwall mining methods; however, the economics of using these methods will be the chief factor controlling the development of this district. Mining problems would be more severe in the areas of steeply dipping coal seams, along the western margin of the coalfield, but the rocks dip more gently in most parts of the district, and mining is not expected to be too difficult. 
Table D1. General stratigraphic description of the Raton coalfield, New Mexico

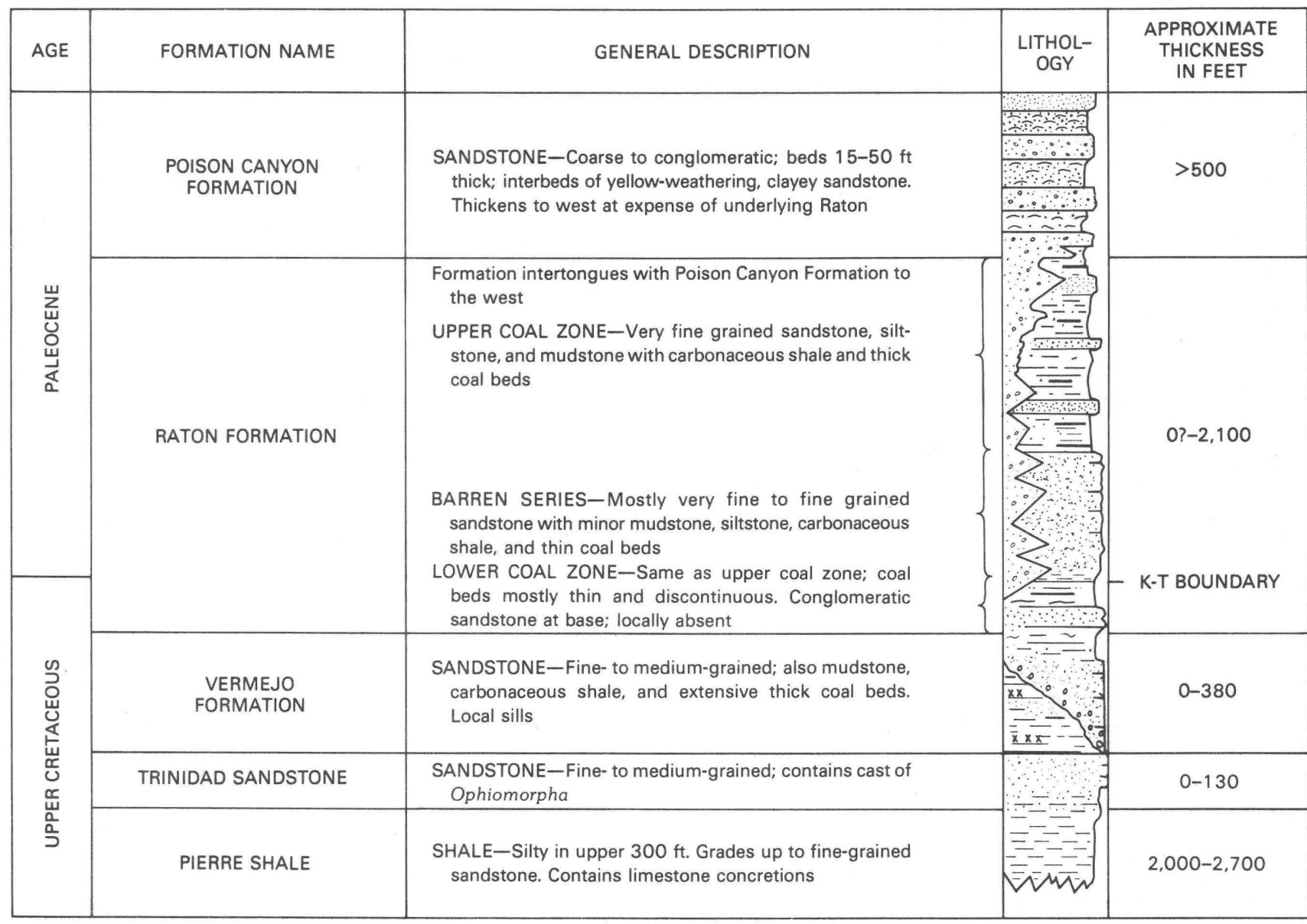
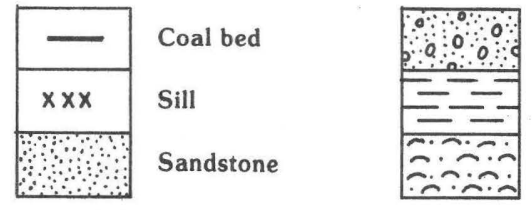

\section{EXPLANATION}

Conglomeratic sandstone

Shale

Clayey sandstone

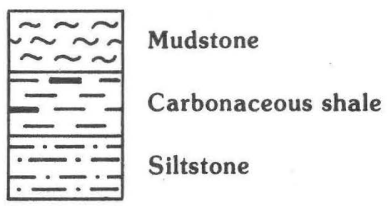

\section{Rosado District}

South of Vermejo Park, in the Rosado district, the Raton and Vermejo coal beds underlie an area of about 9,500 acres, mainly in Juan Baca Canyon. These beds constitute a demonstrated coal resource of about 127 million tons (table D4) and appear to form an eastward extension of the Castle Rock district, though the continuation of these beds from one district to the other is uncertain. The Vermejo coal bed is present throughout most of the district and constitutes the greater part of the resource. Locally, the basal conglomerate of the Raton Formation rests directly on the coal, but in most parts of the district it lies $15-30 \mathrm{ft}$ above the coal bed. The beds dip south about $10^{\circ}-15^{\circ}$ along the south rim of Vermejo Park, south of the Vermejo Park anticline, but dips flatten rapidly away from the structure.
Entries developed in the Vermejo coal bed in the Rosado district, on the southeast flank of the Vermejo Park anticline, could provide access to the large underground resources of the Castle Rock district to the west of Vermejo Park. Exploitation of the Castle Rock district from the Rosado district might prove to be less disruptive environmentally than any other approach.

The Raton and Vermejo coal beds also crop out on the flanks of the Vermejo Park anticline and underlie the area north of Vermejo Park. These areas do not appear to contain resources sufficient to define a separate district. At the old Bartlett (Dead Easy) mine, at the north entrance to Vermejo Park, the Vermejo coal bed is about $6 \mathrm{ft}$ thick, and coal was mined for several years for local ranch use. Both coal beds locally reach a thickness greater than $8 \mathrm{ft}$ at outcrops around 
Table D2. Representative proximate analyses of selected coal beds in the Raton coalfield, New Mexico

[Data in percent, except for Btu]

\begin{tabular}{|c|c|c|c|c|c|c|c|}
\hline Mine or location & Coal bed & $\begin{array}{c}\text { Volatile } \\
\text { matter }\end{array}$ & $\begin{array}{l}\text { Fixed } \\
\text { carbon }\end{array}$ & Ash & sulfur & Btu & $\begin{array}{l}\text { Source } \\
\text { of data }\end{array}$ \\
\hline \multicolumn{8}{|c|}{ Raton Formation } \\
\hline York Canyon mine - & York Canyon --- & 35.7 & 54.9 & 8.8 & 0.6 & 14,340 & 1 \\
\hline Chimney Divide --- & Chimney Divide & 38.4 & 47.6 & 13.4 & .5 & 13,980 & 1 \\
\hline Ancho Canyon -..-- & Ancho Canyon -- & 36.3 & 54.0 & 8.6 & .5 & 13,835 & 1 \\
\hline \multicolumn{8}{|c|}{ Vermejo Formation } \\
\hline Vermejo Park _...- & Vermejo ------- & 28.2 & 53.7 & 18.0 & 0.5 & 10,030 & 3 \\
\hline & Raton - & 25.4 & 60.5 & 14.1 & .5 & 11,440 & 3 \\
\hline Dawson (No. 3) & -- do ------ & 38.2 & 49.3 & 12.5 & .8 & 13,230 & 2 \\
\hline
\end{tabular}

\section{SOURCES OF DATA}

1. Bulk core sample data courtesy of Kaiser steel Corporation. Btu results obtained from coal washed in heavy liquid (specific gravity 1.4).

2. Mine analysis, moisture free, selected from Ellis (1936, p. 44-48).

3. Weathered outcrop sample analysis courtesy of U.S. Bureau of Mines (generally higher ash and lower calorific values); low volatile content probably caused by intrusive rocks.

Table D3. Ranges of volatile matter and sulfur in 164 core samples of the major coal beds in the Raton coalfield, New Mexico

[Moisture- and ash-free basis. Data from whole-bed core samples washed in a heavy liquid (specific gravity about 1.5 ). Samples included partings and coal splits, so aggregate values represent the character of coal that would result from a beneficiation process. Data courtesy of Kaiser Steel Corporation, 1976]

\begin{tabular}{|c|c|c|c|}
\hline $\begin{array}{l}\text { Sample locality } \\
\text { and coal bed }\end{array}$ & $\begin{array}{l}\text { Number } \\
\text { of } \\
\text { samples }\end{array}$ & $\begin{array}{c}\text { Volatile } \\
\text { matter } \\
\text { (percent) }\end{array}$ & $\begin{array}{l}\text { Sulfur } \\
\text { (percent) }\end{array}$ \\
\hline \multicolumn{4}{|l|}{ York Canyon mine area: } \\
\hline \multicolumn{4}{|l|}{ Cimarron mine area: } \\
\hline Upper Left Fork bed & 17 & $35.6-40.1$ & $.44-.56$ \\
\hline Lower Left Fork bed & 10 & $37.4-41.0$ & $.48-.57$ \\
\hline \multicolumn{4}{|l|}{ Castle Rock area: } \\
\hline Raton bed & 41 & $29.2-42.5$ & $.60-.90$ \\
\hline Vermejo bed ............ & 34 & $32.4-41.9$ & $.63-.93$ \\
\hline \multicolumn{4}{|l|}{ Central area: } \\
\hline Ancho bed & 21 & $43.1-46.1$ & $.50-.65$ \\
\hline Green bed ............. & 18 & $40.6-45.4$ & $.46-.69$ \\
\hline Brown bed & 9 & $37.2-39.8$ & $.45-.55$ \\
\hline Potato Canyon bed -- & 6 & $39.6-44.0$ & $.51-.74$ \\
\hline
\end{tabular}

the perimeter of Vermejo Park, but the beds seem less extensive than in the Castle Rock district, and sills probably have intruded the coal locally. The extent of the coal beds north from Vermejo Park is unknown.

\section{Brilliant District}

The Brilliant district is north and northwest of Raton (fig. D8). It consists of about 16,000 acres in the area drained by Dillon Canyon and its tributary, Coal Canyon, and in the part of the Railroad Canyon drainage west of Interstate Highway 25. Coal was mined extensively from the Raton coal bed in the old Blossburg mining district (not shown on fig. D8), which is south of the Brilliant district and included the mines at Blossburg, Gardiner, and Brilliant in Dillon Canyon. Undisturbed coal resources in the Raton coal zone have been defined by subsurface investigations in the Brilliant district, mainly north and northwest of the old mines. Potentially economic coal beds extend also under the area east of Interstate 25, redefined as the Brilliant East subdistrict. Demonstrated coal resources in the Brilliant district total 79 million tons (table D4 in beds of the Raton coal zone. The Raton coal zone locally consists of two or three coal beds separated by partings as much as $10 \mathrm{ft}$ thick; the coal beds in the zone range in thickness from 3 to $8 \mathrm{ft}$ (Lee, 1924). The Brilliant district is potentially economic because of the high quality of its coal and its close proximity to Raton and to rail transportation in Railroad Canyon.

Brilliant East subdistrict.-The Brilliant East subdistrict includes roughly 5,000 acres east of Interstate 25, as shown on figure D8. The eastward extent of the Raton coal zone beneath the basalt cap of Bartlett Mesa is uncertain (fig. D5); however, the Raton coal zone is known to be 
Table D4. Demonstrated coal resources of commercial thickness (>30 in.) within mining districts of Raton coalfield, New Mexico

[Courtesy of Kaiser Coal Corporation]

\begin{tabular}{|c|c|c|c|c|}
\hline District & $\begin{array}{c}\text { Approx- } \\
\text { imate } \\
\text { area } \\
\text { (acres) }\end{array}$ & $\begin{array}{l}\text { Coal bed } \\
\text { or zone }\end{array}$ & $\begin{array}{c}\text { Average } \\
\text { coal } \\
\text { thick- } \\
\text { ness } \\
\text { (feet) }\end{array}$ & $\begin{array}{c}\text { Demon- } \\
\text { strated } \\
\text { resour- } \\
\text { ces } \\
\left(10^{6}\right. \\
\text { tons) }\end{array}$ \\
\hline
\end{tabular}

\begin{tabular}{|c|c|c|c|c|}
\hline \multicolumn{5}{|c|}{ Resources in the Vermejo Formation } \\
\hline Castle Rock -- & 25,600 & 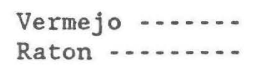 & $\begin{array}{l}5.5 \\
6.0\end{array}$ & $\begin{array}{l}250 \\
270\end{array}$ \\
\hline Rosado ....... & 9,500 & $\begin{array}{l}\text { Vermejo } \\
\text { Raton }\end{array}$ & $\begin{array}{l}5.5 \\
4.0\end{array}$ & $\begin{array}{r}102 \\
25\end{array}$ \\
\hline Brilliant ...- & 4,200 & -. do ........ & 5.0 & 79 \\
\hline Brilliant East & 3,200 & - do & 5.0 & 30 \\
\hline Koehler ${ }^{1} \ldots$. & 4,500 & -. do & 5.0 & 19 \\
\hline Dawson $^{2}$ & 11,200 & - do.......- & 3.0 & 196 \\
\hline Vermejo For & mation $t$ & otal ...... & - . - - & 971 \\
\hline
\end{tabular}

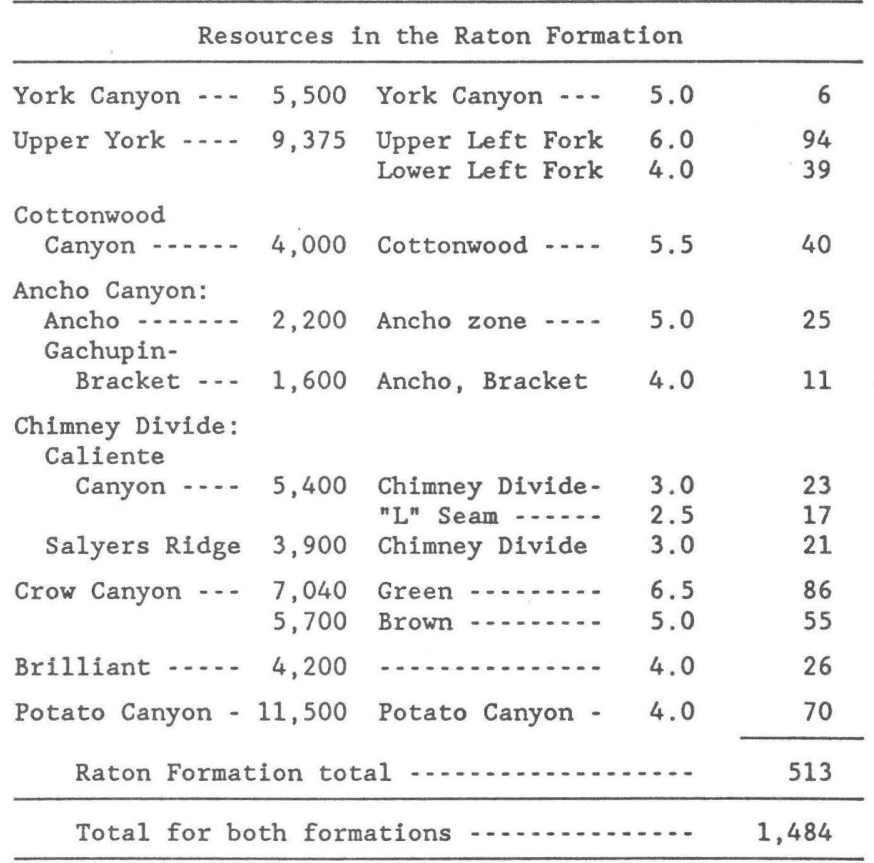

${ }^{1}$ Partly mined out; remaining tonnage estimated. ${ }^{2}$ Modified from Wanek (1963).

present, and 30 million tons of demonstrated resources were assigned to this subdistrict following preliminary subsurface evaluation.

\section{Dawson and Koehler Districts}

Coal was produced from the coal mines at Dawson from the late 1800's until 1950, and at Koehler from 1906 until 1966, when production was started at York Canyon. The Koehler district (fig. D8) produced 14 million tons of coal and still contains demonstrated resources of 19 million tons (table D4). The Dawson district (fig. D8), owned by Phelps Dodge Corporation, produced 33 million tons of coal between 1899 and 1950 and surely has large reserves remaining. Some drilling has been done in the district in recent years, but the resulting data are not available. Wanek (1963) calculated demonstrated resources of 196 million tons of coal in beds of the Vermejo Formation in the Dawson district, mainly based on surface investigations. The district is of interest because of its relatively easy access to transportation (the Santa Fe Railway crosses the district in the Vermejo River canyon) and possible outcrop mine entries.

\section{Other Areas}

The intrusion of sills appears to have destroyed much of the coal in the Vermejo Formation in areas east of Vermejo Park and along the Vermejo River south of the Ancho subdistrict (fig. D8). During the summer of 1989, a large area in Van Bremmer Canyon was the focus of heavy drilling activity in the search for coal-bed methane by Pennzoil Corporation. Preliminary evaluation of the data indicates the presence of significant coal beds in the Vermejo Formation and also suggests that the Castle Rock and Vermejo Park-Rosado thick coal trends possibly extend south into this area.

In most districts of the coalfield, both the Vermejo and Raton Formations contain thin, local coal beds in addition to those thicker, more extensive beds that define the districts. Coal resources in the Vermejo Formation in beds $14 \mathrm{in}$. thick or greater are inferred to underlie the subdivisions of the coalfield shown on figure D9. The resources listed in table D4 are based on geologic inference and represent a minimum estimate of inferred coal resources contained in both the Vermejo and Raton Formations. The estimate for the Upper Bremmer-Gachupin and Lower Bremmer subdivisions are probably low, based on the new Pennzoil drilling data.

\section{Coal Deposits in the Raton Formation}

A large part of the coal resource of the Raton coalfield is in coal beds of the Raton Formation. As described above, these beds were formed in poorly drained swamps on flood plains developed along meandering streams of a broad, latest Cretaceous and early Paleocene alluvial plain. Because of their fluvial setting, these swamps were not as extensive as the back-barrier swamps that formed the Raton coal bed. The proximity of the Raton basin at this time to an actively uplifting source area, the San Luis Highland (Tweto, 1980), usually precluded the prolonged stable conditions that would have been needed to create widespread thick accumulations of peat and, hence, widespread thick coal deposits. However, multiple beds 


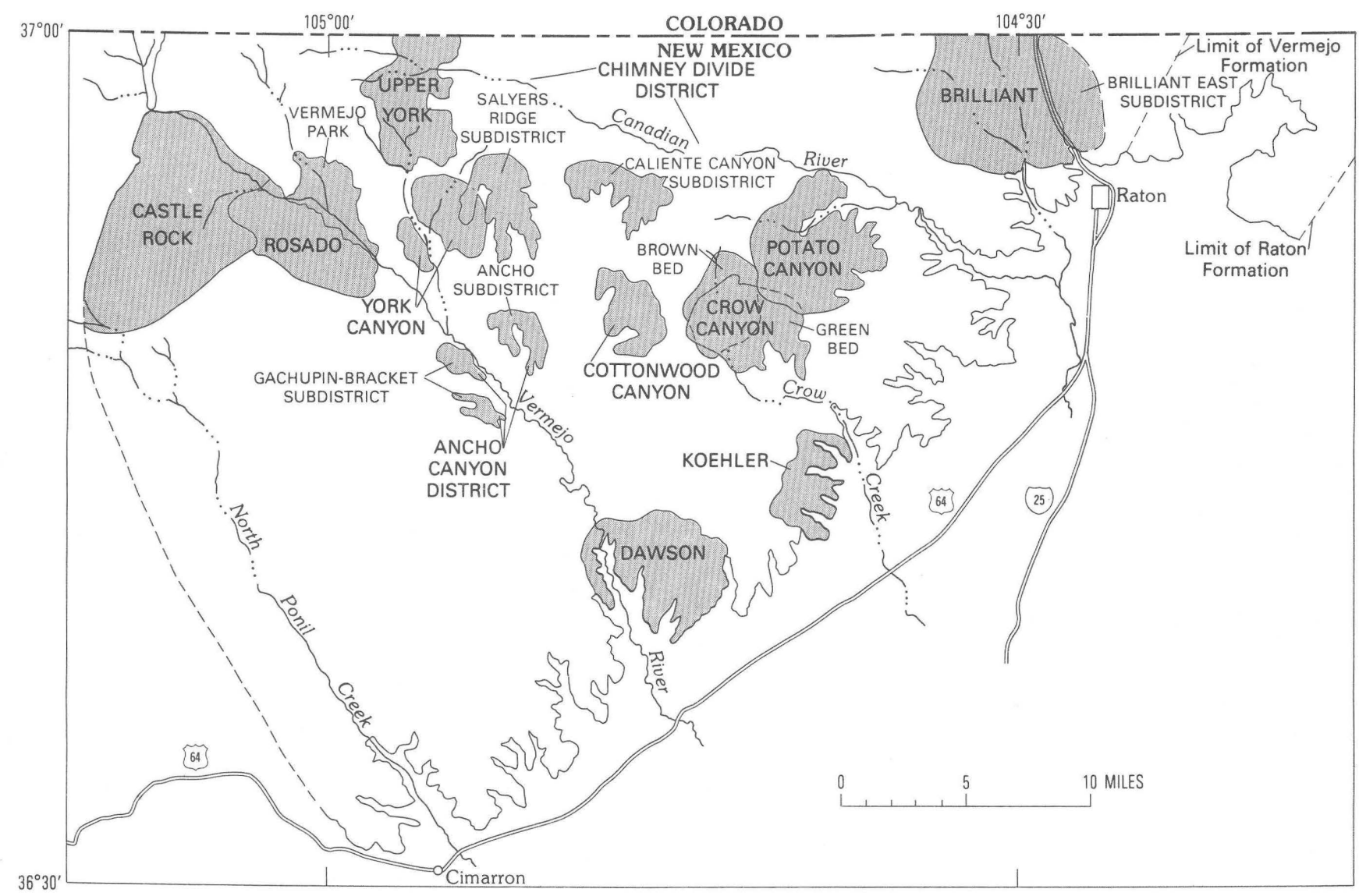

Figure D8. Districts of the Raton coalfield, New Mexico, that have demonstrated coal resources, as outlined by subsurface exploration.

ranging in thickness from 2 to $4 \mathrm{ft}$ together with the thicker beds that define the districts constitute a viable resource in the coalfield. Many areas underlain by the upper coal zone of the Raton contain aggregate coal thicknesses in excess of $15 \mathrm{ft}$, in beds 14 in. thick or greater, which constitutes a potential resource of several billion tons, in addition to the resources reported for the coal districts discussed below.

Because the Raton Formation coarsens to the west and interfingers with beds of the overlying Poison Canyon Formation, commonly only thin coal streaks occur along the western margin of the coalfield, and thick coal deposits appear to be restricted to areas that lie east of a line that trends north to northwest about 2-3 mi west of Vermejo Park. The principal Raton Formation coal districts are defined by coal beds that lie east of Vermejo Park in the central part of the Raton coalfield. Some of these beds are shown on the Casa Grande $15^{\prime}$ geologic quadrangle map (Pillmore, 1969a). Several other districts are to the east in the adjacent Brilliant $15^{\prime}$ quadrangle, including the Brilliant district, which is partly in the Raton $15^{\prime}$ quadrangle.

\section{York Canyon District}

The York Canyon district (fig. D8) constitutes an area of about 6,500 acres east of Vermejo Park, where presently only surface mining is being carried out (the underground mine was closed in 1986). The York Canyon coal bed lies about 1,200-1,280 $\mathrm{ft}$ above the base of the Raton Formation, and it ranges in thickness from a few inches in the southeastern part of the outcrop to more than $12 \mathrm{ft}$ near the main entries of the underground mine. The coal bed is consistently at least $6 \mathrm{ft}$ thick throughout the bed area northeast of the mine entry. Measured underground coal resources in 1969 totaled 30 million tons in beds $4 \mathrm{ft}$ thick or greater (Pillmore, 1976), but the coal bed has been mined out underground where it was $5 \mathrm{ft}$ thick or greater (1988), and remaining underground resources of coal less than $5 \mathrm{ft}$ thick are uncertain. At West Ridge, on the west side of the district (fig. D8), the York Canyon coal bed and some thinner beds that overlie the York Canyon are being strip-mined. In the West Ridge deposit, remaining strippable coal reserves are estimated at about 2 million tons (Roy Pillmore, P\&M Coal Co., oral commun., 1991), and the life of the strip mine is estimated at about 3 yr. Production to date (1991) from the York Canyon coal bed totals about 20 million tons. Minable resources of strippable coal in the York Canyon district remaining after mining to date are about 2 million tons. 


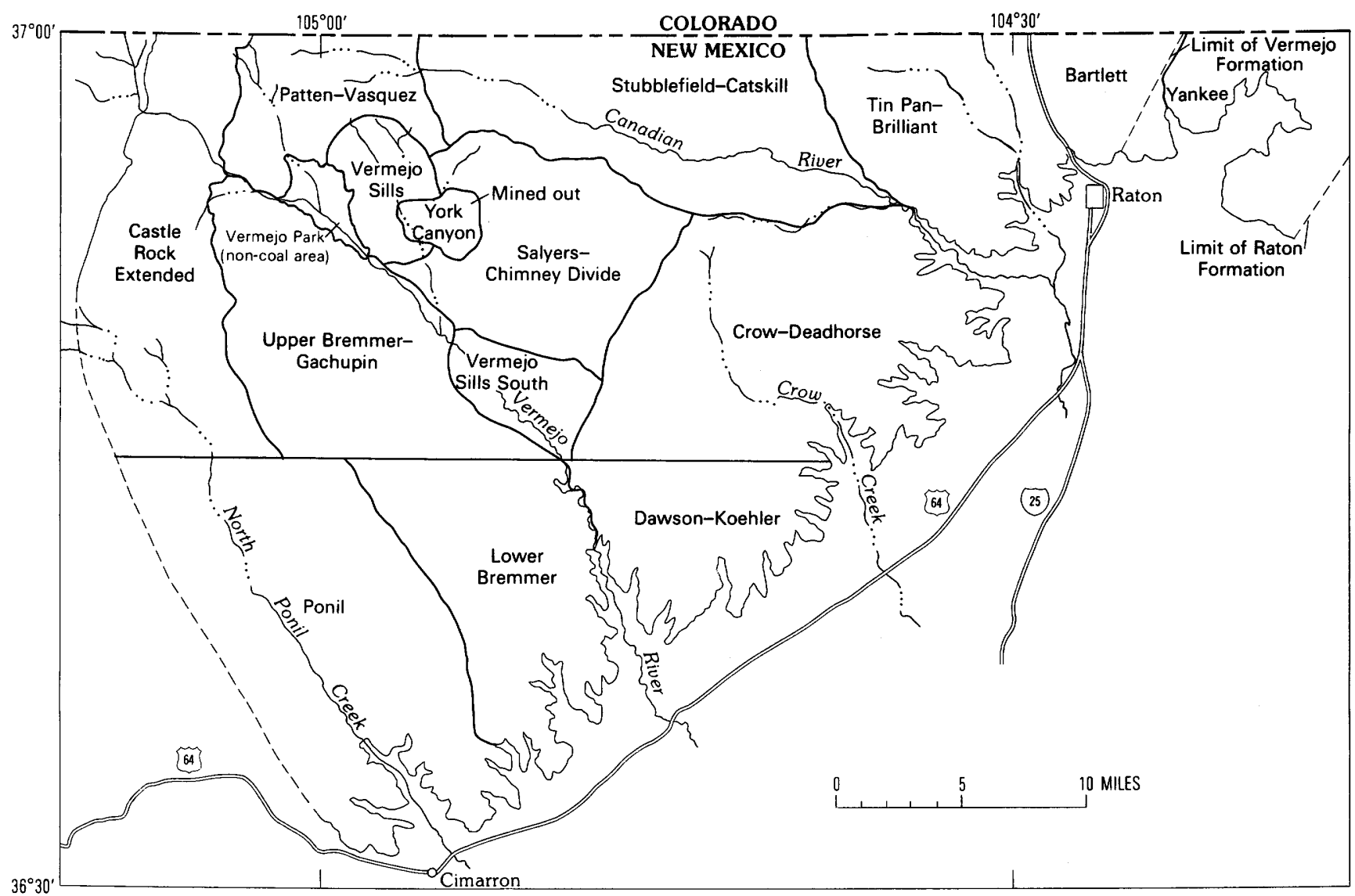

Figure D9. Geographic subdivisions of the Raton coalfield, New Mexico, used to describe inferred coal resources.

\section{Upper York (Left Fork) District}

Coal beds of the Upper York district (fig. D8) underlie nearly 9,400 acres 4 mi north of the York Canyon mine. The Left Fork coal deposit consists of two beds that are about $300 \mathrm{ft}$ beneath the York Canyon coal bed. They are about $50-60 \mathrm{ft}$ apart stratigraphically and lie $900-950 \mathrm{ft}$ above the base of the Raton Formation. These beds constitute the lowest commercial coal deposits in the Raton Formation in the central part of the coalfield. The beds range in thickness from about $31 / 2$ to $11 \mathrm{ft}$, based on extensive drilling data, and demonstrated resources exceed 130 million tons (table D4). Areas where the Left Fork coal beds possibly could be strip-mined extend north to the State line and south toward the York Canyon strip mine, but they are considered nominally economic, and the district should be regarded as a deposit to be mined primarily by underground methods. An underground test mine was opened on the upper seam in 1969, and the coal was found to be of excellent quality. Entries for a new mine called the Cimarron mine were begun late in 1983, and coal is presently being produced by longwall mining methods. Minable reserves in the upper seam presently being mined total 16 million tons, and production from the Cimarron mine will be 1.8 million tons per year (Roy Pillmore, oral commun., 1991).

\section{Cottonwood Canyon District}

The Cottonwood Canyon district (fig. D8) is about 7 mi east of York Canyon, a short distance south of the York Canyon mine road, New Mexico Highway 555. The Cottonwood Canyon coal zone is in the upper coal zone of the Raton Formation, about 1,100-1,200 ft above the base of the Raton Formation. It underlies about 4,000 acres and ranges in thickness from 3 to $8 \mathrm{ft}$. Demonstrated resources of this district are estimated at 40 million tons (table D4), 10 million of which are considered strippable, depending on market conditions and equipment used. The multiple thinseam occurrence of the coal and the dissected hill-andvalley terrain of the eastern part of the coalfield both make conventional strip-mining techniques impractical, and stripping ratios are difficult to assess. The resources considered strippable would change if demand and price of coal increased sufficiently.

\section{Ancho Canyon District}

The Ancho Canyon district (fig. D8) is considered here as two subdistricts: the Ancho, which lies north of the Vermejo River, and the Gachupin-Bracket, which lies to the south. 
Table D5. Estimated minimum inferred coal resources within subdivisions of the Raton coalfield, New Mexico

\begin{tabular}{|c|c|c|c|c|c|c|}
\hline $\begin{array}{c}\text { Subdivision or area } \\
\text { (see fig. D8) }\end{array}$ & $\begin{array}{l}\text { Area } \\
\text { (acres) }\end{array}$ & \multicolumn{2}{|c|}{ Raton Formation } & \multicolumn{2}{|c|}{ Vermejo Formation } & $\begin{array}{c}\text { Total } \\
\text { for both } \\
\text { formations } \\
\left(10^{6} \text { tons }\right)\end{array}$ \\
\hline $\begin{array}{l}\text { Bartlett } \\
\text { Yankee } \\
\text { Tin Pan-Brilliant } \\
\text { Stubblefield-Catskill } \\
\text { Patten-Vasquez }\end{array}$ & $\begin{array}{l}14,950 \\
24,310 \\
41,350 \\
59,780 \\
22,820\end{array}$ & $\begin{array}{l}6 \\
3 \\
6 \\
6 \\
5\end{array}$ & $\begin{array}{r}166 \\
134 \\
459 \\
1,106 \\
211\end{array}$ & $\begin{array}{r}2 \\
0 \\
4 \\
10 \\
4\end{array}$ & $\begin{array}{r}55 \\
0 \\
306 \\
553 \\
168\end{array}$ & $\begin{array}{r}221 \\
134 \\
765 \\
1,659 \\
379\end{array}$ \\
\hline $\begin{array}{l}\text { Castle Rock extended - } \\
\text { Upper Bremmer-Gachupin } \\
\text { Salyers-Chimney Divide } \\
\text { Crow-Deadhorse } \\
\text { York Canyon }\end{array}$ & $\begin{array}{r}53,210 \\
52,410 \\
41,350 \\
77,820 \\
3,590\end{array}$ & $\begin{array}{l}0 \\
5 \\
6 \\
4 \\
4\end{array}$ & $\begin{array}{r}0 \\
485 \\
459 \\
576 \\
26\end{array}$ & $\begin{array}{l}3 \\
4 \\
4 \\
4 \\
3\end{array}$ & $\begin{array}{r}295 \\
388 \\
306 \\
576 \\
20\end{array}$ & $\begin{array}{r}295 \\
873 \\
765 \\
1,152 \\
46\end{array}$ \\
\hline Total for all subo & divisions & s $-------n$ & 4,654 & --- & 3,474 & 8,128 \\
\hline
\end{tabular}

\footnotetext{
${ }^{1}$ Estimated average total thickness of coal.

${ }^{2}$ Modified from wanek (1963). Does not include most demonstrated resources at Dawson and Koehler districts (table D2).
}

Ancho subdistrict.-The Ancho Canyon coal zone lies roughly in the same stratigraphic position as the Cottonwood Canyon coal bed. It underlies about 2,200 acres north of the Vermejo River and contains about 25 million tons (table D4) of coal considered strippable. No underground mining is planned in this subdistrict. The coal bed lies mostly less than $100 \mathrm{ft}$ beneath the crests of ridges in this dissected portion of the coalfield. The coal occurs in a zone as much as $15 \mathrm{ft}$ thick, which contains several shale partings. These partings are expected to cause problems during mining. The maximum discrete bed thickness is about $4 \mathrm{ft}$.

Gachupin-Bracket subdistrict.-The Ancho Canyon coal zone also underlies the Gachupin-Bracket subdistrict (fig. D8). The subdistrict consists of about 1,600 acres. The zone is as thick as $9 \mathrm{ft}$ and contains coal beds as thick as 4 $\mathrm{ft}$, most of which include shale partings. Strippable demonstrated resources of the Ancho coal bed total about 9 million tons. A second coal bed, called the Bracket, lies about $40-60 \mathrm{ft}$ above the Ancho and contains estimated strippable demonstrated resources of about 2 million tons, giving a total of 11 million tons (table D4). The southwestern and western limits of the subdistrict have not been completely defined, and underground reserves may eventually be proven and outlined. No underground operations are planned at present.

\section{Chimney Divide District}

The Chimney Divide coal bed and its equivalents underlie a large area that is usually divided into two subdistricts (fig. D8):

1. The Salyers Ridge subdistrict, which includes Salyers Ridge (between Salyers and Road Canyons) and Chimney Divide (between the East Fork of Chimney Canyon and Salyers Canyon); and

2. The Caliente Canyon subdistrict, which includes the areas that lie to the east of the East Fork of Chimney Canyon, and the Caliente Canyon area. Because of the similarities of the subdistricts, they are not discussed individually.

The Chimney Divide coal bed and its equivalents crop out below the crests of the long fingerlike ridges and high drainage divides throughout an area of more than $\mathbf{5 0}$ $\mathrm{mi}^{2}$ along the drainage divide between the Vermejo and Canadian Rivers. The coal bed commonly consists of two beds about $2 \mathrm{ft}$ thick separated by a shale parting as thick as $1 \mathrm{ft}$. The maximum thickness of coal in the zone is about 4.5 ft. A coal bed termed the "L" bed locally lies 20-30 ft beneath the Chimney Divide coal bed and averages about 30 in. thick. Total demonstrated coal resources for the district exceed 60 million tons (table D4). The coal is of good 
quality for power generation but not particularly suited for metallurgical purposes. These coal beds are the highest beds in the sequence and lie near the top of the upper coal zone of the Raton Formation, about $1,800 \mathrm{ft}$ above the base.

Lee (1924) discussed the coal beds in the Raton Formation in the Brilliant and Raton quadrangles and described the thickness, stratigraphic position, and mines of each bed. Several beds as much as $8 \mathrm{ft}$ thick occur in the eastern part of the field and are of commercial value. However, most beds are less than $4 \mathrm{ft}$ thick, are areally restricted, contain partings of shale or bone, and were considered by Lee to be less desirable for the manufacture of coke. Figure D10 shows the general relations of some of the beds in the eastern part of the coalfield to those in the central part.

\section{Potato Canyon District}

The Potato Canyon coal zone underlies an area of about 6,500 acres, mainly on the ridge dividing Coal and Potato Canyons and along adjacent ridges and valleys,

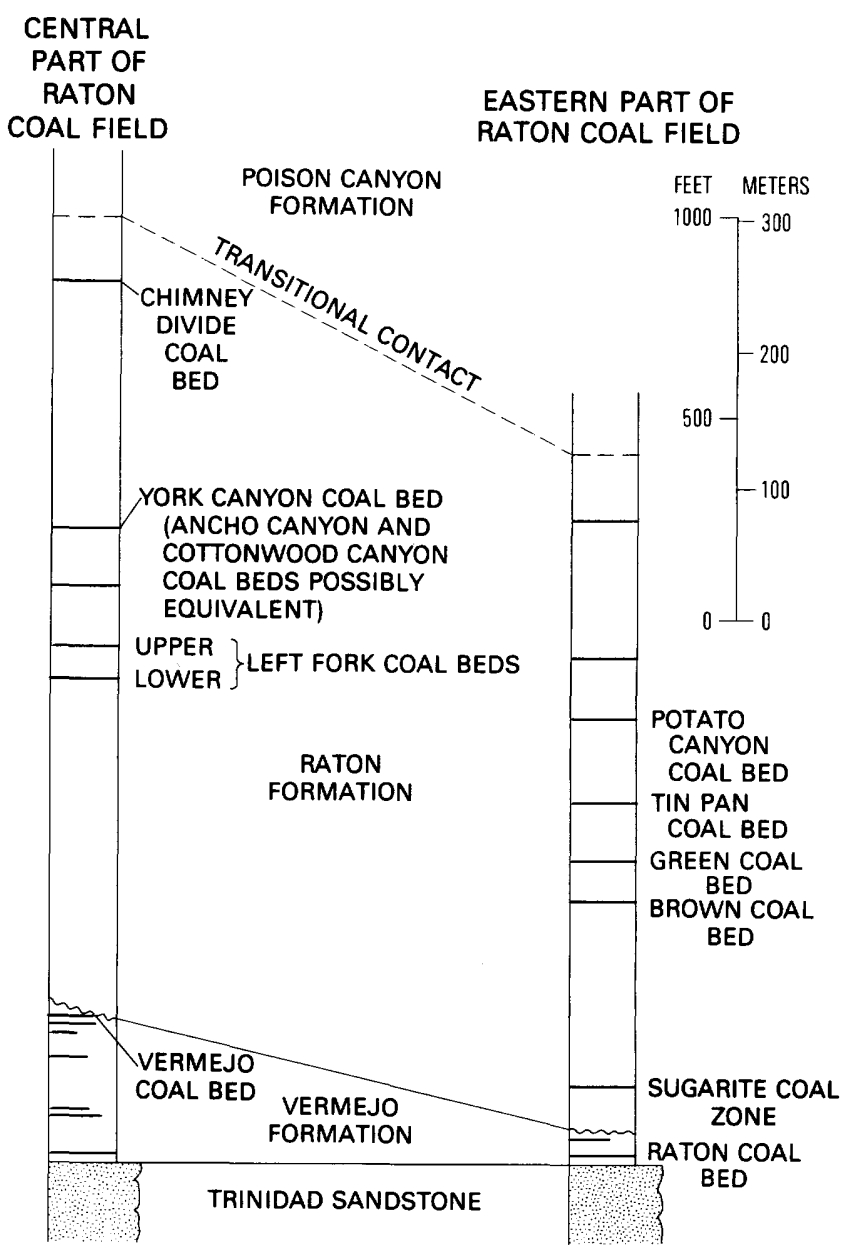

Figure D10. Diagrammatic stratigraphic section showing relations of coal beds in the central and eastern parts of the Raton coalfield, New Mexico (from Pillmore, 1976). though it also extends south into the heads of Crow and Coal Canyons. The zone lies about 950-1,050 $\mathrm{ft}$ above the Trinidad Sandstone. In Potato Canyon the zone is as thick as $8.4 \mathrm{ft}$ and has a total coal thickness as great as $7.2 \mathrm{ft}$, but individual beds within the zone rarely reach $3 \mathrm{ft}$ in thickness (Lee, 1924). The coal zone is characterized by several shale partings. It was excavated along the outcrop for several hundred yards on both sides of Potato Canyon and is also exposed in bulldozer cuts at the head of Coal Canyon. The coal zone is $6 \mathrm{ft}$ thick near the mine in Potato Canyon and is $7.5 \mathrm{ft}$ thick (including $6.3 \mathrm{ft}$ of coal) $2.5 \mathrm{mi}$ farther south, in the head of a tributary to the Canadian River called Coal Canyon. An experimental mine, opened in 1981, was driven $2,000 \mathrm{ft}$ into the south wall of Potato Canyon to test the continuity and quality of the coal. The test was completed in 1982 and the mine was closed. Total demonstrated coal resources in the district are 70.4 million tons (table D4). Small parts of the district are underlain also by the Green coal bed on the south (fig. D8) and by the Tin Pan coal bed in the northeast; both lie stratigraphically beneath the Potato Canyon coal bed.

\section{Crow Canyon District}

The Crow Canyon district (fig. D8) is defined by two areally overlapping coal beds: the Green, which is the highest stratigraphically, and the Brown. These may correlate, respectively, with the upper and lower Left Fork coal beds in the Upper York district to the west, but this speculation is based solely on stratigraphic position.

Green coal bed.-The Green coal bed crops out in the bottom of Crow Creek and has been traced in the subsurface throughout an area of several square miles. It overlies a large portion of the Brown coal bed area and extends to the south and east several miles (fig. D8). The bed occurs as a zone, as thick as $16 \mathrm{ft}$, composed of alternating thin coal layers and carbonaceous shale partings mostly less than $1 \mathrm{ft}$ thick. It lies in the lower part of the upper coal zone of the Raton Formation, approximately $700-750 \mathrm{ft}$ above the Trinidad Sandstone. The coal bed at location 386 of Lee (1924, p. 171) may be this bed; he described it as a zone $12.9 \mathrm{ft}$ thick containing $9.6 \mathrm{ft}$ of coal. Lee reported a second zone $12.5 \mathrm{ft}$ thick that lies $125 \mathrm{ft}$ stratigraphically above the Green coal bed, but it contains only $3.1 \mathrm{ft}$ of coal and is not considered economic under present conditions.

The Green coal bed underlies about the same size area as the Potato Canyon coal bed, but outcrops of the Green are sparse and thickness ranges are uncertain. The coal bed contains demonstrated coal resources of 86 million tons (table D4).

Brown coal bed.-The Brown coal bed averages $5 \mathrm{ft}$ in thickness and lies about $100 \mathrm{ft}$ beneath the Green coal bed, near the base of the upper coal zone. It is one of the lowest beds in the eastern part of the coalfield. It may correlate with the Tin Pan bed, which lies in approximately 
the same stratigraphic position in the Brilliant district. In the vicinity of Crow, Coal, and Potato Canyons, the Brown coal bed underlies a poorly defined area of several square miles, presumed to be similar in size to the area underlain by the Green coal bed, and contains demonstrated resources of 55 million tons (table D4). The Brown coal bed is about 600-650 $\mathrm{ft}$ above the Trinidad Sandstone (Kaiser Coal Corporation, written commun., 1976).

\section{Brilliant District}

Several Raton Formation coal beds occur in the Brilliant district, including the Tin Pan, Rombo Canyon, and Savage Canyon beds. These and other beds underlie ridges in the Brilliant district and parts of the Tin PanBrilliant subdivision (fig. D9). For instance, a 6-ft coal zone that crops out in Jones Canyon, a tributary of the Canadian River, about 0.7 mi west of Raton, may be equivalent to the Tin Pan coal bed or the Potato Canyon seam, but the correlation is indefinite and its lateral extent is not known. The coal resources that can be inferred to be present in these and all other coal beds in the Tin Pan-Brilliant subdivision total 459 million tons (table D5) of which 26 million tons (table D4) are the demonstrated resources in coal beds of the Raton Formation in the Brilliant district.

The Tin Pan coal bed underlies an area of about 25,000 acres, mostly north of the Canadian River, extending westward from a point about 4 mi northwest of Raton (Lee, 1924). The coal bed lies $615-720 \mathrm{ft}$ above the Trinidad Sandstone (Kaiser Coal Corporation, written commun., 1976). Near Brilliant, the zone containing the Tin Pan bed is as much as $13.3 \mathrm{ft}$ thick and has a total coal thickness of 9.4 $\mathrm{ft}$ (Lee, 1924), but individual coal beds in the zone rarely reach thicknesses greater than $5 \mathrm{ft}$ and the bed generally contains one or more shale partings. The coal bed crops out in canyons adjacent to and along Dillon Canyon and the Canadian River. Coal was produced from the Tin Pan coal bed for several years at Brilliant; but, according to Lee (1924), the coal is softer than the older coal of the Raton coal bed and produces a weak and "in some ways inferior" coke. However, a satisfactory coking blend is obtained when this coal is mixed with coal from the Raton coal bed. Separate resource estimates for the Tin Pan coal bed were not calculated because the bed was largely mined out from mines in Dillon Canyon. The unmined portions of the bed are included in resource estimates for Tin Pan-Brilliant subdivision (fig. D9; table D5).

The lowest coal bed of commercial thickness in the Raton Formation is in the Sugarite zone, which lies from 80 to more than $100 \mathrm{ft}$ above the base of the Raton Formation and extends throughout an area of about 64,000 acres in the eastern part of the coalfield (Lee, 1924). Recent subsurface investigations reveal that in the old Blossburg district of Lee (1924), west of Raton, a zone equivalent to the Sugarite is $40-50 \mathrm{ft}$ thick and lies $140-190 \mathrm{ft}$ above the base of the
Raton Formation (Kaiser Coal Corporation, written commun., 1976). The coal bed is as thick as $5.5 \mathrm{ft}$ in the mine at Sugarite and nearby localities; in other places, it is thinner and contains several shale partings. The Sugarite coal bed underlies an undetermined area beneath the basalt-capped Bartlett Mesa in the Bartlett subdivision (fig. D9), and it is also present in the northern part of the Yankee subdivision, where it underlies Barela Mesa and extends north into Colorado. Although the coal is noncoking in character, it has high heating value and was sought after as domestic fuel because its high resin content causes it to burn freely, without notable clinker (Lee, 1924).

\section{Other Coal Beds in the Raton Formation}

Parts of the Raton coalfield contain several coal beds in addition to those that define individual districts. Lee's reports $(1917 ; 1924)$ and Pillmore's reports and maps $(1969 \mathrm{a}, \mathrm{b} ; 1974 ; 1976)$ indicate that many parts of the field that have been investigated contain as much as 15 to $20 \mathrm{ft}$ of coal in beds 14 in. thick or greater. Table D5 shows minimum coal resource values based on minimum total coal thicknesses inferred in the subdivisions shown on figure D9.

Geophysical and drilling records indicate the presence of other coal beds in various areas, and field investigations have discovered several more. For instance, southwest of Vermejo Park, beds as thick as $5 \mathrm{ft}$ crop out at the heads of Pooler and Juan Baca Canyons, but these beds could not be traced along outcrop for any great distance, and subsurface data are not sufficient to define the limits of the beds. These coal beds lie at about the same stratigraphic position as those in the Upper York (Left Fork) district to the northeast. A higher coal zone, which is as thick as $9 \mathrm{ft}$, underlies about 600 acres at the head of Juan Baca Canyon, but it does not contain sufficient reserves to warrant development at this time. West of Vermejo Park, a coal bed as thick as $5 \mathrm{ft}$ underlies ridges in the drainage area of Mestas Canyon. This coal bed may also be stratigraphically equivalent to one of the Left Fork beds. Again, the bed area appears to be too small to warrant development. However, a coal zone that may have commercial importance crops out north of Vermejo Park, in Spring Canyon; it includes about 5-6 ft of coal, underlies an area of unknown extent, and warrants further investigation. The inclusion of these and other known coal beds in table D4 would significantly add to resource estimates, but detailed correlation studies would be needed to determine whether such inclusion is warranted.

Subsurface investigations in the Yankee subdivision show inferred coal resources of about 134 million tons (table D5) contained in approximately 24,000 acres, most of which lie beneath the basalt caps of Barela, Horse, and Johnson Mesas, about 3-5 mi northeast of Raton. Drilling information provided by Santa $\mathrm{Fe}$ Mining Corporation shows that about 16,000 acres of the subdivision, lying mostly beneath Barela and Horse Mesas, contains 85 
million tons of coal that could be classed as demonstrated resources. The Yankee is the easternmost subdivision in the coalfield, and the coal is contained mostly in the Sugarite coal bed (lower coal zone), and the Kellog, Dirty, and Yankee coal beds (upper coal zone). The Raton Formation thins to the east across the coalfield, and the Yankee coal bed, which is near the base of the upper coal zone, is only $300-400 \mathrm{ft}$ above the top of the Trinidad Sandstone. The Yankee coal bed is possibly stratigraphically equivalent to the Tin Pan or one of the other lower beds in the upper coal zone described above, but outcrops are obscured by soil and vegetation cover, so tracing of the beds on the ground is nearly impossible. According to Lee (1924), the coal rarely occurred in beds thicker than $2.5-3 \mathrm{ft}$, and comparatively little coal has been mined from this bed. Thicker coal occurs in the Kellog and Dirty coal beds of the district, mainly in the northern part beneath Barela Mesa. Coal beds of the upper coal zone are absent in the eastern part of the Yankee subdivision beneath the basalt flows that cap the eastern part of Johnson Mesa; it is unlikely that the Sugarite coal bed is of minable extent and thickness beneath the mesa.

Lee (1924) provides descriptions of many coal beds and zones in the eastern part of the coalfield that are not included or described here. Geophysical records of oil wells drilled in 1972, and more recent drilling by Pennzoil Corporation for coal-bed methane in 1989, suggest that coal deposits of potential commercial value underlie a large area around Van Bremmer and Cerrososo Canyons. The quality, thickness, and areal extent of any beds in this area have yet to be determined. Information from outcrops in the Cimarron area, at the southern limit of the coalfield, does not suggest the presence of thick coal beds in either the Vermejo or Raton Formation in that area.

\section{CONCLUSIONS}

The Vermejo and Raton Formations contain more than 9 billion tons of coal resources in the Raton coalfield. These resources are sufficient to sustain large-scale mining operations to meet nearly any predictable market need. However, even though the coal is of high quality and a sought-after product, difficult mining conditions and high production costs may preclude large-scale development in the face of competitively priced coal from large mines in the Powder River basin in Wyoming.

\section{REFERENCES CITED}

Baltz, E.H., 1965, Stratigraphy and history of Raton basin and notes on San Luis basin, Colorado-New Mexico: American Association of Petroleum Geologists Bulletin, v. 49, p. 2041-2075.

Ellis, R.W., 1936, Analyses of New Mexico coals: U.S. Bureau of Mines Technical Paper 569, $108 \mathrm{p}$.
Fassett, J.E., 1976, What happened during Late Cretaceous time in the Raton and San Juan basins-with some thoughts about the area in between, in Ewing, R.C., and Kues, B.S., eds., Guidebook of Vermejo Park, northeastern New Mexico: New Mexico Geological Society, 27th Field Conference, 1976, p. 185-190.

Flores, R.M., and Tur, S.M., 1982, Characteristics of deltaic deposits in the Cretaceous Pierre Shale, Trinidad Sandstone, and Vermejo Formation, Raton basin, Colorado: The Mountain Geologist, v. 19, p. 25-40.

Gill, J.R., and Cobban, W.A., 1966, The Red Bird section of the Upper Cretaceous Pierre Shale in Wyoming: U.S. Geological Survey Professional Paper 393-A, 73 p.

Lee, W.T., 1917 [1918], Geology of the Raton Mesa and other regions in Colorado and New Mexico, in Lee, W.T., and Knowlton, F.H., Geology and paleontology of Raton Mesa and other regions in Colorado and New Mexico: U.S. Geological Survey Professional Paper 101, p. 9-221.

1924, Coal resources of the Raton coal field, Colfax County, New Mexico: U.S. Geological Survey Bulletin 752, $254 \mathrm{p}$.

Pillmore, C.L., 1969a, Geologic map of the Casa Grande quadrangle, Colfax County, New Mexico, and Las Animas County, Colorado: U.S. Geological Survey Geologic Quadrangle Map GQ-823, scale 1:62,500.

$1969 \mathrm{~b}$, Geology and coal deposits of the Raton coal field, Colfax County, New Mexico: Mountain Geologist, v. 6, p. 125-142.

1974, Geologic and structure contour maps of the Ute Creek $1^{\circ}$ SE quadrangle, Colfax County, New Mexico: U.S. Geological Survey Open-File Report 74-1086, 2 sheets, scale $1: 24,000$.

1976, Commercial coal beds of the Raton coal field, Colfax County, New Mexico, in Ewing, R.C., and Kues, B.S., eds., Guidebook of Vermejo Park, northeastern New Mexico: New Mexico Geological Society, 27th Field Conference, 1976, p. 227-247.

Pillmore, C.L. and Flores, R.M., 1984, Field guide and discussions of coal deposits, depositional environments, and the Cretaceous-Tertiary boundary, southern Raton basin, New Mexico and Colorado, in Lintz, Joseph, Jr., ed., Western geological excursions, v. 3: Geological Society of America Guidebook, Annual Meeting, Reno, Nev., 1984; Reno, Nev., MacKay School of Mines, Department of Geology, p. 1-55.

1987, Stratigraphy and depositional environments of the Cretaceous-Tertiary boundary interval and associated rocks, Raton basin, New Mexico and Colorado, in Fassett, J.E., and Rigby, J.K., Jr., eds., The Cretaceous-Tertiary boundary in the San Juan and Raton basins, New Mexico and Colorado: Geological Society of America Special Paper 209, p. 111-130.

Pillmore, C.L., Tschudy, R.H., Orth, C.J., Gilmore, J.S., and Knight, J.D., 1984, Geologic framework of the Ir anomaly at selected $\mathrm{K} / \mathrm{T}$ boundary sites, Raton basin, New Mexico and Colorado: Science, v. 223, no. 4641, p. 1180-1182.

Read, C.B., Duffner, R.T., Wood, G.H., Jr., and Zapp, A.D., 1950, Coal resources of New Mexico: U.S. Geological Survey Circular 89, 24 p. 
Scott, G.R., and Pillmore, C.L., 1989, Geologic and structure contour maps of the Raton $1 / 2^{\circ} \times 1^{\circ}$ quadrangle, Colfax and Union Counties, New Mexico and Las Animas County, Colorado: U.S. Geological Survey Open File Report 89-282, 2 sheets, scale 1:100,000.

Scott, G.R., Wilcox, R.E., and Mehnert, H.H., 1990, Geology of volcanic and subvolcanic rocks of the Raton-Springer area, Colfax county, New Mexico: U.S. Geological Survey Professional Paper 1507, 58 p.

St. John, Orestes, 1896, Report on the Vermejo Northside coal area [letter to Maxwell Land Grant Company], reproduced in Pillmore, C.L., 1976, Third day road $\log * * *$, in Ewing,
R.C., and Kues, B.S., eds., Guidebook of Vermejo Park, northeastern New Mexico: New Mexico Geological Society, 27th Field Conference, 1976, p. 66-68.

Trumbull, J.V.A., 1959, Coal fields of the United States: U.S. Geological Survey Map, scale 1:5,000,000.

Wanek, A.A., 1963, Geology and fuel resources of the southwestern part of the Raton coal field, Colfax County, New Mexico: U.S. Geological Survey Coal Investigations Map C-45, 2 sheets, scale 1:48,000.

Wood, G.H., Jr., Kehn, T.M., Carter, M.D., and Culbertson, W.C., 1983, Coal resource classification system of the U.S. Geological Survey: U.S. Geological Survey Circular 891, $65 \mathrm{p}$. 
Chapter E

\section{Geology and Coal Resources of New Mexico's Small Coalfields}

BY FRANK W. CAMPBELL, GRETCHEN K. HOFFMAN, FRANK E. KOTTLOWSKI, and BRIAN W. ARKELL New Mexico Bureau of Mines and Mineral Resources

Prepared in cooperation with the New Mexico Bureau of Mines and Mineral Resources

Descriptions of 11 fields outside the San Juan and Raton basins, which may hold more than 2 billion tons of coal

U.S. GEOLOGICAL SURVEY BULLETIN 1972

COALFIELDS OF NEW MEXICO: GEOLOGY AND RESOURCES 


\title{
CONTENTS
}

\author{
Abstract 71 \\ Introduction 71 \\ Salt Lake field $\mathbf{7 1}$ \\ Datil Mountains field $\mathbf{7 3}$ \\ Sierra Blanca field $\mathbf{7 3}$ \\ Rio Puerco field $\mathbf{7 4}$ \\ Tierra Amarilla field $\mathbf{7 4}$ \\ Cerrillos field $\mathbf{7 5}$ \\ Carthage field $\mathbf{7 5}$ \\ Hagan field $\mathbf{7 5}$ \\ Tijeras field $\quad \mathbf{7 6}$ \\ Jornada del Muerto field $\mathbf{7 6}$ \\ Engle field $\mathbf{7 6}$ \\ References cited $\quad \mathbf{7 6}$ \\ FIGURE
}

E1. Map showing coalfields, rail lines, and pertinent localities in New Mexico 


\title{
Geology and Coal Resources of New Mexico's Small Coalfields
}

\author{
By Frank W. Campbell, Gretchen K. Hoffman, Frank E. Kottlowski, and \\ Brian W. Arkell \\ New Mexico Bureau of Mines and Mineral Resources
}

\begin{abstract}
The small coalfields of New Mexico, outside the San Juan basin and the Raton field, may contain resources of more than 2 billion tons of coal, which is mostly subbituminous but includes some anthracite and coking coals. Fields with reasonable economic reserves are the Salt Lake, Sierra Blanca, Datil Mountains, Cerrillos, and Carthage fields. The coal beds are in the lower part of the Upper Cretaceous Mesaverde Group or its equivalents, and the coals have medium ash content (10-20 percent) and low sulfur content (less than 1 percent).
\end{abstract}

\section{INTRODUCTION}

Most of New Mexico's coal is in two major areas: the San Juan basin in the northwest in the Colorado Plateau physiographic province, and the Raton area in the northcentral part of the State in the Great Plains province. The other coalfields in the State are relatively small and are in the southeastern part of the Basin and Range province and the southeastern part of the Colorado Plateau province.

Among these "small" coalfields (Tabet and Frost, 1978), the ones that contain the largest amounts of resources are the Salt Lake field and the Datil Mountains field in west-central New Mexico and the Sierra Blanca field in south-central New Mexico (fig. E1). The Cerrillos and Carthage fields have been mined extensively in the past and still contain usable resources. The Hagan, Tijeras, Jornada del Muerto, and Engle coalfields contain lesser amounts of coal resources. The Rio Puerco and Tierra Amarilla fields are outliers of the San Juan basin coal sequences.

These small coalfields are of considerable historical interest. The Spanish used small amounts of coal from

Manuscript approved for publication January 10, 1991. several of them as early as the 17th century. Anthracite was mined from the Cerrillos field as early as 1835. Mining began on a significant scale in 1861, when the U.S. Army troops at Fort Craig opened a mine in the Carthage field. Present economic interest in the small coalfields is largely confined to the Sierra Blanca field, because of its location on the Southern Pacific Railway, and the Salt Lake coalfield, which lies about 50 miles east of the power plants in east-central Arizona.

\section{SALT LAKE FIELD}

The Salt Lake coalfield (fig. E1), in west-central New Mexico, covers about $750 \mathrm{mi}^{2}$ straddling the Cibola-Catron County line in Tps. 1-8 N. and Rs. 15-21 W. This is a transitional area between the Zuni-Gallup basin to the north and the Datil-Mogollon volcanic field to the south. New Mexico Highway 32 provides access to U.S. Highway 60 to the south at Quemado and northward to Interstate 40 at Gallup.

Structurally, the Salt Lake field is simple, with a few low-displacement normal faults, and some small-scale flexures. Dips are generally less than $5^{\circ}$.

Upper Cretaceous coal-bearing units crop out in a westward-opening arcuate belt along Carrizo Creek. Most of the coal beds occur in the Moreno Hill Formation (McLellan and others, 1983a), which correlates northward and eastward with the Carthage and Fite Ranch Members of the Tres Hermanos Formation, the Pescado Tongue of the Mancos Shale, the Gallup Sandstone, and the lower part of the Crevasse Canyon Formation (Hook and others, 1983).

Minable coals are in the lower member of the Moreno Hill Formation, which has a maximum thickness of $798 \mathrm{ft}$; individual beds reach a maximum thickness of $13.2 \mathrm{ft}$ but average $4.1 \mathrm{ft}$. Some thin coals $(1-3 \mathrm{ft})$ are found in the Upper Cretaceous Dakota Sandstone in the western part of 


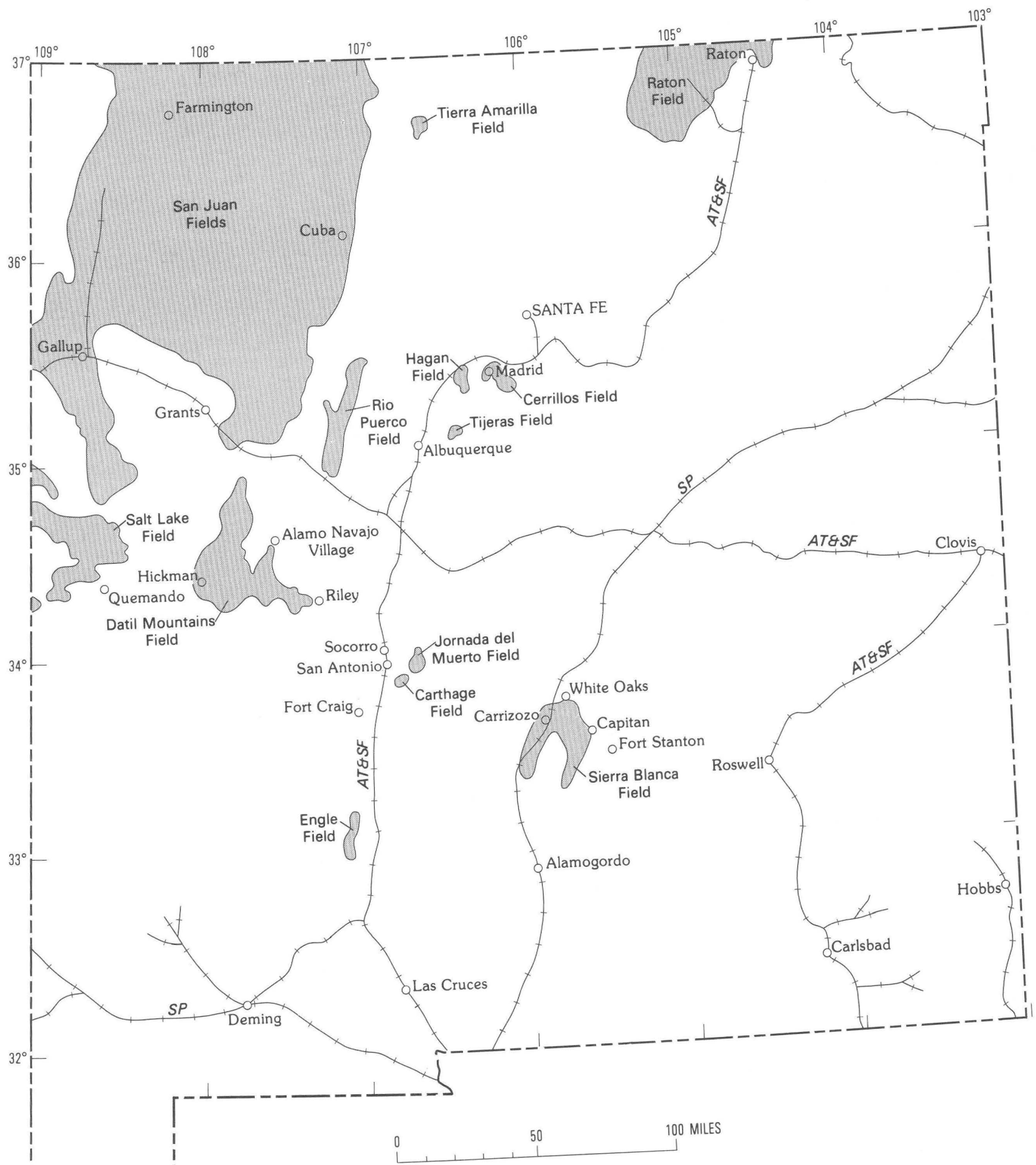

L__ _ _ _ _ Railroads identified here are the Atchison, Topeka, Figure E1. Coalfields, rail lines, and pertinent localities
and Santa Fe (AT\&SF) and the Southern Pacific (SP). 
the field. Coals in the Salt Lake field are of a high-volatile $\mathrm{C}$ to $\mathrm{B}$ bituminous rank, as shown below:

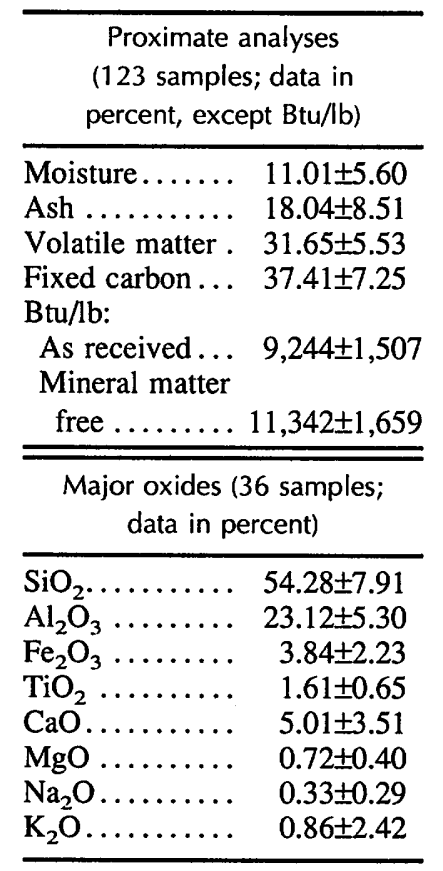

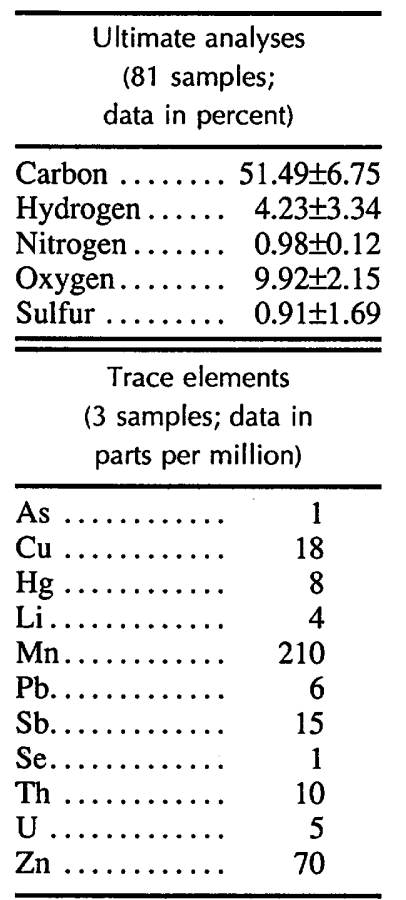

The Salt Lake coalfield is estimated to have coal resources of 480 million tons (coal beds with minimum thickness of $1.2 \mathrm{ft}$ ) and coal reserves of 240 million tons (minimum thickness of $2.5 \mathrm{ft}$ ), based on recent geologic mapping, drilling, and coal-resource evaluation of the field by the New Mexico Bureau of Mines and Mineral Resources and the U.S. Geological Survey (Anderson, 1981, 1982; Campbell, 1981, 1984; McLellan, Haschke, and Robinson, 1982; McLellan, Robinson, and others, 1982; McLellan, Haschke, and others, 1983a, b; McLellan, Robinson, and Haschke, 1983; McLellan and others, 1984; Roybal, 1982; and Roybal and Campbell, 1981). Out of these total resources and reserves, 144 million tons has a stripping ratio of 20:1 or less (Campbell and Roybal, 1984).

\section{DATIL MOUNTAINS FIELD}

The Datil Mountains coalfield (fig. E1) lies at the junction of Socorro, Catron, and Cibola Counties, in the northern foothills of the Datil, Gallinas, and Bear Mountains, generally in the upper drainage basin of the Rio Salado between Riley and Hickman. The field covers about $760 \mathrm{mi}^{2}$. Access to the rugged area is by ranch roads and New Mexico Highway 52 to Alamo.

The geological structure of the field is complicated by broad-scale folding, igneous intrusions, and faulting, mostly of Laramide and Tertiary age. Southward the coal-bearing units are overlain by thick volcanic tuffs and flows. The coal beds are in the Tres Hermanos and Crevasse Canyon Formations. The Crevasse Canyon Formation coal beds are the time equivalent of the Dilco Coal Member of the Crevasse Canyon Formation in the San Juan and Acoma basins (Molenaar, 1974). Coals range from subbituminous A to high-volatile $\mathrm{C}$ bituminous in rank, as shown below:

\begin{tabular}{|c|c|}
\hline \multicolumn{2}{|c|}{$\begin{array}{c}\text { Proximate analyses } \\
\text { (26 samples; data in } \\
\text { percent, except Btu/lb) }\end{array}$} \\
\hline $\begin{array}{l}\text { Moisture...... } \\
\text { Ash .......... } \\
\text { Volatile matter } \\
\text { Fixed carbon ... } \\
\text { Btu/lb: } \\
\text { As received... } \\
\text { Mineral matter } \\
\text { free ......... }\end{array}$ & $\begin{array}{r}7.42 \pm 5.66 \\
12.50 \pm 5.60 \\
36.20 \pm 4.00 \\
43.77 \pm 4.63 \\
10,871 \pm 1,597 \\
12,453 \pm 1,712\end{array}$ \\
\hline \multicolumn{2}{|c|}{$\begin{array}{l}\text { Major oxides ( } 7 \text { samples; } \\
\text { data in percent) }\end{array}$} \\
\hline 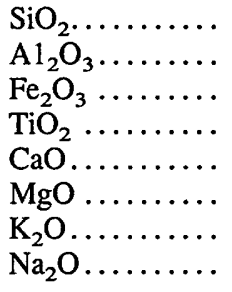 & $\begin{array}{r}58.42 \pm 8.22 \\
16.83 \pm 1.85 \\
4.64 \pm 0.72 \\
1.22 \pm 0.17 \\
4.59 \pm 0.86 \\
1.35 \pm 0.41 \\
0.81 \pm 0.19 \\
0.50 \pm 0.18\end{array}$ \\
\hline
\end{tabular}

Ultimate analyses
(26 samples;
data in percent)

Carbon ....... 62.41 \pm 6.77

Hydrogen ..... 4.98 40.42

Nitrogen ....... $1.10 \pm 0.19$

Oxygen....... $0.46 \pm 8.37$

Sulfur ....... $0.57 \pm 0.12$

Trace elements

(3 samples; data in parts per million)

\begin{tabular}{|c|c|}
\hline As $\ldots . . . \ldots \ldots$ & $8 \pm 9$ \\
\hline $\mathrm{Cu} \ldots \ldots \ldots \ldots$ & $15 \pm 11$ \\
\hline $\mathrm{Hg} \ldots \ldots \ldots \ldots$ & $0.35 \pm 0.31$ \\
\hline $\mathrm{Li} \ldots \ldots \ldots \ldots$. & $11 \pm 7$ \\
\hline Mn............ & $151 \pm 330$ \\
\hline $\mathrm{Ni} . \ldots \ldots \ldots \ldots$ & $8 \pm 4$ \\
\hline $\mathrm{Pb} . \ldots \ldots \ldots \ldots$ & $8 \pm 4$ \\
\hline Sb.............. & $13 \pm 17$ \\
\hline Se............ & $3 \pm 2$ \\
\hline Th $\ldots \ldots \ldots \ldots$ & $8 \pm 4$ \\
\hline $\mathrm{U} \ldots \ldots \ldots \ldots$ & $3 \pm 1$ \\
\hline $\mathrm{Zn} \ldots \ldots \ldots \ldots$ & $14 \pm 9$ \\
\hline
\end{tabular}

Read and others (1950) estimated resources of the Datil Mountains area as more than a billion tons, but recent detailed mapping and drilling suggest this estimate is too high. For the eastern part of the field, Osburn $(1982,1983$, 1984) estimated measured and indicated resources as 85 million tons in beds averaging $2.7 \mathrm{ft}$ in thickness and attaining a maximum of $4 \mathrm{ft}$. However, Winchester (1921) reported thicker coal beds in the northwest part of the coalfield.

\section{SIERRA BLANCA FIELD}

The Sierra Blanca field, also known as the Capitan field, is in south-central New Mexico, on the northeast margin of the Tularosa basin, in Lincoln and Otero Counties. Coal-bearing units crop out on the edges of the Sierra Blanca syncline and also flank the extrusive-intrusive complex of Sierra Blanca on its east, north, and west sides, covering about $435 \mathrm{mi}^{2}$. Structurally, the field is a northplunging syncline, which has limbs dipping $15^{\circ}-29^{\circ}$. The geology of the field is further complicated by many faults, dikes, and sills.

U.S. Highway 54 and the Southern Pacific Railway cut across the west edge of the field, and U.S. Highway 380 trends southeastward across the northern part. The coal seams have been mined near Capitan, White Oaks, Fort Stanton, and Carrizozo. 
Minable coals appear to be southeastward equivalents of the Crevasse Canyon Formation (Arkell, 1983; Hook and others, 1983). Available analyses indicate that the coals are high-volatile $\mathrm{C}$ and $\mathrm{B}$ bituminous:

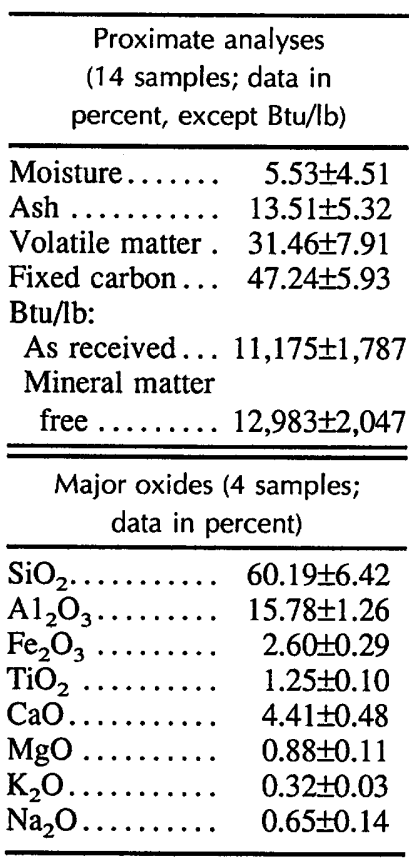

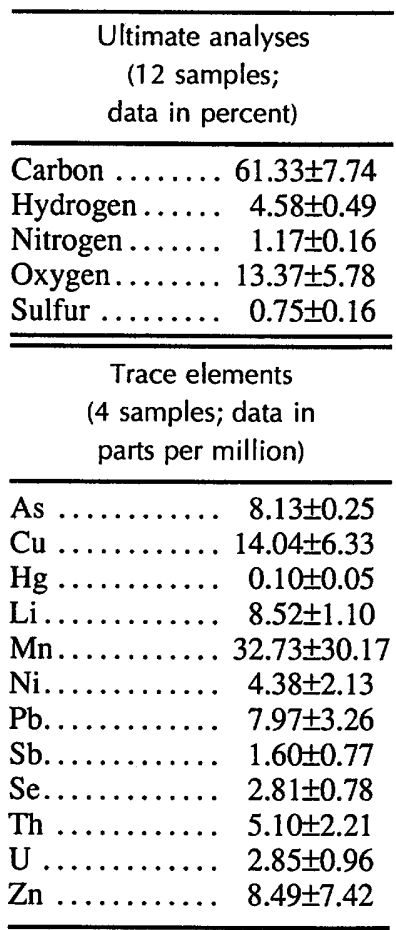

Where they are near igneous intrusions, the coals are of coking quality. Read and others (1950) estimated original resources of about 1.6 billion tons. However, the steep dips of many of the coal beds and the numerous faults, dikes, and sills, make mining difficult. Coal beds north of Carrizozo may be favorable for strip mining, but reserve calculations for them are proprietary. Seam thickness, as determined from sparse data, average $2.3 \mathrm{ft}$, but several lenses near Capitan (Bodine, 1956) are as much as $7 \mathrm{ft}$.

\section{RIO PUERCO FIELD}

The Rio Puerco coalfield is a small outlier of the San Juan basin (fig. E1), lying about 15 miles southeast of the main basin. Interstate 40 crosses the southern tip of the field, along the lower part of Rio Puerco drainage. appear to range from subbituminous $\mathrm{A}$ to high-volatile $\mathrm{C}$ bituminous in rank, based on analyses of three as-received samples:

\begin{tabular}{lr}
\hline \multicolumn{2}{c}{$\begin{array}{c}\text { Proximate analyses } \\
\text { (3 samples; data in } \\
\text { percent, except Btu/lb) }\end{array}$} \\
\hline Moisture...... & $16.27 \pm 5.84$ \\
Ash ......... & $8.60 \pm 1.64$ \\
Volatile matter . & $34.67 \pm 2.62$ \\
Fixed Carbon ... & $40.47 \pm 1.82$ \\
Btu/lb: & \\
As received ... & $9,473 \pm 195$ \\
Mineral matter & \\
free ......... & $10,452 \pm 208$ \\
\hline
\end{tabular}

\begin{tabular}{cr}
\hline $\begin{array}{c}\text { Ultimate analyses } \\
\text { (2 samples; } \\
\text { data in percent) }\end{array}$ \\
\hline Carbon ........ & $55.05 \pm 0.85$ \\
Hydrogen ..... & $6.15 \pm 0.35$ \\
Nitrogen ...... & $1.00 \pm 0.00$ \\
Oxygen ........ & $29.00 \pm 1.60$ \\
Sulfur ........ & $0.93 \pm 0.41$ \\
\hline
\end{tabular}

All data on bed characteristics and coal quality for the Rio Puerco field are based on outcrop data, as no drilling data are available. The total reserves for the northern part of this field, within $250 \mathrm{ft}$ of the surface, are estimated at 185 million tons. Resources $(0-250 \mathrm{ft})$ for the southern part of the Rio Puerco field are estimated at 175 million tons. Because of the paucity of data for these coal beds, the steep dip of the beds, and the abundance of high-angle faults within them, the coal resource potential in this field is considered to be low. Information that could be gained by drilling would help to clarify the true coal potential and the extent of the coal-bearing formation.

\section{TIERRA AMARILLA FIELD}

The Tierra Amarilla coalfield in north-central New Mexico, in the southeast corner of the Tierra Amarilla Land Grant and in the eastern part of the Chama basin, is an outlier of the San Juan basin, about 12 miles east of the eastern margin. U.S. Highway 84 skirts the east edge of the field.

The coal beds, in the Menefee Formation of the Mesaverde Group, are thin and lenticular; the maximum coal seam thickness is $4.1 \mathrm{ft}$. Two analyses indicate the coals are subbituminous $\mathrm{A}$ in rank and give these averages:
The coal-bearing units are within the Rio Puerco fault zone occur in narrow, steeply dipping fault blocks outlined by a north-northeast-trending swarm of high-angle faults on the border between the Rio Grande rift and the San Juan basin.

Outcropping coal beds are in the Dilco and Gibson Coal Members of the Crevasse Canyon Formation. The Dilco coal beds are thin; those in the Gibson Member appear to average $2.5 \mathrm{ft}$ in thickness, although seams as thick as $9.6 \mathrm{ft}$ have been mined for local use in the northern part of the field (Shomaker and others, 1971). The coal beds

\begin{tabular}{lr}
\hline \multicolumn{2}{c}{$\begin{array}{l}\text { Proximate analyses } \\
\text { (2 samples; data in }\end{array}$} \\
percent, except Btu/lb) \\
\hline Moisture....... & $18.70 \pm 0.80$ \\
Ash .......... & $8.50 \pm 0.80$ \\
Volatile matter . & $32.60 \pm 0.50$ \\
Fixed Carbon .. & $40.20 \pm 1.10$ \\
Btu/lb: \\
As received ... & $9,875 \pm 235$ \\
Mineral matter \\
free ........
\end{tabular}

\begin{tabular}{|c|c|}
\hline \multicolumn{2}{|c|}{$\begin{array}{l}\text { Ultimate analyses } \\
\qquad \text { ( } 1 \text { sample; } \\
\text { data in percent) }\end{array}$} \\
\hline Carbon ......... & $57.20 \pm 0.00$ \\
\hline Hydrogen ...... & $6.10 \pm 0.00$ \\
\hline Nitrogen ....... & $1.30 \pm 0.00$ \\
\hline Oxygen......... & $26.70 \pm 0.00$ \\
\hline Sulfur ........ & $1.05 \pm 0.05$ \\
\hline
\end{tabular}


Landis and Dane (1969) estimated the total resources to be 4.5 million tons of inferred coal in the Tierra Amarilla field.

\section{CERRILLOS FIELD}

The Cerrillos field is in north-central New Mexico, in the broken foothill country north of the Ortiz Mountains and south of the broad valley of Rio Galisteo; it is traversed by the main line of the Atchison, Topeka, and Santa Fe Railway. New Mexico Highway 14 crosses the field from south to north and serves the main town, Madrid.

The field is a complex syncline in which the coalbearing rocks have been broken by many faults and have been intruded by swarms of dikes and sills. Near the thick igneous intrusions the coals have been metamorphosed to semianthracite and anthracite.

The coal-bearing strata are in the Mesaverde Formation. The major beds are as much as $6 \mathrm{ft}$ in thickness and have yielded considerable tonnages of anthracite and bituminous coals. Some of the bituminous lenses are of medium coking coal. Anthracite is restricted to the parts of the White Ash seam that are near the intrusive rocks and to a thinner, unnamed seam immediately overlying the intrusive body.

As much as 45,000 tons of anthracite was mined annually from the Cerrillos field during the period 1888 to 1957 and was supplied to users throughout the central and western parts of the nation. Read and others (1950) estimated resources of 47.5 million tons of bituminous coal and 5.7 million tons of anthracite. Analyses of 82 samples as-received gave the following averages:

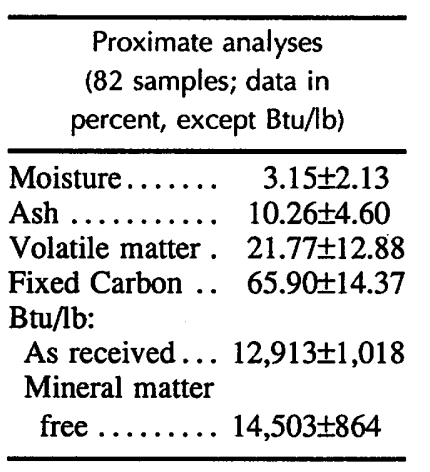

\section{CARTHAGE FIELD}

The Carthage coalfield lies in central New Mexico on the northwest edge of Jornada del Muerto, an extensive syncline-graben of that semiarid region. U.S. Highway 380 cuts across the northern edge of the field. The area is 8 miles east of San Antonio, which is served by the Atchison, Topeka, and Santa Fe Railway and by Interstate Highway 25.
Two coal seams, ranging from 4 to $7 \mathrm{ft}$ in thickness, occur in the Crevasse Canyon Formation. Only the lower coal, the Carthage seam, has been mined; it is excellent coking coal of high-volatile $\mathrm{C}$ bituminous rank.

This small field occupies about $10 \mathrm{mi}^{2}$ and is cut into a mosaic of small fault blocks, making mining difficult and expensive. U.S. Army troops stationed at nearby Fort Craig opened mining in this field in 1861, the earliest "largescale" mining in New Mexico. In the late 1800's and early 1900 's, the Carthage field supplied coking coal to many smelters in southwestern New Mexico and northern Mexico, but between 1950 and 1975 only a single small underground mine operated intermittently to provide coal for local heating (Kottlowski and others, 1984).

Most of the easily mined coal has been removed; remaining reserves may be as much as $\mathbf{3 0}$ million tons. The averages of 11 analyses on an as-received basis follow:

\begin{tabular}{lr}
\hline \multicolumn{2}{c}{$\begin{array}{c}\text { Proximate analyses } \\
\text { (11 samples; data in } \\
\text { percent, except Btu/lb) }\end{array}$} \\
\hline Moisture....... & $3.36 \pm 1.30$ \\
Ash ......... & $11.55 \pm 3.01$ \\
Volatile matter. & $36.55 \pm 2.64$ \\
Fixed carbon... & $48.68 \pm 3.08$ \\
Btu/lb: \\
As received ... \\
Mineral matter \\
free ......... \\
\hline
\end{tabular}

\begin{tabular}{cr}
\hline $\begin{array}{c}\text { Ultimate analyses } \\
\text { (4 samples; } \\
\text { data in percent) }\end{array}$ \\
\hline Carbon ....... & $69.38 \pm 3.52$ \\
Hydrogen ...... $5.25 \pm 0.21$ \\
Nitrogen ...... & $1.28 \pm 0.11$ \\
Oxygen....... & $12.50 \pm 3.66$ \\
Sulfur ........ & $0.83 \pm 0.15$
\end{tabular}

\section{HAGAN FIELD}

The Hagan field lies in north-central New Mexico in a dissected valley drained by Tongue Arroyo northeast of the Sandia Mountains and southwest of Ortiz Mountain.

The coal seams are 3-5 ft thick, occur in the Mesaverde Group, are of high-volatile $\mathrm{C}$ bituminous rank, and are cut by numerous faults. Several small underground mines operated near Hagan in the early 1900's and again from 1927 to 1939 (Kelley and Northrop, 1975). The field's remoteness and difficulties of mining caused by the complex geologic structure closed the mining operations. Read and others (1950) estimated resources of 17.3 million tons for this field (then called the Una del Gato field). The averages of 10 analyses on an as-received basis follow:

\begin{tabular}{lr}
\hline \multicolumn{2}{c}{$\begin{array}{c}\text { Proximate analyses } \\
\text { (10 samples; data in } \\
\text { percent, except Btu/lb) }\end{array}$} \\
\hline Moisture...... & $10.56 \pm 2.14$ \\
Ash ......... & $8.26 \pm 2.42$ \\
Volatile matter. & $38.00 \pm 3.90$ \\
Fixed carbon ... & $43.20 \pm 3.09$ \\
Btu/lb: & \\
As received ... & $10,508 \pm 1,477$ \\
Mineral matter & \\
free ......... & $11,625 \pm 1,653$ \\
\hline
\end{tabular}

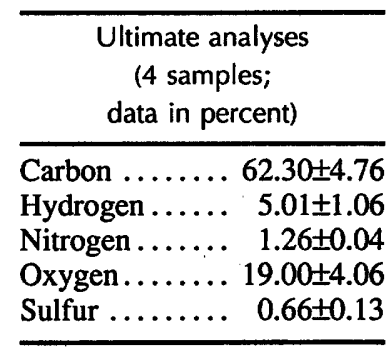

New Mexico's Small Coalfields 


\section{TIJERAS FIELD}

The Tijeras field is amid the rolling eastern foothills of the Sandia Mountains in north-central New Mexico. The coal-bearing rocks are in the Mesaverde Group and occur in a small down-dropped fault block, the Tijeras graben. The strata are folded into two synclines and an intervening anticline, and they dip steeply (Kelley and Northrop, 1975). Several thin beds of bituminous rank coal crop out, but only a few tons have been mined for minor local use. Read and others (1950) estimated resources of 1.6 million tons. The averages of three as-received analyses follow (proximate analyses; results in percent, except Btu/lb):

\begin{tabular}{ll}
\hline Moisture $\ldots \ldots \ldots \ldots \ldots \ldots \ldots$ & $4.20 \pm 3.82$ \\
Ash $\ldots \ldots \ldots \ldots \ldots \ldots \ldots$ & $15.83 \pm 10.81$ \\
Volatile matter $\ldots \ldots \ldots \ldots \ldots$ & $34.93 \pm 2.76$ \\
Fixed carbon $\ldots \ldots \ldots \ldots \ldots$ & $45.03 \pm 7.11$ \\
Btu/lb: & \\
As received $\ldots \ldots \ldots \ldots \ldots$ & $11,104 \pm 1,997$ \\
Mineral matter free ........ & $13,631 \pm 2,424$
\end{tabular}

\section{JORNADA DEL MUERTO FIELD}

Five to twenty miles east and northeast of the Carthage field, Mesaverde Group coal-bearing rocks underlie the northwest edge of Jornada del Muerto. Windblown sand conceals much of the bedrock, the area is remote, and there has been no appreciable mining. The few known outcrops of coal have maximum thickness of only 3 $\mathrm{ft}$ (Tabet, 1979a). However, drilling might delineate additional reserves, as the Cretaceous strata extend for at least 10 miles in a north-south direction. Analyses of three coal samples as received show an average composition similar to that of the Carthage field coals (proximate analyses; results in percent, except Btu/lb):

\begin{tabular}{lc}
\hline Moisture $\ldots \ldots \ldots \ldots \ldots \ldots \ldots$ & $2.3 \pm .0 .64$ \\
Ash $\ldots \ldots \ldots \ldots \ldots \ldots \ldots \ldots$ & $16.3 \pm 5.80$ \\
Volatile matter $\ldots \ldots \ldots \ldots \ldots$ & $38.2 \pm 3.01$ \\
Fixed carbon $\ldots \ldots \ldots \ldots \ldots \ldots$ & $43.1 \pm 4.71$ \\
Sulfur $\ldots \ldots \ldots \ldots \ldots \ldots \ldots$ & $2.3 \pm 0.64$ \\
Btu/lb: & \\
As received $\ldots \ldots \ldots \ldots \ldots \ldots$ & $11,410 \pm 1,353$ \\
Mineral matter free $\ldots \ldots \ldots$ & $13,897 \pm 700$ \\
\hline
\end{tabular}

\section{ENGLE FIELD}

The geologic setting of the Engle field is similar to that of the Jornada del Muerto field, although the Engle field is about 50 miles farther south. It lies on the west edge of the Jornada del Muerto syncline on the alluvial fans east of the Caballo Mountains. Prospect pits have opened thin lenses of coal, and drill holes have penetrated several coal beds in the Mesaverde Group, but the apparent maximum thickness of coal seams is $4 \mathrm{ft}$. Information from drilling indicates 32 million tons of deep coal (Tabet, 1979b) having an average as-received composition as follows (proximate analyses; results in percent, except Btu/lb):

\begin{tabular}{cc}
\hline Moisture $\ldots \ldots \ldots \ldots \ldots \ldots \ldots$ & $6.5 \pm 5.50$ \\
Ash $\ldots \ldots \ldots \ldots \ldots \ldots \ldots$ & $20.0 \pm 0.15$ \\
Volatile matter $\ldots \ldots \ldots \ldots \ldots$ & $18.9 \pm 6.41$ \\
Fixed carbon $\ldots \ldots \ldots \ldots \ldots \ldots$ & $54.7 \pm 12.30$ \\
Sulfur $\ldots \ldots \ldots \ldots \ldots \ldots \ldots$ & $0.5 \pm 0.12$ \\
Btu/lb: & \\
As received $\ldots \ldots \ldots \ldots \ldots$ & $9,745 \pm 2,080$ \\
Mineral matter free $\ldots \ldots \ldots$ & $12,429 \pm 2,637$ \\
\hline
\end{tabular}

\section{REFERENCES CITED}

Anderson, O.J., 1981, Geologic map of the Cantaralo Spring quadrangle, Cibola County, New Mexico: New Mexico Bureau of Mines and Mineral Resources Open-File Report 142.

1982, Geology and coal resources of the Venadito Camp quadrangle, Cibola County, New Mexico: New Mexico Bureau of Mines and Mineral Resources Open-File Report 163.

Arkell, B.W., 1983, Geology and coal resources of the Cub Mountain area, Sierra Blanca coal field, New Mexico: Socorro, N. Mex., New Mexico Institute of Mining and Technology, M.S. thesis, 104 p.

Bodine, M.W., Jr., 1956, Geology of Capitan coal field, Lincoln County, New Mexico: New Mexico Bureau of Mines and Mineral Resources Circular 35, 19 p.

Campbell, F.W., 1981, Geology and coal resources of Cerro Prieto and The Dyke quadrangles: New Mexico Bureau of Mines and Mineral Resources Open-File Report 144, 44 p.

1984, Geology and coal resources of the Cerro Prieto and The Dyke quadrangles: New Mexico Bureau of Mines and Mineral Resources, New Mexico Geology, v. 6, no. 1, p. 6-10.

Campbell, F.W., and Roybal, G.H., 1984, Geology and coal resources of the Fence Lake 1:50,000 quadrangle: New Mexico Bureau of Mines and Mineral Resources Open-File Report 207, 37 p.

Hook, S.C., Molenaar, C.M., and Cobban, W.A., 1983, Stratigraphy and revision of nomenclature of Upper Cenomanian to Turonian (Upper Cretaceous) rocks of west-central New Mexico: New Mexico Bureau of Mines and Mineral Resources Circular 185, p. 7-28.

Kelley, V.C., and Northrop, S.A., 1975, Geology of the Sandia Mountains and vicinity, New Mexico: New Mexico Bureau of Mines and Mineral Resources Memoir 29, $136 \mathrm{p}$.

Kottlowski, F.E., Campbell, F.W., Roybal, G.H., and Hatton, K.S., 1984, New Mexico, in 1984 Keystone coal industry manual: New York, McGraw-Hill, p. 558-567.

Landis, E.R., and Dane, C.H., 1969, The Tierra Amarilla coal field, Rio Arriba County, New Mexico: New Mexico Bureau of Mines and Mineral Resources Circular 100, 11 p. 
Landis, E.R., McLellan, M.W., McKay, E.J., Carter, M.D., and Medlin, A.L., 1984, Geologic map of the Fence Lake SW quadrangle, Cibola and Catron Counties, New Mexico: U.S. Geological Survey Miscellaneous Field Studies Map MF-1750, scale 1:24,000.

McLellan, M.W., Biewick, L.R., and Landis, E.R., 1984, Stratigraphic framework, structure, and general geology of the Salt Lake coal field, Cibola and Catron Counties, New Mexico: U.S. Geological Survey Miscellaneous Field Studies Map MF-1689, scale 1:24,000.

McLellan, M.W., Haschke, L.R., and Robinson, L.N., 1982, Geologic map of the Rincon Hondo quadrangle, Cibola County, New Mexico: U.S. Geological Survey Miscellaneous Field Studies Map MF-1506, scale 1:24,000.

McLellan, Marguerite, Haschke, Laura, Robinson, Laura, Carter, M.D., and Medlin, Antoinette, 1983a, Middle Turonian and younger Cretaceous rocks, northern Salt Lake coal field, Cibola and Catron Counties, New Mexico, in Hook, S.C., compiler, Contributions to mid-Cretaceous paleontology and stratigraphy of New Mexico-part II: New Mexico Bureau of Mines and Mineral Resources Circular 185, p. $41-47$.

McLellan, M.W., Haschke, L.R., Robinson, L.N., and Landis, E.R., 1983b, Geologic map of the Moreno Hill quadrangle, Cibola and Catron Counties, New Mexico: U.S. Geological Survey Miscellaneous Field Studies Map MF-1509, scale $1: 24,000$.

McLellan, M.W., Robinson, L.N., and Haschke, L.R., 1983, Geologic map of the Fence Lake quadrangle, Cibola County, New Mexico: U.S. Geological Survey Miscellaneous Field Studies Map MF-1533, scale 1:24,000.

McLellan, M.W., Robinson, L.N., Haschke, Laura, Carter, M.D., and Medlin, A.L., 1982, Fence Lake Formation (Tertiary), west-central New Mexico: New Mexico Geology, v. 4, no. 4 , p. 53-55.

Molenaar, C.M., 1974, Correlation of the Gallup Sandstone and associated formations, Upper Cretaceous, eastern San Juan and Acoma basins, New Mexico: New Mexico Geological Society Guidebook, 25th Field Conference, p. 251-258.
Osburn, J.C., 1982, Geology and coal resources of three quadrangles in the central Datil Mountains coal field, Socorro County, New Mexico: New Mexico Bureau of Mines and Mineral Resources Open-File Report 164, 82 p., $6 \mathrm{pl}$.

1983, Coal resources of Socorro County, New Mexico, in Chapin, C.E., ed., Socorro region II: New Mexico Geological Society Guidebook, 34th Field Conference, 1983, p. 223-226.

1984, Geology of Pueblo Viejo Mesa quadrangle, Socorro and Cibola Counties, New Mexico: New Mexico Bureau of Mines and Mineral Resources Geologic Map 55, scale $1: 24,000$.

Read, C.B., Duffner, R., Wood, G.H., Jr., and Zapp, A.D., 1950, Coal resources of New Mexico: U.S. Geological Survey Circular 89, 24 p.

Roybal, G.H., 1982, Geology and coal resources of Tejana Mesa quadrangle: New Mexico Bureau of Mines and Mineral Resources Open-File Report 178, 38 p.

Roybal, G.H., and Campbell, F.W., 1981, Stratigraphic sequence in drilling data from Fence Lake area, New Mexico: New Mexico Bureau of Mines and Mineral Resources Open-File Report 145, $59 \mathrm{p}$.

Shomaker, J.W., Beaumont, E.C., and Kottlowski, F.E., eds., 1971, Strippable low-sulfur coal resources of the San Juan basin in New Mexico and Colorado: New Mexico Bureau of Mines and Mineral Resources Memoir 25, $189 \mathrm{p}$.

Tabet, D.E., 1979a, Geology of Jornada del Muerto coal field, Socorro County, New Mexico: New Mexico Bureau of Mines and Mineral Resources Circular 168, 19 p.

1979b, Summary of the geology of the Engle coal field: New Mexico Bureau of Mines and Mineral Resources Open-File Report 115, 9 p.

Tabet, D.E., and Frost, S.J., 1978, Coal fields and mines of New Mexico: New Mexico Bureau of Mines and Mineral Resources Resource Map 10.

Winchester, D.E., 1921, Geology of Alamosa Creek valley, Socorro County, New Mexico: U.S. Geological Survey Bulletin 716-A, 15 p. 


\section{SELECTED SERIES OF U.S. GEOLOGICAL SURVEY PUBLICATIONS}

\section{Periodicals}

Earthquakes \& Volcanoes (issued bimonthly).

Preliminary Determination of Epicenters (issued monthly).

\section{Technical Books and Reports}

Professional Papers are mainly comprehensive scientific reports of wide and lasting interest and importance to professional scientists and engineers. Included are reports on the results of resource studies and of topographic, hydrologic, and geologic investigations. They also include collections of related papers addressing different aspects of a single scientific topic.

Bulletins contain significant data and interpretations that are of lasting scientific interest but are generally more limited in scope or geographic coverage than Professional Papers. They include the results of resource studies and of geologic and topographic investigations; as well as collections of short papers related to a specific topic.

Water-Supply Papers are comprehensive reports that present significant interpretive results of hydrologic investigations of wide interest to professional geologists, hydrologists, and engineers. The series covers investigations in all phases of hydrology, including hydrogeology, availability of water, quality of water, and use of water.

Circulars present administrative information or important scientific information of wide popular interest in a format designed for distribution at no cost to the public. Information is usually of short-term interest.

Water-Resources Investigations Reports are papers of an interpretive nature made available to the public outside the formal USGS publications series. Copies are reproduced on request unlike formal USGS publications, and they are also available for public inspection at depositories indicated in USGS catalogs.

Open-File Reports include unpublished manuscript reports, maps, and other material that are made available for public consultation at depositories. They are a nonpermanent form of publication that may be cited in other publications as sources of information.

\section{Maps}

Geologic Quadrangle Maps are multicolor geologic maps on topographic bases in 7 1/2- or 15-minute quadrangle formats (scales mainly $1: 24,000$ or $1: 62,500$ ) showing bedrock, surficial, or engineering geology. Maps generally include brief texts; some maps include structure and columnar sections only.

Geophysical Investigations Maps are on topographic or planimetric bases at various scales; they show results of surveys using geophysical techniques, such as gravity, magnetic, seismic, or radioactivity, which reflect subsurface structures that are of economic or geologic significance. Many maps include correlations with the geology.

Miscellaneous Investigations Series Maps are on planimetric or topographic bases of regular and irregular areas at various scales; they present a wide variety of format and subject matter. The series also includes 7 1/2-minute quadrangle photogeologic maps on planimetric bases which show geology as interpreted from aerial photographs. Series also includes maps of Mars and the Moon.
Coal Investigations Maps are geologic maps on topographic or planimetric bases at various scales showing bedrock or surficial geology, stratigraphy, and structural relations in certain coal-resource areas.

Oil and Gas Investigations Charts show stratigraphic information for certain oil and gas fields and other areas having petroleum potential.

Miscellaneous Field Studies Maps are multicolor or black-andwhite maps on topographic or planimetric bases on quadrangle or irregular areas at various scales. Pre-1971 maps show bedrock geology in relation to specific mining or mineral-deposit problems; post-1971 maps are primarily black-and-white maps on various subjects such as environmental studies or wildemess mineral investigations.

Hydrologic Investigations Atlases are multicolored or black-andwhite maps on topographic or planimetric bases presenting a wide range of geohydrologic data of both regular and irregular areas; principal scale is $1: 24,000$ and regional studies are at $1: 250,000$ scale or smaller.

\section{Catalogs}

Permanent catalogs, as well as some others, giving comprehensive listings of U.S. Geological Survey publications are available under the conditions indicated below from the U.S. Geological Survey, Books and Open-File Reports Section, Federal Center, Box 25425, Denver, CO 80225. (See latest Price and Availability List.)

"Publications of the Geological Survey, 1879-1961" may be purchased by mail and over the counter in paperback book form and as a set of microfiche.

"Publications of the Geological Survey, 1962-1970" may be purchased by mail and over the counter in paperback book form and as a set of microfiche.

"Publications of the U.S. Geological Survey, 1971- 1981" may be purchased by mail and over the counter in paperback book form (two volumes, publications listing and index) and as a set of microfiche.

Supplements for $1982,1983,1984,1985,1986$, and for subsequent years since the last permanent catalog may be purchased by mail and over the counter in paperback book form.

State catalogs, "List of U.S. Geological Survey Geologic and Water-Supply Reports and Maps For (State)," may be purchased by mail and over the counter in paperback booklet form only

"Price and Availability List of U.S. Geological Survey Publicatlons," issued annually, is available free of charge in paperback booklet form only.

Selected coples of a monthly catalog "New Publications of the U.S. Geological Survey" available free of charge by mail or may be obtained over the counter in paperback booklet form only. Thnse wishing a free subscription to the monthly catalog "New Publications of the U.S. Geological Survey" should write to the U.S. Geological Survey, 582 National Center, Reston, VA 22092.

Note.--Prices of Government publications listed in older catalogs, announcements, and publications may be incorrect. Therefore, the prices charged may differ from the prices in catalogs, announcements, and publications. 
